\title{
Acknowledgment to Reviewers of Remote Sensing in 2021
}

\section{Remote Sensing Editorial Office}

MDPI AG, St. Alban-Anlage 66, 4052 Basel, Switzerland

Rigorous peer-reviews are the basis of high-quality academic publishing. Thanks to the great efforts of our reviewers, Remote Sensing was able to maintain its standards for the high quality of its published papers. Thanks to the contribution of our reviewers, in 2021, the median time to first decision was 18 days and the median time to publication was 43 days. The editors would like to extend their gratitude and recognition to the following reviewers for their precious time and dedication, regardless of whether the papers they reviewed were finally published:

A, Geruo

Aakanksha, Rana

Aaron, David

Abarca Del Rio, Rodrigo

Abaspur Kazerouni, Iman

Abate, Dante

Abbas, Azhar

Abbas, Farhat

Abbas, Sawaid

Abbate, Andrea

Abdalla, Rifaat

Abdel-Hamid, Ayman

Abdelkader, Eslam Mohammed

Abdelkareem, Mohamed

Abdelmohsen, Karem

Abdel-Nasser, Mohamed

Abd-Elrahman, Amr

Abdi, Abdulhakim M.

Abdi, Ghasem

Abdi, Omid

Abdollahnejad, Azadeh

Abdolmaleki, Mehdi

Abdullah, Haidi

Abdulridha, Jaafar

Abe, Keita

Abel, Markus

Abera, Temesgen Alemayehu

Aberra, Dawit

Abidin, Hasanuddin Z.

Abood, Sinan

Abotalib, Abotalib Z.

Abramov, Andrey

Abramowski, Tomasz

Abrams, Michael J.
Abrehdary, Majid

Abshaev, Ali

Abubakar, Ismaila Rimi

Abuzar, Mohammad

Abu-Zeid, Nasser

Acción Montes, Álvaro

Acevedo, Rogelio

Acharya, Ram Prasad

Acharya, Tri Dev

Acker, James

Adamczyk, Joanna

Adami, Marcos

Adamo, Maria

Adamopoulos, Efstathios

Adão, Telmo

Adcock, Christopher

Addabbo, Pia

Addamo, Anna M.

Adeli, Solmaz

Adeline, Karine

Adeniyi, Odunayo David

Adesipo, Adegbite

Adhitya, Yudhi

Adinolfi, Guido Maria

Adiri, Zakaria

Adirosi, Elisa

Adler, Petra

Adler-Golden, Steven

Adnan, Farooq

Adnan, Sarfaraz

Adriaensen, Stefan

Aedla, Raju

Afrasinei, Gabriela

Afshar, Mahdi Hesami 
Afshar-Mohajer, Nima

Afzaal, Hassan

Afzali Gorooh, Vesta

Aga, Alemu O.

Agapiou, Athos

Agarwal, Vivek

Agathangelidis, Ilias

Agathos, Alexander

Agegnehu, Sayeh Kassaw

Agrafiotis, Panagiotis

Agrela, Francisco

Agrillo, Emiliano

Agüera-Vega, Francisco

Aguilar, Manuel Ángel

Aguilar-González, Abiel

Aguilera, Cristhian

Aguilera, Felipe

Aguilos, Maricar

Ahamed, Tofael

Ahl, Robert

Ahmad, Mirza Junaid

Ahmad, Muhammad

Ahmad, Touqeer

Ahmad, Uzair

Ahmad, Wael Abdullah

Ahmadi, Ali

Ahmed, Ammar

Ahmed, Farzana

Ahmed, Mohamed

Ahn, Ho-Yong

Ahn, Jae-Hyun

Ahn, Myoung Hwan

Ahokas, E.

Ahrns, Ingo

Ai, Bin

Ai, Jiaqiu

Aiazzi, Bruno

Aichi, Masaatsu

Aichinger-Rosenberger, Matthias

Aida, Kentaro

Aiello, Giuseppe

Aimaiti, Yusupujiang

Aina, Yusuf

AI-Najjar, Husam Abdulrasool H.

Aizenberg, Igor

Ajaz, Ali

Akagic, Amila

Akhtar, Fazlullah

Akmalov, Shamshodbek

Akoğlu, Goze

Akumaga, Uvirkaa
Al Homssi, Bassel

Al Khalifa, Fay

Al Rahhal, Mohamad

Al Sayah, Mario

Alados, Concepcion

Alaggio, Rocco

Alahmadi, Mohammed

Alain, Royer

Alam, Sarfaraz

Alanis, Alma Y.

Alatza, Stavroula

Albano, Christine M.

Alber, Merryl

Alberello, Alberto

Alberton, Bruna

Albizu, Igor

Albizua, Lourdes

Albuquerque, Daniel Filipe

Al-Dousari, Ali

Aldrich, Stephen

Aleinov, Igor

Alejo Eleuterio, Roberto

Alekseeva, Tatiana

Alessandra, Saponieri

Alevizos, Evangelos

Alexakis, Dimitrios D.

Alexan, Wassim

Alexander, Smirnov

Alexandridis, Thomas

Alexandru, Onaca

Al-Farraj, Asma

Alfeld, Matthias

Alfieri, Joseph G.

Alfio, Vincenzo Saverio

Alganci, Ugur

Alger, Matthew J.

Alghamdi, Ali

AlHichri, Haikel

Ali Ahmed, Awadelrahman Mohamedelsadig

Ali, Md. Arfan

Ali, Tarig

Alibakhshikenari, Mohammad

Alicandro, Maria

Alidoost, Fatemeh

Alifu, Haireti

Alissa, Samieh

Alivernini, Alessandro

Alizadeh, Babak

Al-Khaldi, Mohammad M.

Alkhatib, Mohammed

Allabakash, Shaik 
Allahdadi, Mohammad Nabi

Allan, Blake M.

Allan, Craig

Allasia, Paolo

Alleaume, Samuel

Allegra, Dario

Allemand, Pascal

Allen, Michael A.

Allende, Cristina

Al-Madhhachi, Abdul-Sahib

Almagbile, Ali

Almalki, Khalid A.

Almeida, André Quintão

Almeida, Emerson Rodrigo

Almeida, Fernando

Almeida, Marcos

Almeida, Thomas

Almonaci-Caballer, Jaime

Al-Murib, Muhanned D.

Al-Naji, Ali

Al-Najjar, Husam

Al-Nasrawi, Ali K. M.

Aloisi, Alessandra

Alongi, Daniel M.

Alonso Del Rosario, José J.

Alonso, Jose Juan

Alonso-Benito, Alfonso

Alonzo, Michael

Alory, Gaël

Al-Qadi, Imad L.

Al-Quraishi, Ayad M. Fadhil

Alqurashi, Abdullah

Alsalam, Bilal H.

Alshazly, Hammam

Alsina, MariadelMar

Alston, E. J.

Alsweiss, Suleiman

Al-Tahir, Raid

Altamirano-Robles, Leopoldo

Altan, Orhan

Altaratz, Orit

Altdorff, Daniel

Alvarez Mendoza, Cesar Ivan

Álvarez, David González

Alves Ribeiro, Joana

Alves, Tiago M.

Al-Yaari, Amen

Alzubaidi, Laith

Amani, Meisam

Amankwah, Anthony

Amantov, Aleksey
Amaral, Cibele Hummel Do

Amaro-Mellado, José L.

Amato, Umberto

Ambinakudige, Shrinidhi

Ambrosanio, Michele

Ambrožič, Tomaž

Ameen, Bikhtiyar

Amezquita-Semprun, Kendrick

Amici, Andrea

Amigo, José-Manuel

Amin, Ullah

Amini, Amin

Amiridis, Vassilis

Amirkhiz, Reza Goljani

Ammirati, Lorenzo

Amor, Margarita

Amović, Mladen

Ampatzidis, Dimitrios

An, Jiachun

An, Kang

An, Lu

Anagnostopoulos, Georgios

Anand, Anupam

Anand, Prashant

Anantrasirichai, Nantheera

Anastasiou, Evangelos

Anastassopoulos, Vassilis

Ancin-Murguzur, Francisco Javier

Anco, Daniel J.

Anderegg, Jonas

Anderlini, Letizia

Andersen, Brannon

Andersen, Torben E.

Anderson, Carlton P.

Anderson, Dylan

Anderson, Eric

Anderson, Ray

Anderson, Sharolyn

Andersson, Magnus

Ando, Yoshiaki

Andrefouet, Serge

Andrei, Constantin Octavian

Andreić, Željko

Andreo, Veronica

Andrey Andreevich, Golovan

Andries, Ana

Andriolo, Umberto

Andriukaitis, Darius

Andriyanov, Nikita

Andwersen, Ole B.

Aneece, Itiya P. 
Anelli, Debora

Anfuso, Giorgio

Angeletti, Lorenzo

Angelini, Federico

Angelopoulou, Theodora

Angelou, Nikolas

Anguelova, Magdalena D.

Anguilano, Lorna

Anigacz, Wojciech

Annala, Leevi

Annane, Bachir

Annis, Antonio

Anochi, Juliana Aparecida

Ansari, Kutubuddin

Anthes, Richard

Anthony, Stephen

Antón, Daniel

Anton, Iulia-Alina

Antón, Manuel

Antonescu, Bogdan

Antonielli, Benedetta

Antonio, Ricchi

Antonucci, Francesca

Antova, Gergana

Antucheviciene, Jurgita

Anwar, Saeed

Anzidei, Marco

Aoki, Yosuke

Aparicio-Navarro, Francisco J.

Apke, Jason M.

Apollonio, Ciro

Apolo-Apolo, Orly Enrique

Apostol, Bogdan

Appel, Marius

Appeltans, Simon

Appendini, Christian

Ara, Iffat

Aragon, Bruno

Araki, Susumu

Aram, Farshid

Arandjelovic, Ognjen

Arango Galván, Claudia

Aranha, Jose

Arastounia, Mostafa

Araujo, Helder

Arazuri, Silvia

Arbelo, Manuel

Arcos González, Pedro

Ardelean, Florina

Ardila, Juan Pablo

Arena, Fabio
Arenas-Castro, Salvador

Aretano, Roberta

Arganaraz, Juan Pablo

Argenziano, Pasquale

Argiriou, Athanassios A.

Argolo Dos Santos, Robson

Argüello, Francisco

Arias Ballesteros, Manuel

Arias, Luis

Arienzo, Alberto

Aristovnik, Aleksander

Ariza, Alexander

Ariza-Lopez, Francisco Javier

Armadillo, Egidio

Armand, Albergel

Armstrong, Paul

Armstrong, Roy A.

Arnal Barbedo, Jayme Garcia

Arnaubec, Aurélien

Arnold, Andreas

Arnoso, Jose

Arques-Orobon, Francisco Jose

Arranz-Justel, Jose Juan

Arribas, Javier

Arriga, Nicola

Arrouays, Dominique

Arroyo-Mora, J. Pablo

Arseni, Maxim

Artese, Giuseppe

Arteta, Alberto

Artiemjew, Piotr

Aruga, Kazuhiro

Arulraj, Malarvizhi

Arvanitis, Gerasimos

Aryal, Anil

Aryal, Jagannath

Aryal, Yog

Arza, Marcos

Arza-García, Marcos

Asabere, Stephen

Asaka, Tomohito

Asăndulesei, Andrei

Asanuma, Ichio

Aschonitis, Vassilis George

Asgarimehr, Milad

Ash, Konstantin

Ashapure, Akash

Ashby, Jeffrey

Ashfaq Khan, Muhammad

Ashraf, Fahmidah U.

Aslan, Gökhan 
Asmaryan, Sh G.

Asong, Zilefac Elvis

Asplin, Matthew

Assireu, Arcilan

Assumma, Vanessa

Astitha, Marina

Atanasoae, Pavel

Atherton, Jon

Athreyas, Kashyap Naren

Atique, Usman

Atkins, Jeff

Atkinson, Alan D.

Atkinson, Nigel

Atoche, Alejandro Castillo

Attila, Fazekas

Auad, Guillermo

Aubert, Hervé

Auch, Roger

Aufaristama, Muhammad

Auger, Jeffrey D.

Augustin, Patrick

Aulicino, Giuseppe

Aun, Margit

Aung, Zeyar

Autrey, Bradley

Avalos, Gerardo

Ávalos, Juan Gerardo

Avdellidou, Chrysa

Avila, Alvaro Javier

Avila, Sergio Luciano

Avino, Pasquale

Avola, Giovanni

Avolio, Corrado

Avolio, Elenio

Avotniece, Zanita

Avouris, Dulcinea

Awad, Mohamad

Ayala, David

Ayala-Cabrera, David

Ayhan, Bulent

Ayoub, Naeem

Azadi, Hossein

Azamt, Muhammad

Azañón Hernández, Jose Miguel

Azar, Ramin

Azarang, Arian

Azeiteiro, Ulisses Miranda

Azevedo, Fábio

Azimi, Mohsen

Azimjon, Sayidov

Aziz, Ammar Abdul
Aziz, Ballouche

Azman, Lucija

B. D., Parameshachari

Bâ, Khalidou M.

Baade, Jussi

Baars, Holger

Baba, Mohamed Wassim

Babcock, Esther L.

Babichev, Sergii

Babu, Arun

Babu, S. Ravindra

Bacanin, Nebojsa

Bachhofner, Stefan

Bachman, Scott D.

Bachmann, Martin

Bacic, Zeljko

Backoulou, Georges F.

Ba,czyk, Marcin Kamil

Badarau, Alexandru Sabin

Badea, Ana Cornelia

Badea, Ovidiu

Badescu, Gabriel

Badger, Merete

Badreldin, Nasem

Badulin, Sergei I.

Badura, Gregory P.

Bae, Gwi Nam

Bagaglini, Leonardo

Baganha Baptista, Paulo Renato

Baghdadi, Nicolas N.

Bagli, Stefano

Bahamon, Nixon

Bai, Geng

Bai, Peng

Bai, Tao

Bai, Weihua

Bai, Xiaojing

Bai, Xiwei

Baier, Bianca

Bailey, Donald

Bainbridge, Scott

Baiocchi, Valerio

Bajić, Dragoljub

Bak, Juseon

Bakator, Mihalj

Baker, Robert L.

Bakhmetieva, Nataliya V.

Bakker, Wim

Bakuła, Mieczysław

Balado Frias, Jesus

Balaguru, Karthik 
Balal, Nezah

Balasubramani, Booma Sowkarthiga

Balasubramanian, Sundarabalan

Balawejder, Monika

Balázs, Varga

Bałazy, Radomir

Balbarani, Sebastián

Balčiauskas, Linas

Balcik, Filiz Bektas

Baldini, Luca

Bałdysz, Zofia

Balenović, Ivan

Baležentienè, Ligita

Balicki, Jerzy

Balidakis, Kyriakos

Ballabrera Poy, Joaquim

Ballari, Daniela

Ballarotta, Maxime

Ballesteros, Dora M.

Ballinger, Tom

Balme, Matthew

Baloch, Zafar

Balsa, José

Balsa-Barreiro, Jose

Balsi, Marco

Balss, Ulrich

Baltas, Evangelos

Balz, Timo

Bambach, Nicolas

Banan, Zoya

Banaszuk, Piotr

Bandara, Ranmalee

Bandera, Antonio

Banerjee, Bikram

Banerjee, Ruman

Banic, Milan

Bank, Carl-Georg

Banks, Andrew Clive

Bannari, Abderrazak

Bannister, Ross

Bao, Fangwen

Bao, Lifeng

Bao, Nisha

Baptista, Mariana D.

Baradello, Luca

Baraka, Moustafa

Baran, Perver

Baray, Jean Luc

Barb, Adrian S.

Barbano, Francesco

Barbati, Maria
Barbetta, Silvia

Barbetti, Roberto

Barbon Junior, Sylvio

Barbosa, B. D. S.

Barbosa, Henrique De Melo Jorge

Barbosa, Humberto

Barbu, Nicu

Barbulescu, Alina

Barca, Emanuele

Barcik, Peter

Barczyk, Martin

Barentine, John

Bari, Driss

Barisic, Ivana

Barjaktarović, Marko Č.

Barka, Ivan

Barker, Jason

Barker, Michael Kenneth

Barlyaeva, Tatiana

Barnes, Robert

Barnoaiea, Ionut

Barone, Pier Matteo

Barr, Jordan G.

Barrena-González, Jesús

Barrett, Alexander

Barrios, José Miguel

Barrios, Miguel

Barrios, Yubal

Barriot, Jean-Pierre

Barros, Pedro Paulo Silva

Barsanti, Gonizzi

Barsanti, Mattia

Barsanti, Michele

Barsanti, Sara Gonizzi

Bartlett, Paul

Bartlow, Andrew W.

Barton, Craig

Barton, Eric Desmond

Barton, Iván

Bartrina, Joan

Barz, Cristian

Barzaghi, Stefania

Basbas, Socrates

Baselt, Ivo

Basford, Philip J.

Basha, Elizabeth

Bashar, Syed Khairul

Bashkuev, Yu B.

Bashmachnikov, Igor L.

Bašić, Tomislav

Basil, Christaras 
Basilici, Matteo

Bassan, Massimo

Bassani, Cristiana

Bastarrika, Aitor

Basterrechea Chertudi, Daniel

Basuki, Tyas Mutiara

Batchuluun, Ganbayar

Bathrellos, George

Batista, Celene

Battaglia, Alessandro

Battaglia, Maurizio

Battiston, Roberto

Baudry, Jacques

Bauer, Miroslav

Bauer, Ralf

Bauer, Sven

Bauer, Thomas

Baugh, Kim

Baumann, Sabine

Bavle, Hriday

Bawazir, A. Salim

Bayarri, Vicente

Bayat, Nozhan

Bazan, Giuseppe

Bazanowski, Maciej

Bažec, Matej

Bazi, Yakoub

Bazighifan, Omar

Bažok, Renata

Bazzaz, Mohammad

Bazzi, Hassan

Bazzino Ferreri, Gaston A.

Beach, Robert

Beamish, Alison

Beard, Kate

Beben, Damian

Beć, Krzysztof B.

Becek, Kazimierz

Becerra, Miguel A.

Becerril, Hilario

Bech, Joan

Bechtel, Timothy D.

Becker, Marcelo

Becker, Rolf

Becker-Reshef, Inbal

Beckers, Joost

Bȩdkowski, Janusz Marian

Bednorz, Ewa

Beerens, Peter

Beeri, Ofer

Beeson, Peter C.
Begeman, Carolyn Branecky

Beggs, Helen

Begin, Daniel

Begue, Nelson

Behera, Mukund

Behling, Robert

Bejan, Iustinian

Bekova, Radoslava

Belanovic Simic, Snezana

Belart, Joaquin

Belfiore, Oscar Rosario

Belikov, Dmitry

Bell, David

Bell, Jordan R.

Bellacicco, Marco

Bellasio, Roberto

Bellman, Chris

Bellocchio, Enrico

Bellucci, Giancarlo

Belmonte Fernández, Oscar

Belward, Alan

Benali, Akli

Benassai, Guido

Bender, Michael

Bendini, Hugo Do Nascimento

Bendor, Eyal

Benedetti, Elisa

Benedict, Karl

Benhalouche, Fatima Zohra

Beninde, Joscha

Benitez-Restrepo, Hernan Dario

Benjamin, Adam

Benmergui, Joshua

Bennett, Mia M.

Bentel, Katrin

Bentes, Carlos

Bento-Gonçalves, António

Berg, Aaron

Bergado, John

Bergamasco, Filippo

Berger, Katja

Berglund, Robin

Bergsma, Erwin

Berline, Leo

Bernacki, Bruce

Bernardo, Nariane

Bernhard, Luzi

Bernier, Monique

Berra, Elias Fernando

Bertacchi, Andrea

Bertalan, László 
Bertocci, Francesco

Bertocci, Stefano

Bertoldi, Giacomo

Bertolo, Andrea

Bertolo, Davide

Bertuch, Thomas

Besse Rimba, Andi

Besson, Olivier

Bettencourt, João Paulo

Bettenhausen, Michael H.

Betz, Florian

Beu, Dorin

Beuth, Frederik

Bevan, Elizabeth

Beyer, Hans Georg

Beylich, Achim

Bezdan, Atila

Bhadra, Sourav

Bhandari, Santosh

Bhanja, Soumendra

Bhargava, Kriti

Bhat, Deepak R.

Bhattacharjee, Shrutilipi

Bhattacharya, Devanjan

Bhattarai, Keshav

Bhimireddy, Sudheer R.

Bhowmick, Sutanu

Bhuiyan, Ehsan

Bhuiyan, Md Abul Ehsan

Bhuyian, Md.

Bi, Haixia

Bi, Jian

Bi, Shun

Biagi, Pier Francesco

Bialek, Aga

Bialik, Robert

Bian, Liheng

Bian, Xiaolin

Bian, Zunjian

Biancolillo, Alessandra

Biasotti, Silvia

Bickel, Valentin

Bickel, Valentin Tertius

Bidel, L. P. R.

Bieda, Agnieszka

Biegun, Dariusz

Biggs, Trent

Biglia, Alessandro

Bigman, Daniel

Bignami, Christian

Bijak, Szymon
Bilal, Muhammas

Bilasco, Stefan

Bilham, Roger

Billah, Mirza

Bilotta, Giuliana

Bilous, Andrii

Binding, Caren

Bindzárová Gergelová, Marcela

Bingham, Frederick

Binyamin, Jacqueline

Binzhong, Zhou

Biondi, Filippo

Birk, Andreas

Birylo, Monika

Biscaro, Thiago S.

Bisci, Carlo

Bishop, Michael P.

Bishop, Tara B. B.

Biswas, Nishan Kumar

Biswas, Sounak

Bitencourt, Marisa

Bithas, Petros

Biudes, Marcelo Sacardi

Bizjak, Marko

Bizzarri, Roberto

Bjelopera, Anamaria

Björkman, Mårten

Black, Robert

Blagojević, Aleksandar

Blagoveshchenskaya, Natalia F.

Blahut, Jan

Blake, David

Blanco, Juan A.

Blanco-Sacristán, Javier

Blanes, Ian

Blankenbach, Jörg

Błaszczak-Bąk, Wioleta

Blesic, Suzana

Blesius, Leonhard

Blevins, Robert

Bliss, Andrew

Blištan, Peter

Blonski, Slawomir

Bluhm, Richard

Blumler, Mark

Bo, Yanchen

Bobkowska, Katarzyna

Bobulska, Lenka

Boccia, Lorenzo

Boccolari, Mauro

Bochenek, Zbigniew 
Bočić, Neven

Bodanese, Eliane

Bode, Robert

Bodian, Ansoumana

Bogachev, Mikhail

Bogawski, Paweł

Bogdan, Grzegorz

Bogdan, Ileana

Bogdan, Mihai

Bogdan, Ruszczak

Bogdanov, Sergey

Bohak, Ciril

Bohm, Emanuele

Böhm, Johannes

Bojarova, Jelena

Boldrini, Enrico

Boldyreff, Anton S.

Bolfe, Édson Luis

Bolkas, Dimitrios

Bolla, Alberto

Bolliger, Ian

Bolognesi, Cecilia Maria

Bolognesi, Salvatore Falanga

Boltižiar, Martin

Bolton, Douglas

Bonafoni, Stefania

Bonali, Fabio Luca

Bonasia, Rosanna

Bond, Jason

Bonduà, Stefano

Bonenberg, Lukasz

Bonì, Roberta

Boniface, Karen

Bonin, Jennifer

Bonnet, Pierre

Bonora, Valentina

Boodoo, Sudesh

Booth-Rea, Guillermo

Boothroyd, Richard J.

Bopape, Mary-Jane Morongwa

Borchevkina, Olga

Bordogna, Gloria

Bordoni, Federica

Bordoni, Massimiliano

Boreggio, Mauro

Borfecchia, Flavio

Borge Holthoefer, Javier

Borges, Díbio Leandro

Borges, José Fernando

Borghi, Alessandra

Borisov, Mirko
Borja, Maria Olga

Borkowski, Piotr

Borma, Laura De Simone

Boros-Lajszner, Edyta

Borowska-Stefańska, Marta

Borowski, Łukasz

Borrelli, Luigi

Borruso, Giuseppe

Bortoli, Daniele

Borusiewicz, Andrzej

Borz, Stelian Alexandru

Borzì, Iolanda

Bos, Machiel

Bosch, Juan Luis

Boschetti, Luigi

Bosch-Lluis, Xavier

Bosino, Alberto

Bosisio, Ada Vittoria

Bosnic, Ivana

Bosso, Luciano

Bostater, Charles R.

Botezan, Camelia S.

Botha, Elizabeth

Botzheim, Janos

Bouali, El Hachemi

Boulet, Gilles

Bourassa, Mark

Bourgeois, Jean

Bourlier, Christophe

Boutsi, Argyro-Maria

Bouvier, Clément

Bouzinac, Catherine

Bovenga, Fabio

Boy, Jean-Paul

Boyd, Bill

Boyd, Dylan Ray

Božinović, Sofija

Bozzano, Francesca

Braaten, David

Brach, Michał

Brack, Andreas

Bradford, Lori

Bramley, Rob

Brancato, Virginia

Brandão, Vasco André Barbosa

Brandes, Christian

Brandini, Carlo

Brandolini, Filippo

Brandon, Christine

Brands, Edwin

Brandt, Carlos Henrique 
Brandt, Peter

Brandusoiu, Ionut

Brankov, Milan

Bräuer-Burchardt, Christian

Braun, Alexander

Braunmiller, Jochen

Brazel, Anthony

Brčić, David

Brede, Benjamin

Bren, Leon

Bréon, François-Marie

Bresciani, Mariano

Bretcan, Petre

Bretfeld, Mario

Brezzi, Lorenzo

Bridges, John

Brigante, Raffaella

Briganti, Riccardo

Bright, Benjamin C.

Brimblecombe, Peter

Bringer, Alexandra

Brinkhoff, James

Brinkworth, Russell

Brisset, Elodie

Brivio, Pietro A.

Briz-Redón, Álvaro

Broadbent, Eben N.

Brocca, Luca

Brockmann, Carsten

Brodny, Jarosław

Brolly, Gábor

Brolly, Matthew

Buler, Jeffrey

Bulgariu, Laura

Bullock, Eric

Bulusu, Subrahmanyam

Bundzel, Marek

Bunn, Michael

Bunting, Erin

Buongiorno, Fabrizia

Buono, Andrea

Burai, Péter

Burcea, Sorin

Burdun, Iuliia

Burdziakowski, Paweł

Burgdorf, Martin

Burget, Radim

Burghardt, Andrzej

Burian, Adina

Burlakovs, Juris

Burnette, Dorian J.
Burns, Patrick

Burr, Ralf

Burrows, Katy

Burt, Peter

Bury, Grzegorz

Bus, Agnieszka

Bushueva, Irina

Busico, Gianluigi

Buttafuoco, Gabriele

Butts-Wilmsmeyer, Carolyn

Bystrov, Alex

Cabaleiro, Manuel

Cabezas, José

Cabos, William

Cabral, Ana I. R.

Cabral, Pedro

Cabrera, Francisco

Cabrera, Humberto

Cabrera-Revuelta, Elena

Cabrillo, Raquel Somavilla

Caccavale, Mauro

Cachorro, Victoria E.

Cadeddu, Maria

Cadol, Daniel

Cafaro, Francesco

Cafolla, Daniele

Cai, Liping

Cai, Wenyu

Cai, Xuguang

Cai, Yaping

Cao, Siyang

Cao, Xin

Cao, $\mathrm{Yu}$

Cao, Yue-Ling

Cao, Zhigang

Caparrini, Francesca

Capelli, Achille

Capineri, Lorenzo

Capizzi, Patrizia

Capobianco, Giuseppe

Capolupo, Alessandra

Caporali, Alessandro

Capozzi, Vincenzo

Cappadonia, Chiara

Capra, Alessandro

Capra, Gian Franco

Capraro, Flavio

Caputo, Teresa

Caraballo, Ramon

Caramihai, Simona Iuliana

Carbonell Carrera, Carlos 
Cardellach, Estel

Cardello, Giovanni Luca

Cardillo, Emanuele

Cardim, Guilherme Pina

Cardona-morales, Oscar

Cardone, Daniela

Carfi, Salvatore

Caridade, Pedro Jorge Dos Santos Branco

Cariou, Claude

Carli, Cristian

Carlino, Stefano

Carlo, Bianchini

Carlson, Barbara E.

Carlson, Toby

Carlström, Anders

Carminati, Fabien

Carmona, Emiliano

Carmona, Pedro Latorre

Carmona-Moreno, César

Carneiro, Eduilson

Caro-Cuenca, Miguel

Carr, Rachel

Carranza-García, Manuel

Carrasco Tornero, Luis

Carrasco, Ana Rita

Carrasquero-Durán, Armando

Carraz, Olivier

Castro-Arvizu, Juan Manuel

Castrorao Barba, Angelo

Casula, Giuseppe

Catalan, Patricio

Catalão, João

Catalin, Dumitrescu

Cățeanu, Mihnea

Catita, Cristina

Catry, Filipe

Causa, Flavia

Caussel, Ean-François Mas

Cavalaris, Chris

Cavalieri, Daniel Cruz

Cavalli, Marco

Cavalli, Rosa

Cavalli, Rosa Maria

Caya, Alain

Cazacu, Constantin

Cazacu, Marius Mihai

Cazcarra-Bes, Victor

Cazzaniga, Noemi E.

Ceamanos, Xavier

Cebulska, Marta

Cedro, Anna
Cefalo, Raffaela

Cejnek, Matous

Celesti, Marco

Cen, Yi

Centeno, Jorge Antonio Silva

Ceola, Serena

Cera, Valeria

Cerasuolo, Giovanni

Cernadas, Eva

Cerrillo-Cuenca, Enrique

Cetin, Haluk

Cha, Joowan

Chai, Dengfeng

Chai, Tianfeng

Chai, Young Ho

Chakhar, Amal

Chakraborty, Shamik

Chakraborty, Subrata

Chakraborty, Tirthankar

Chakravarthi, Bharatesh

Chakravarty, Sumit

Chamberlin, Phillip

Chan, Benny Kwok Kan

Chan, Teck Kai

Chen, Changjun

Chen, Chao

Chen, Cheng

Chen, Chen-Tung Arthur

Chen, Chien-yuan

Chen, Chuanfa

Chen, Dingjiang

Chen, Dong

Chen, Erxue

Chen, Fan

Chen, Fulong

Chen, Gang

Chen, Ge

Chen, Hongda

Chen, Hungen

Chen, Huopo

Chen, Jan-Chang

Chen, Jiahong

Chen, Jianlai

Chen, Jiawei

Chen, Jie

Chen, Jifa

Chen, Jizu

Chen, Jorge

Chen, Jun

Chen, Junping

Chen, Kejie 
Chen, Kongzhe

Chen, Lisi

Chen, Maolin

Chen, Mi

Chen, Min

Chen, Mingli

Chen, Peng

Chen, Pengfei

Chen, Qi

Chen, Qusen

Chen, Roufei

Chen, Ruishan

Chen, Ruiyao

Chen, Shangfeng

Chen, Sheng-Po

Chen, Shih-Yu

Chen, Tao

Chen, Tsu-Wei

Chen, Tzu-Ling

Chen, Wei

Chen, Wei-Bo

Chen, Wei-Nai

Chen, Weiwei

Chigbu, Uchendu Eugene

Chikita, Kazuhisa A.

Chintakunta, Harish

Chirico, Peter G.

Chirima, George

Chiriyath, Alex

Chishtie, Farrukh

Chiteculo, Vasco

Chmielewski, Leszek J.

Chmielewski, Szymon

Cho, Jaeil

Cho, Sung Ho

Choe, Yoonsik

Choi, Hyo

Choi, Jaewan

Choi, Jin

Choi, Jong-Kuk

Choi, Min-Kook

Choi, Wonei

Choi, Yeji

Choi, Yosoon

Choo, Youngmin

Chou, Tien-Yin

Choung, Yunjae

Chow, Desmond

Chowdhury, Abu Sayed

Chowdhury, Pranab K. Roy

Christian, Kenneth Edward
Christie, Frazer

Christman, Zachary

Christodoulakis, John

Christof, Lüpkes

Christopher, Ilori

Christopoulou, Anastasia

Chrysafis, Irene

Chu, Hone-Jay

Chu, Lixia

Chu, Ta-Shun

Chua, Ming-Yam

Chuang, Wei-Liang

Chuang, Yung-Chung

Chukin, Vladimir

Chun, Pang-jo

Chung, Kao-Shen

Chung, Wonzoo

Chung, Yong Suk

Chung, Yongwha

Chung, Young-seek

Churnside, James

Chybicki, Andrzej

Cigna, Francesca

Ciracì, Enrico

Cokelet, Edward

Cole, Jason N. S.

Coleman, Richard

Collado-Montero, Francisco José

Colleter, Rozenn

Collins, Michael D.

Collister, Brian

Colom, Miguel

Colombani, Nicolo

Colombatti, Giacomo

Colombo, Claudio

Colosi, Francesca

Colosimo, Gabriele

Colpaert, Alfred

Coluzzi, Rosa

Combes, Didier

Combot, Clément

Comite, Davide

Concha, Javier A.

Conde, FranciscoCarreño

Condé, Tiago $\mathrm{M}$.

Condorelli, Francesca

Conforti, Massimo

Confuorto, Pierluigi

Cong, Wang

Congalton, Russell

Connette, Grant 
Connolly, Harold C., Jr.

Connor, Charles

Constantin, Nistor

Conti, Alessandro

Conti, Livio

Conticello, Federico Rosario

Contini, Daniele

Contreras, Dan

Contreras-López, Manuel

Contreras-Medina, Luis

Conyers, Lawrence B.

Cooley, Sarah

Cooper, Matthew A.

Cooper, Matthew G.

Corazza, Maria Vittoria

Corbari, Chiara

Corbau, Corinne

Corbea-Pérez, Alejandro

Corbella, Ignasi

Corbelle Rico, Eduardo Jose

Corbí, Hugo

Crosby, Michael K.

Crosman, Erik

Cross, Matthew

Crosta, Álvaro

Croteau, Michael

Crow, Wade

Cruvinel, Paulo E.

Cruz, Edmanuel

Cruz, Henry

Csiszar, Ivan

Csomós, György

Csurgai-Horváth, László

Cucci, Davide Antonio

Cuccoli, Fabrizio

Cudahy, Thomas John

Cudlín, Pavel

Cuenca-Garcia, Carmen

Cuevas De La Rosa, Francisco

Cui, Jiashan

Cui, Tengfei

Cui, Xiangbin

Cui, Xihong

Cui, Yi

Cuiñas, Iñigo

Cunha, Ana Paula Martins Do Amaral

Cunha, Fernando França

Cunha, Mario

Curci, Gabriele

Currey, Chris

Cushman, K. C.
Custodio, Danilo

Cusworth, Daniel

Cutter, George

Cvijetinović, Željko

Ćwiąkała, Paweł

Cyril, Palerme

Czaplewski, Krzysztof

Czaplewski, Raymond

Czarnogorska, Magdalena

Czechowski, Leszek

Czerniawski, Thomas

Czuba, Jonathan A.

Czyża, Szymon

D'Amato, Francesco

D'Angelo, Gennaro

D'Auria, Luca

D'Urso, Guido

Da Conceicao Bispo, Polyanna

Da Silva Camargo, Sandro

Dasgupta, Antara

Dash, Prasanjit

Dattola, Luigi

Daum, Fred E.

Dauteuil, Olivier

David, Ciprian

Davidar, Priya

Davidavičienè, Vida

Davids, Corine

Davies, Kevin P.

Davis, Dylan

Davis, Lloyd

Davranche, Aurélie

Dawes, Warrick R.

Dawidowicz, Agnieszka

Dawidowicz, Karol

Dawson, Kyle

Day, Mackenzie

Day, Stephanie

Dayanandan, Baiju

De Abelleyra, Diego

De Aguiar, Claudinei Rodrigues

De Alban, Jose Don T.

De Alckmin, Gustavo Togeiro

De Almeida, Cláudia

De Bem, Pablo Pozzobon

De Carolis, Giacomo

De Carvalho Junior, Osmar Abilio

De Cola, Tomaso

De Costa Trindade Amorim, Margarete Cristiane

De Figueiredo, Tomás

De Francesco, Maria Carla 
De Keukelaere, Liesbeth

De La Casa, Antonio

De La Prieta Pintado, Fernando

De Lima, Aline Maria Meiguins

De Los Reyes, Raquel

De Luca, Davide Luciano

De Macedo, Carina Regina

De Marco, Raffaella

De Martino, Salvatore

De Miguel, Argimiro

De Miguel-Vela, Gonzalo

De Napoli, Luigi

De Oliveira Moraes, Alison

De Oliveira, Aline Macedo

De Oliveira, Julio Cesar

De Pablo, M. A.

De Paula, Eurico Rodrigues D.

De Pinho, Carolina Moutinho Duque

De Sanjosé Blasco, José Juan

De Santis, Angelo

De Smet, Timothy S.

Deb, Proloy

Debaene, Guillaume

DeBeer, Chris

Deborah, Hilda

Declercq, Pierre-Yves

Deelstra, Johannes

DeFelice, Tom

Defraigne, Pascale

Degerickx, Jeroen

Deiana, Giacomo

Deilami, Kaveh

Dejoux, Jean-François

Dekavalla, Maria

Dekemper, Emmanuel

Dekker, Arnold G.

Del Águila, Ana

Del Barrio, Gabriel

Del Core, Giuseppe

Del Hougne, Philipp

Del Pizzo, Silvio

Del Pozo, Susana

Del Río, Veronica Santalla

Del Soldato, Matteo

Delalieux, Stephanie

Delaney, Keith B.

Delapasse, Joao

Delcea, Camelia

Delegido Gómez, Jesús

Delene, David J.

Delepoulle, Samuel
Delgado, Ana Laura

Delgado, Francisco

Delgado, Jorge

Delgado, Rafael

Delgado, Rubén

Delgado-Bonal, Alfonso

Deligiannakis, Georgios

Delipetrev, Blagoj

Dell, Bernard

Dell' Acqua, Fabio

Delle Donne, Dario

Delrieux, Claudio

Demattê, José

Dematteis, Niccolò

Demertzis, Konstantinos

Demetrescu, Emanuel

Demir, Nusret

Demircan, Mesut

Demirel, Mehmet Cüneyd

Demontoux, François

Demyanov, Vladislav

Deng, Chenbin

Deng, Hongbin

Deng, Liang-Jian

Deng, Licai

Deng, Qinghai

Deng, Xiaolin

Deng, Yingbin

Deng, Zhiguo

Denisenko, Valery V.

Déniz, Oscar Deniz

Deniziak, Stanisław

Deo, Manoj

Depina, Ivan

Der Sarkissian, Rita Georges

Derakhshan, Sahar

Derkani, Marzieh H.

Dérobert, Xavier

Deruyter, Greet

Deshmukh, Parik

Dettmering, Denise

Deumlich, Detlef

Deutsch, Ariel N.

Deutscher, Janik

Deutscher, Nicholas M.

Devadiga, Sadashiva

Devine, Scott M.

Devleeschouwer, Xavier

Devoti, Roberto

Devoto, Stefano

DeVries, Ben 
Dewan, Ashraf

Dewi, Christine

Dewi, Ratna Sari

Dewitte, Olivier

Dewitte, Steven

Dey, Sayan

Dhakal, Subodh

Dhariwal, Rohit

Dharmalingam, Selvaraj

Dhawale, Nandkishor M.

Di Benedetto, Alessandro

Di Bernardino, Annalisa

Di Carlo, Fabio

Di Maio, Rosa

Di Martino, Ferdinando

Di Martire, Diego

Di Matteo, Lucio

Di Paola, Francesco

Di Pinto, Valerio

Di Risio, Marcello

Di, Kaichang

Di, Zhenhua

Diaconita, Vlad

Diaconu, Daniel Constantin

Diakogiannis, Foivos I.

Diamanti, Nectaria

Diana, Lorenzo

Diao, Chunyuan

Diara, Filippo

Dias, Andre

Dias, Mauricio

Dias, Tiago

Díaz Delgado, Carlos

Díaz-Morcillo, Alejandro

Díaz-Ruíz, Francisco

Diaz-Sarachaga, Jose Manuel

Dibari, Camilla

Didebulidze, Goderdzi

Diémoz, Henri

Dietrich, Ottfried

Dietz, Andreas J.

Dieudonné, Elsa

Diez, Sebastian

Diez, Yago

Dikshit, Abhirup

Dill, Robert

Dille, Antoine

Dilo, Arta

Dima, Mihai

Dimitriadis, Panayiotis

Dimitrijevic, Aleksandar
Dimou, Anastasios

Dimov, Dimo

Dimuccio, Luca

DiNapoli, Robert

Dinelli, Bianca Maria

Ding, Jiachen

Ding, Kaimeng

Ding, Yang

Ding, Yixing

Dinis, Rui

Diniz, Cesar

Dinoi, Adelaide

Diomede, Tommaso

Dipendra, Gautam

Diraco, Giovanni

Discenza, Marco Emanuele

Disney, Mathias

Disperati, Leonardo

Dissanayake, Dmslb

Dixon, Mike

Djaman, Koffi

Djenouri, Youcef

Djurović, Igor

Dlapa, Pavel

Dmowska, Anna

Dobesova, Zdena

Dobler, Gregory

Dobrev, Nikolai

Dobrinić, Dino

Dobslaw, Henryk

Doelling, Dave

Dogaru, Traian

Doglioni, Angelo

Dohnal, Filip

Doke, Ryosuke

Dokladal, Petr

Doktor, Daniel

Dolan, Brenda

Dolgikh, Grigory Ivanovich

Dolin, Lev S.

Dolinaj, Dragan

Domej, Gisela

Domingo, Dario

Dominguez Gomez, Jose Antonio

Dominici, Donatella

Domitrović, Josipa

Donadio, Carlo

Donati, Davide

Doncov, Nebojsa

Doneus, Michael

Donezar, Uxue 


\begin{tabular}{|c|c|}
\hline Dong, Di & Du, Shouji \\
\hline Dong, Lihu & Du, Yanling \\
\hline Dong, Qinghan & Du, Yongming \\
\hline Dong, Runmin & Du, Yun \\
\hline Dong, Shaochun & Duan, Si-Bo \\
\hline Dong, Sijun & Duan, Weili \\
\hline Dong, Taifeng & Duarte, André \\
\hline Dong, Xichao & Duarte-Carvajalino, Julio M. \\
\hline Dong, Yanni & Dubbini, Marco \\
\hline Dong, Yingying & Dube, Timothy \\
\hline Dong, Zhen & Dubreuil, Vincent \\
\hline Donini, Elena & Dubucq, Dominique \\
\hline Donnini, Marco & Ducati, Jorge Ricardo \\
\hline Dooley, Claire & Duchesne-Onoro, Rocio R. \\
\hline Dordas, Christos A. & Ducke, Benjamin \\
\hline Dornbusch, Uwe & Dudczyk, Janusz \\
\hline Dos Santos Prol, Fabricio & Dudhia, Jimy \\
\hline Dos Santos, Carlos Antonio Costa & Dugdale, Tony M. \\
\hline Dos Santos, Renato César & Dugelay, Samantha \\
\hline Dosso, Paolo & Dugonjić Jovančević, Sanja \\
\hline Dostál, Ivo & Duine, Gert-Jan \\
\hline Dostálová, Alena & Dumić, Emil \\
\hline Dou, Yue & Dumitrescu, Alexandru \\
\hline Doughty, Cheryl & Dumont, Stéphanie \\
\hline Doughty, Rusell & Duncan, Bryan \\
\hline Douglas, Ian & Dunea, Daniel \\
\hline Doulamis, Anastasios & Dungan, Jennifer \\
\hline Doulamis, Nikolaos & Duplančić Leder, Tea \\
\hline Doxaran, David & Duque Cardona, Juan Carlos \\
\hline Draganov, Deyan & Duque-Domingo, Jaime \\
\hline Dragicevic, Arnaud Z. & Durango Cordero, Juan \\
\hline Drago, Carlo & Ďurčanská, Daniela \\
\hline Dragut, Lucian & Durcik, Matej \\
\hline Drap, Pierre & Duta, Alina \\
\hline Drapikowski, Paweł & Dutra, Andeise Cerqueira \\
\hline Dreglea, Aliona & Dvornikov, Yury \\
\hline Dresp-Langley, Birgitta & Dvorsky, Matthew R. \\
\hline Drewnik, Marek & Dwomoh, Francis \\
\hline Drljaca, Miroslav & Dybbroe, Adam \\
\hline Drobnjak, Siniša & Dyer, Jamie \\
\hline Drobot, Radu & Dyke, Shirley J. \\
\hline Dronova, Iryna & Dysarz, Tomasz \\
\hline Druckenmiller, Matthew L. & Dzambo, Andrew \\
\hline Drusa, Marián & Dzikuć, Maciej \\
\hline Drzewiecki, Wojciech & Džunda, Milan \\
\hline Du Plessis, Warren & E Beck, Hylke \\
\hline Du, Huaqiang & Eady, Matthew \\
\hline Du, Jinyang & Eastin, Matthew D. \\
\hline Du, Lan & Ebada, Ahmed Ismail \\
\hline Du, Lin & Ebel, Patrick \\
\hline Du, Mingyuan & Ebinuma, Takuji \\
\hline
\end{tabular}


Edelen, Jonathan

Edemskiy, Ilya K.

Edgington, Duane

Efremova, Boryana

Egaña, Alvaro

Egi, Yunus

Egidarev, Eugene

Egipto, Ricardo

Eguchi, Nawo

Ehmele, Florian

Eichelberger, Hans U.

Eineder, Michael

Einfalt, Thomas

El Amraoui, Laaziz

El Kenawy, Ahmed

El-Alem, Anas

Elangovan, Vinayak

El-Ashmawy, Nagwa

El-Awamry, Ahmed

Elaziz, Mohamed Abd

Elbahnasawy, Magdy

Elbayoumi, Tamer

Elberink, Sander Oude

Eleftheratos, Kostas

Elfadaly, Abdelaziz

Elguennouni, Youssef

Elhabiby, Mohamed

Elhag, Mohamed

Elhattab, Ahmed

El-Hefny, Mervat

El-Hendawy, Salah

Elhoseny, Mohamed

Eliades, Marinos

Eliasson, Jonas

Eliseev, Alexey

Elken, Jüri

Ellenburg, Lee

Ellicott, Evan

Ellman, Artu

Elmahdy, Samy

Elmes, Arthur

Elmouttie, Marc

Elragal, Ahmed

El-Rawy, Mustafa

ElSaadani, Mohamed

Elsaesser, Gregory

Elsaid, Abdelrahman

Elsakov, Vladimir

ElSayed, Ahmed

El-Shafai, Walid

Elsheikh, Mohamed
Elshikha, Diaa Eldin M.

Elżbieta, Bielecka

El-Zeiny, Ahmed

Emami, Ebrahim

Emberson, Robert

Emde, Claudia

Emmer, Adam

Emmitt, Joshua

Enciso, Juan

Ene, Dragos

Enea, Andrei

Eneva, Mariana

Engler, Evelin

Engstrom, Ryan

Enríquez, Carlos

Enwright, Nicholas M.

Eo, YangDam

Eppelbaum, Lev

Erdélyi, Ján

Eric K., Davis

Erinjery, Joseph

Erives, Hector

Ermakov, Dmitry

Ermakov, Stanislav Alexandrovich

Ermoshkin, Aleksey

Ermoshkin, Alexey V.

Ernstson, Kord

Eroglu, Orhan

Er-Raki, Salah

Erudel, Thierry

Eshagh, Mehdi

Eskandari Torbaghan, Mehran

Esmaili, Rebekah

España, Raquel Martínez

Espejel, Ileana

Espindola, Giovana

Espinosa, Víctor

Espinoza, Carlos Zúñiga

Espinoza, Gonzalo

Esposito, Giuseppe

Esposito, Salvatore

Esselborn, Saskia

Essenfelder, Arthur Hrast

Essien, Etido

Estel, Stephan

Estévez, Javier Gualda

Eugenio, Francisco

Euillades, Pablo

Eulenfeld, Tom

Eustáquio Dantas Chaves, Michel

Eva, Mihail 
Evans, Robin

Evaristo, Raquel

Evelpidou, Niki

Even, Markus

Everett, Mark

Evsukoff, Alexandre G.

Exertier, Pierre

Eyji Sano, Edson

Ezcurra, Exequiel

Ezzahar, Jamal

Ezzati, Sattar

F. De Arruda, Henrique

Fabris Rotelli, Inger

Fabris, Massimo

Faccini, Francesco

Faggella, Marco

Fagin, Todd

Fagua, J. Camilo

Fairen, Alberto

Fairley, Iain

Faivre, Robin

Fajardo, Mario

Falarz, Malgorzata

Falcão, Ana Paula

Falco, Nicola

Falcone, James A.

Falini, Antonella

Fallati, Luca

Fallavollita, Paolo

Fallows, Richard

Famoso, Fabio

Fan, Deqin

Fan, Hongchao

Fan, Jinlong

Fan, Lei

Fan, Wenyi

Fan, Xingwang

Fanfarillo, E.

Fang, Guangyang

Fang, Hongliang

Fang, Jianwu

Fang, Rongxin

Fang, Shibo

Fang, Tzu-Wei

Fang, Xuan

Fang, Yuanhao

Fang, Zhigeng

Farahani, Behzad V.

Farahnakian, Fahimeh

Faraslis, Ioannis

Fardoulis, John
Fareed, Nadeem

Fargnoli, Mario

Farguell Caus, Angel

Farguell, Angel

Farias, Wendell Rondinelli Gomes

Faridani, Farid

Faridatul, Mst Ilme

Farkas, Jenő Zsolt

Farlik, Jan

Farolfi, Gregorio

Farthing, Matthew

Faruolo, Mariapia

Farzaneh, Saeid

Fascista, Alessio

Fassi, Francesco

Fassnacht, Steven

Fatma, Jebri

Fatolazadeh, Farzam

Fauchard, Cyrille

Fava, Maria Clara

Favali, Paolo

Fawky, Abdelfattah

Fayad, Abbas

Fayad, Ibrahim

Fayne, Jessica

Fedeli, Alessandro

Federico, Stefano

Fedorov, Alexander

Fedotov, Alexander

Fei, Tai

Feito Higueruela, Francisco R.

Feitosa, Raul

Feizizadeh, Bakhtiar

Feldens, Peter

Felicetti, Leonard

Felipe, Guzman

Feltynowski, Marcin

Fencik, Robert

Feng, Dongmei

Feng, Guangcai

Feng, Guang-Liang

Feng, Li

Feng, Min

Feng, Quanlong

Feng, Ruitao

Feng, Sheng

Feng, Tian

Feng, Xuezhi

Feng, Yuhao

Feofilov, Artem

Feoktistova, Ekaterina 
Feoli, Enrico

Fera, Martin

Ferecatu, Marin

Feret, Jean Baptiste

Fernandes, Carlos Marcello Dias

Fernandes, Francisco

Fernandes, Joana

Fernandes, Maria Rosário

Fernández De Córdoba, Rocío Mora

Fernández Jaramillo, Arturo Alfonso

Fernandez, Helena

Fernandez, Laura

Fernandez, Margarita

Fernández, María García

Fernández, Susana

Fernández, Tomás

Fernández., Ignacio

Fernández-Campoy, Juan Miguel

Fernández-García, Victor

Fernández-Hernández, Jesús

Fernández-Lozano, Javier

Ferracini, Chiara

Ferrandez-Villena, Manuel

Ferrandiz, Jose Manuel

Ferrando, Ilaria

Ferrant, Sylvain

Ferrarini, Andrea

Ferrario, Maria Francesca

Ferraris, Stefano

Ferreira, Ana Navarro

Ferreira, André G.

Ferreira, Artur

Ferreira, Hugo

Ferreira, Karine Reis

Ferreira, Manuel

Ferreira, Vagner G.

Ferrentino, Emanuele

Ferrero, Alejandro

Ferretti, Alessandro

Ferrier, Graham

Fhager, Lars Ohlsson

Fiala, Pavel

Fialho, Vitor

Fialko, Yuri

Fiandrotti, Attilio

Fiani, Margherita

Fickert, Thomas

Fidani, Cristiano

Fidas, Christos

Field, Richard

Fierek, Szymon
Figueiredo Prado, Luciana

Figueiredo, Cosme Alexandre O. B.

Fikfak, Alenka

Fila, Gianni

Filchev, Lachezar

Filchev, Lachezar Hristov

Filimonov, Mikhail Yur'evich

Filippini, Tommaso

Filipponi, Federico

Filippov, N. N.

Filippucci, Paolo

Filizola, Naziano

Filjar, Renato

Finke, Ullrich

Finkenbiner, Catherine E.

Finlon, Joseph

Fiorentin, Pietro

Fiori, Robyn

Fiorino, Steven

Fiorito, Francesco

Fiorucci, Paolo

Fiscante, Nicomino

Fisch, Gilberto

Fischer, Andrea

Fischer, Andrew

Fisher, Daniel K.

Fitch, G. Michael

Fizesan, Raul

Flamini, Enrico

Fletcher, Steven

Flocas, Helena

Flor-Blacnco, Germàn

Flores Romero, Juan Jose

Flores Tapia, Daniel

Flores, Gerardo

Floris, Antonio

Floris, Mario

Fofonova, Vera

Foivos, Diakogiannis

Fok, Hok Sum

Folini, Doris Sylvia

Folley, Christopher N.

Foltz, Gregory

Fonseca, Ana

Fonseca-Luengo, David

Fontan Bouzas, Angela

Fontan, Fernando Perez

Fontana, Simone

Fontanelli, Giacomo

Fonte, Joao

Fontul, Simona 
Foolad, Foad

Ford, Anabel

Ford, David A.

Ford, John

Ford, Phillip

Forero, Manuel

Forghani, Mojtaba

Forlani, Gianfranco

Forlin, Paolo

Formisano, Antonio

Fornacca, Davide

Foroughnia, Fatemeh

Forristall, George Z.

Förster, Kristian

Forte, Giovanni

Foti, Giandomenico

Foucher, Philippe

Foucher, Pierre-Yves

Foucher, Samuel

Fountoulakis, Ilias

Fournier, Georges

Fox, Neil I.

Fragkos, Konstantinos

Fraisse, Renaud

Francesca Carfora, Maria

Francesca, Calastrini

Francesco, Basile

Franch, Belen

Francioni, Mirko

Francone, Antonio

Franquesa, Magí

Franz, Bryan A.

Franzen, Dave

Franzini, Marica

Frappart, Frédéric

Fraser, Benjamin T.

Fraštia, Marek

Frech, Michael

Fredericksen, Todd

Free, Gary

Freeborn, Patrick

Freeman, Vahid

Frei, Michaela

Freitas, Pedro Garcia

Frejlichowski, Dariusz

Frelich, Lee

French, Andrew N.

French, Nancy

Freni, Gabriele

Freret-Lorgeril, Valentin
Frery, Alejandro

Fretwell, Peter

Frey, Julian

Frey, Othmar

Frey, Richard

Frey, Sandor

Frías, Ernesto

Friberg, Mariel D.

Friedel, Matthias

Friedrich, Martina M.

Friedt, Jean-Michel

Friesen, John

Fritsch, Dieter

Frodella, William

Frolov, Denis

From, Andrew

Fron, Adam

Frontoni, Emanuele

Frost, Gerald

Fryskowska, Anna

$\mathrm{Fu}$, Dongjie

$\mathrm{Fu}$, Haiqiang

$\mathrm{Fu}$, Peng

$\mathrm{Fu}$, Ying

$\mathrm{Fu}$, Yuanyuan

$\mathrm{Fu}$, Yuning

$\mathrm{Fu}$, Yuyun

Fuentes Reyes, Mario

Fuentes, Valeriano Méndez

Fugazza, Cristiano

Fujibe, Fumiaki

Fujimura, Atsushi

Fujisaki, Kiyotaka

Fujita, Ichiro

Fujita, Kohei

Fujita, Mikiko

Fukunaga, Atsuko

Funari, Marco Francesco

Funatsu, Beatriz M.

Furlani, Stefano

Fursov, Vladimir

Furuya, Masato

Fustos, Ivo

Fyllas, Nikolaos

Gabara, Grzegorz

Gábor, Marián

Gabriel, Jose Luis

Gadal, Sebastien

Gade, Martin

Gadedjisso-Tossou, Agossou

Gądek, Wiesław 
Gafurov, Artur M.

Gaggero, Tomaso

Gagliardi, Francesca

Gagliardi, Gianfranco

Gagliardi, Valerio

Gai, Shaoyan

Gaikovich, Konstantin P.

Gailler, Lydie

Gaj, Renata

Gajski, Dubravko

Gaju, Oorbessy

Gala, Tekleab S.

Gałaj, Jerzy

Galante, Francesco

Galdies, Charles

Galeone, Ciro

Galgamuwe A., Pabodha G.

Gallagher, Neal

Gallant, John

Gallardo, Marta

Gallay, Michal

Gallego-Elvira, Belen

Gallegos-Funes, Francisco

Galo, Maria De Lourdes B. Trindade

Galvanin, Edinéia Aparecida Dos Santos

Galvao, Lenio

Galve, Joan Miquel

Galve, Jorge Pedro

Gama, Fábio Furlan

Gambella, Filippo

Gamcova, Maria

Gan, Guojing

Ganagina, Irina

Ganas, Athanassios

Ganatsas, Petros

Ganci, Gaetana

Ganesh C., Bora

Ganetsos, Theodore

Ganguli, Swetava

Ganyushkin, Dmitry A.

Gao, Bocai

Gao, Chunchun

Gao, Fan

Gao, Feng

Gao, Haiyang

Gao, Han

Gao, Hongmin

Gao, Jiangbo

Gao, Jie

Gao, Jinlong

Gao, Junfeng
Gao, Jungang

Gao, Liang

Gao, Lianru

Gao, Lin

Gao, Meng

Gao, Peichao

Gao, Qi

Gao, Shanhong

Gao, Shuai

Gao, Xin

Gao, Yan

Gao, Zhi

Gao, Zhiqiu

Gao, Zhongming

Gara, Tawanda

Garaba, Shungudzemwoyo

Garane, Katerina

Garbero, Valeria

García Floriano, Andrés

Garcia Sanchez, Jesus

Garcia, Angel Pontin

García, David Hidalgo

Garcia, Matthew

García, Omaira

Garcia, Pilar

García-Arnay, Ángel

García-Asenjo, Luis

García-Giménez, Rosario

Garcia-Gutierrez, Jorge

García-Haro, Francisco Javier

García-Lamont, Farid

García-Mateos, Ginés

Garcia-Melendez, Eduardo

Garcia-Molsosa, Arnau

García-Morales, Ricardo

Garcia-Pineda, Oscar

García-Ruíz, Francisco

Garcia-Santos, Vicente

Garcia-Selles, David

Garde, Eva

Gardiner-Garden, Robert

Gardner, John

Garea, Alberto S.

Garg, Sourav

Gargano, Marco

Garioud, Anatol

Garnero, Gabriele

Garrett, Joseph L

Garrett, Kevin J.

Garrido, I.

Garrido-Villén, Natalia 
Garstang, Michael

Garvanov, Ivan

Garvanova, Magdalena

Garza, Paolo

Garza-Castañón, Luis E.

Garza-Gil, María Dolores

Garza-Pérez, Joaquin Rodrigo

Garzelli, Andrea

Gašinec, Juraj

Gašparac, Goran

Gasparatos, Dionisios

Gašparović, Mateo

Gastauer, Markus

Gatto, Elisa

Gavahi, Keyhan

Gavrilov, Boris

Gavrilov, Milivoj B.

Gay, Alan

Gay, Michel

$\mathrm{Ge}$, Maorong

Ge, Yingchun

Ge, Yunfeng

Ge, Zhenming

Gebremichael, Esayas

Gedefaw, Abebaw Andarge

Gediga, Krzysztof

Gee, Jeffrey

Geiger, Alain

Geli, Hatim

Gella, Getachew Workineh

Gelli, Samuele

Gemitzi, Alexandra

Genareau, Kimberly

Gencaga, Deniz

Genchi, Sibila A.

Genesio, Lorenzo

Geng, Jie

Gennarelli, Gianluca

Gennaro, Massimo

Genova, Antonio

Gensemer, Stephen

Gentilucci, Matteo

Genzano, Nicola

Georganos, Stefanos

George, Sony

Georges, Stienne

Georgescu, Puiu Lucian

Georgiadis, Charalampos

Georgiadis, Teodoro

Georgiev, Christo

Georgiou, Nikos
Georgopoulos, Andreas

Gerald, Blasch

Gerasimov, Vladislav V.

Gerbeth, Daniel

Gerhard, Gerold

Gerlach, Christian

Germani, Chiara

Ghaderpour, Ebrahim

Ghaffarian, Saman

Ghafouri-Azar, Mona

Ghajar, Shayan

Ghanmi, Helmi

Gharechelou, Saeid

Gharehchahi, Saeideh

Gharineiat, Zahra

Ghavidel, Ali

Gherm, Vadim E.

Ghiasi, Yusof

Ghilain, Nicolas

Ghods, Abdolreza

Gholamreza, Anbarjafari

Gholizadeh, Asa

Ghorbanzadeh, Omid

Ghosal, Sambuddha

Ghosh, Tilottama

Giacomini, Anna

Gialampoukidis, Ilias

Giani, Paolo

Giannico, Vincenzo

Giannini, Fernanda

Giannone, Francesca

Giannopoulos, Michalis

Giano, Salvatore Ivo

Giardino, Claudia

Gibson, John

Gibson, Lesley

Gichenje, Helene

Gienko, Gennady

Gierens, Klaus

Gignac, Charles

Gigovic, Ljubomir

Gil, Arturo Aparicio

Gilbert, Fabien

Gilewski, Paweł

Gilewski, Paweł Grzegorz

Gilja, Gordon

Gill, Nathan

Gillan, Jeffrey K.

Gillerson, Alexander

Gillotay, Didier

Gil-Świderska, Agnieszka 
Gimona, Alessandro

Gioia, Ciro

Gioia, Dario

Giordan, Daniele

Giovanacci, David

Giovanni, Adriano Di

Giovanni, Argenti

Giovanni, Fabbrocino

Gips, Bart

Girelli, Valentina Alena

Girina, Olga

Girolamo-Neto, Cesare Di

Gislum, René

Gitas, Ioannis

Gitis, Valeri

Gittings, John

Giudicepietro, Flora

Giuffrida, Gianluca

Giuliani, Chiara

Giuliani, Roberta

Gjevestad, Jon Glenn

Gkyrtis, Konstantinos

Glaser, John

Gleich, Dusan

Glein, Robért

Gligor, Adrian

Glińska-Lewczuk, Katarzyna

Głowacki, Tadeusz

Głowacz, Adam

Glowienka, Ewa

Głowiński, Sebastian

Gnyawali, Kaushal Raj

Go, Sujung

Gobeawan, Like

Gobo, João Paulo Assis

Gobron, Nadine

Goddijn-Murphy, Lonneke

Godec, Ranka

Godio, Alberto

Godone, Danilo

Goebbels, Steffen

Goeking, Sara

Goela, Priscila Costa

Goerl, Roberto Fabris

Gogineni, Vinay Chakravarthi

Gohar, Ghazaryan

Gohin, Francis

Gojda, Martin

Gokaraju, Balakrishna

Gokon, Hideomi

Golombek, Matthew P.
Golston, Levi

Goltz, Douglas M.

Golyandina, Nina

Gomes, Alberto

Gomes, Alessandra

Gomes, Eduardo

Gomes, Natanael Rodrigues

Gómez Déniz, Luis

Gomez, Christopher

Gomez, Cristina

Gómez, Luis

Gomez-Dans, Jose

Gomez-Donoso, Francisco

Gomez-Espinosa, Alfonso

Gómez-Gutiérrez, Alvaro

Gómez-Ortiz, David

Gomez-Pazo, Alejandro

Gomiz Pascual, Juan Jesús

Gonçalves Antunes, João Francisco

Gonçalves, Ana Cristina

Gonçalves, Gil

Gonçalves, João Francisco

Gonçalves, José Alberto

Gonçalves, Luísa

Goncalves, Paulo

Gonçalves, Rodrigo M.

Gong, Adu

Gong, Hainan

Gong, Jie

Gong, Shaoqi

Gong, Wenyu

Gong, Yun

González De Audicana, María

González, Carlos

Gonzalez, Luciano

Gonzalez, Marcela

Gonzalez, Rodrigo

Gonzalez, Sergi

González-Hidalgo, Manuel

González-Jaramillo, Víctor Hugo

González-Núñez, Rubén

González-Piqueras, José

González-Sosa, Enrique

González-Villanueva, Rita

Gonzalo Orden, Hernán

Gonzalvez, Pablo Rodriguez

Good, Stephen

Goodin, Christopher T.

Goodin, Douglas G.

Goodrich, Gregory B.

Gopalakrishnan, Gnanachandrasamy 
Gopinath, Girish

Gorbenkova, Elena

Gorbunov, Michael

Gordillo-Vazquez, Francisco J.

Górecki, Tomasz

Gorgan, Dorian

Goring, Derek

Göritz, Anna

Gorroño, Xavier

Gotor, Alicia Ayerdi

Gotovac, Sven

Gottschall, Julia

Goulet, Denis

Goulette, Francois

Gourbesville, Philippe

Gourgiotis, Anestis

Gournia, Charikleia

Govedar, Zoran

Govedarica, Miro

Govil, Himanshu

Goyanes, Gabriel

Gozdowski, Dariusz

Grabowska, Magdalena

Gracia Prieto, Francisco Javier

Grafton, Miles

Gragnani, Gian Luigi

Graham, Garrett

Grammalidis, Nikos

Grams, Heather

Granados-Muñoz, María José

Granata, Donatella

Grasso, Nives

Gratien, Jean-Marc

Graves, Tabitha

Graziani, Alberto

Graziano, Maria Daniela

Greco, Filippo

Grecu, Mircea

Green, Christopher M.

Green, David R.

Gregori, Giovanni

Grenzdörffer, Görres

Greybush, Steven

Grgić, Marijan

Gribanov, K. G.

Grieco, Giuseppe

Grießbach, Sabine

Griffin, Amy

Griffin, Debora

Griffith, Henry

Griffiths, David
Griggs, Erin

Grigoras, Ion

Grigoriadis, Vassilios N.

Grigoriev, Gennady I.

Grigorieva, Elena A.

Grigorii P., Kokhanenko

Grilli, Eleonora

Gritsevich, Maria

Grochla, Krzysztof

Gröger, Gerhard

Groh, Andreas

Gromek, Artur

Gross, Barry

Groß, Silke

Große-Stoltenberg, André

Grossmann, Katja

Grudzielanek, Anja Martina

Grulke, Nancy E.

Grunert, Brice

Grunwald, Grzegorz

Gruszczynski, Wojciech

Grybas, Heather

Gryś, Sławomir

Grytsai, Asen

Grzempowski, Piotr

Gu, Jianyu

$\mathrm{Gu}$, Lingjia

$\mathrm{Gu}$, Shengfeng

$\mathrm{Gu}$, Xiaohe

$\mathrm{Gu}$, Yalong

Gu, Zhu-Jun

Guallpa, Mario

Guan, Lei

Guan, Qingyu

Guariglia, Emanuel

Guarnieri, Andrea Monti

Gubbi Sadashiva, Thejas

Guccione, Pietro

Gucma, Maciej

Gucunski, Nenad

Guerova, Guergana

Guerra Cobian, Victor Hugo

Guerra, José De Los Santos

Guerra, Juan

Guerrero, Jesús

Guerrero-Ginel, José Emilio

Guerrero-Rascado, Juan Luis

Guerriero, Luigi

Guevara, Mario

Guglielmin, Mauro

Guida, Domenico 
Guijo-Rubio, David

Guilbert, David

Guilhot, Denis

Guillaume, Giroir

Guillaume, Mireille

Guillén, María Luz

Guilloteau, Clément

Guinard, Stephane

Guinau Sellés, Marta

Guindon, Luc

Gulbeyaz, Onder

Guliaev, Roman

Gultepe, Ismail

Gumaei, Abdu

Gumbmann, Frank

Gumma, Murali Krishna

Gunawan, Teddy Surya

Gunn, Grant

Gunter, William Scott

Guo, Baofeng

Guo, Bin

Guo, Dejun

Guo, Hong

Guo, Jianping

Guo, Jifa

Guo, Jinyun

Guo, Longxiang

Guo, Qingchun

Guo, Qinghua

Guo, Wen-Dar

Guo, Wenfei

Guo, Xiaojun

Guo, Xulin

Guo, Yuanyue

Guo, Yulong

Guoan, Yin

Gupta, Lalit

Gurbuz, Ali C.

Gurdak, Radoslaw

Gurlin, Daniela

Gusarov, Artyom

Gusti, Mykola

Gutierrez, Antonio

Gutiérrez, Eduardo Quevedo

Gutierrez, Miguél Ángel

Gutjahr, Karlheinz

Guttikunda, Sarath K.

Guzy, Artur

Guzzi, Donatella

Gwenzi, David

Gyawali, Buddhi
Gyawali, Madhu

Ha, Lan Thanh

Ha, Nguyen Thi Thu

Ha, Wonsook

Haack, Barry

Haagsma, Marja

Habaragamuwa, Harshana

Habib, Adnane

Habyarimana, Ephrem

Hachaj, Tomasz

Hachoł, Justyna

Hacker, Joshua

Hackney, Christopher R.

Haddad, Bouchra

Hadjisolomou, Ekaterini

Hadush, Meresa

Hagag, Ahmed

Hagan, Daniel Fiifi Tawia

Hagen, Martin

Haghshenas, Sina Shaffiee

Haghverdi, Amir

Haginoya, Shigenori

Hajduk, Marcin

Hajdukiewicz, Hanna

Hajj, Marcel El

Hajj, Mohammad Ei

Halabuk, Andrej

Haladová, Zuzana Berger

Halbac-Cotoara-Zamfir, Rares

Haldorsen, Jakob

Halenka, Tomáš

Halicioglu, Kerem

Halin, Alfian Abdul

Hall, Joanne

Halla, Jamie

Hallik, Lea

Hallo, Miroslav

Halmai, Ákos

Ham, Namhyuk

Hamada, Yuki

Hamal, Kalpana

Hamed, Ali

Hamedi, Amirmasoud

Hameed, Khurram

Hameed, Maysoun

Hamilton, Murray

Hamm, Nicholas

Hammad, Mohamed Adel

Hammer, Marcus

Hämmerling, Mateusz

Hammond, Matthew 
Hamran, Svein-Erik

Han, Bing

Han, Chin-Chuan

Han, Congzheng

Han, Cunbo

Han, Dawei

Han, Dejun

Han, Ge

Han, Heechan

Han, Hyangsun

Han, Il Song

Han, Kevin

Han, Peng

Han, Songjun

Han, Soo Hee

Han, Xianhua

Han, Xiao

Han, Youkyung

Han, Yuxing

Han, Zheng

Han, Zhi

Han, Zhigang

Hanaeus, Jörgen

Hand, Emily

Haneberg, William C.

Hanink, Dean M.

Hansen, Morten. W.

Hanson, Brian

Hantson, Wouter

Hanus, Paweł

Hao, Dalei

Hao, Pengyu

Hao, Xianjun

Hao, Yongqiang

Hao, Zengzhou

Hapke, Bruce

Haq, Mohd

Harabaglia, Paolo

Haraguchi, Akira

Harber, David

Hardenbicker, Ulrike

Harding, Chris

Harding, Richard

Hardy, Andy

Hardy, Ryan

Hare, Trent M.

Hariharan, Jeanette

Harikumar, Aravind

Harirchian, Ehsan

Hariri-Ardebili, Mohammad Amin

Harkat, Houda
Harkey, Monica

Harmel, Tristan

Harris, Lucas

Harris, Trevor

Haruki, Oshio

Hasager, Charlotte Bay

Hasan, Emad

Hasan, Sarah

Hasanlou, Mahdi

Hasanzadeh, Sogand

Hashemi-Beni, Leila

Hasheminasab, Seyyed Meghdad

Hashiguchi, Hiroyuki

Hassaballah, M.

Hassan, Ali

Hassan, Quazi K.

Hassan, Salwa

Hata, Fernando Teruhiko

Hatakeyama, Shiro

Hauchecorne, Alain

Haule, Kamila

Hauschild, Andre

Hauser, Danièle

Hautecoeur, Olivier

Havenith, Hans-Balder

Havivi, Shiran

Hawbaker, Todd

Hawes, Ian

Hawryło, Paweł

Hayasaka, Hiroshi

Hayashi, Syugo

Hazbavi, Zeinab

Hazelton, Pamela

Hazelwood, Richard

Hazra, Debapriya

$\mathrm{He}$, Baojie

$\mathrm{He}$, Cenlin

He, Changyong

He, Fangning

He, Jianhui

He, Kaifei

He, Mingzhu

$\mathrm{He}$, Shengping

He, Shuangyan

$\mathrm{He}$, Siming

$\mathrm{He}$, Tao

He, Wei

He, Xiaoxing

He, Xiufeng

$\mathrm{He}$, Yaqian

$\mathrm{He}$, Yi'nan 
He, Yijun

He, Yuan

He, Yunze

Head, James

Heald, Gary J.

Healey, Sean

Hearn, Thomas

Heath, Linda S.

Hecker, Christoph A.

Hedayati, Faraz

Heddam, Salim

Hedley, John D.

Hefferan, Kevin Patrick

Hegler, Sebastian

Heidarpour Shahrezaei, Iman

Heiden, Uta

Heilman, Philip

Heinze, Thomas

Heiselberg, Henning

Hejmanowski, Ryszard

Hejna, Monika

Held, Philipp

Helm, Veit

Helmi, Ghanmi

Helzel, Thomas

Hemanth, Jude

Henchiri, Malak

Henebry, Geoffrey

Hénin, Riccardo

Henriques, Renato Filipe

Henry, Mary

Henryk, Lasota

Hens, Luc

Hepplewhite, Chris

Heras, Jónathan

Hereher, Mohamed

Hering-Bertram, Martin

Herman, Grigore

Herman, Grigore Vasile

Herman, Lukáš

Hermans, Thomas

Hernández Sosa, José Daniel

Hernandez, Oliver Gutierrez

Hernández-Hernández, José Luis

Hernandez-Leal, Pedro A.

Hernández-López, David

Hernández-Stefanoni, José Luis

Herndon, Derrick

Heron, Malcolm

Herrera, Dimitris

Herrera, Jesus Antonio Sosa
Herrera, Pedro Javier

Herrero, Hannah

Herrero-Jiménez, Carlos Miguel

Herrmann, Ittai

Herrmann, Michele

Herrmann, Stefanie

Herschel, Reinhold

Herschfelt, Andrew

Hertig, Elke

Hervás-Gámez, Carmen

Hesham El, Askary

Heshmati-alamdari, Shahab

Hesselbarth, Anja

Hessing-Lewis, Margot L.

Hethcoat, Matthew G.

Hettiarachchi, Eshani

Hewitt, Bailey

Hewson, Michael

Hewson, Robert

Heyduk, Adam

Heymsfield, Andrew J.

Hibbitts, Karl

Hidy, George

Hieronymi, Martin

Higginbottom, Thomas

Higgins, Suzanne

Higuchi, Atsushi

Hilburn, Kyle A.

Hill, Michael

Hill, Peter

Hill, Victoria J.

Hillger, Don

Hilt, Attila

Himi, Mahjoub

Hinostroza Sáenz, Israel David

Hinterleitner, Alois

Hiraishi, Tetsuya

Hirata, Yasumasa

Hird, Jennifer

Hirooka, Yoshihiro

Hiroshi, Matsuyama

Hirschmugl, Manuela

Hisaki, Yukiharu

Hislop, Samuel

Hladnik, David

Hlaváčová, Ivana

Hmmimina, Gabriel

Hnatiuc, Mihaela

Hnilicka, Frantisek

Ho Tong Minh, Dinh

Ho, Ching-Hsien 
Ho, Chung-Ru

Ho, Hann Woei

Ho, Tan-Jan

Hoang, Nguyen

Hoang, Nguyen Tien

Hoang, Quoc-Dong

Hoang, Thanh Van

Hochberg, Eric

Hocke, Klemens

Hodges, Richard

Hoeksema, Bert

Hoffmann, Joern

Hoffmann, Lars

Hofierka, Jaroslav

Hofmann, Peter

Höger, Marek

Hohensinn, Roland

Hole, Lars Robert

Holman, Fenner H.

Holobaca, Iulian Horia

Holst, Christoph

Holzapfel, Eduardo A.

Holzwarth, Stefanie

Homma, Koki

Honda, Takumi

Honeine, Paul

Hong, Bo

Hong, Chang-Ki

Hong, Danfeng

Hong, Haoyuan

Hong, Ilyoung

Hong, Sang-Hoon

Honniball, Casey

Honório, Leonardo De Mello

Hontoria, Leocadio

Hooke, Janet

Hopmans, Jan

Hoppe, Edward

Horel, Ágota

Horemuz, Milan

Horn, Berthold

Hornero, Alberto

Horng, Shi-Jinn

Horstrand, Pablo

Hoshikawa, Keisuke

Hoshino, Buho

Hossain, Azad

Hossain, Md Alamgir

Hossain, Md Zakir

Hossain, Mohammad Shawkat

Hosseini, Saeid
Hoth, Julian

Hou, Dongyang

Hou, Hao

Hou, Jinliang

Hou, Tsung-Chin

Hou, Weizhen

Hou, Yu

Houghton, Isabel

Houlié, Nicolas

Houška, Jakub

Houssein, Essam H.

Houweling, S.

Howard, Susan L.

Høyer Svendsen, Synne

Hrdalo, Ines

Hsieh, Ping-Cheng

Hsieh, Yu-chung

Hsin, Kun-Yi

Hssaisoune, Mohammed

$\mathrm{Hsu}$, Pochun

$\mathrm{Hu}$, Andong

$\mathrm{Hu}$, Baoxin

$\mathrm{Hu}$, Bifeng

$\mathrm{Hu}$, Caihong

$\mathrm{Hu}, \mathrm{Di}$

$\mathrm{Hu}$, Gaoge

$\mathrm{Hu}, \mathrm{Han}$

$\mathrm{Hu}$, Jiaxi

$\mathrm{Hu}$, Jingliang

$\mathrm{Hu}$, Jun

Hu, Jyr-Ching

$\mathrm{Hu}$, Luojia

$\mathrm{Hu}$, Qiong

$\mathrm{Hu}$, Tangao

$\mathrm{Hu}$, Zhan-Yi

$\mathrm{Hu}$, Zhenghua

$\mathrm{Hu}$, Zhigang

$\mathrm{Hu}$, Zhongwen

$\mathrm{Hu}$, Zhongyang

Hua, Xiaoqiang

Huang, Bo

Huang, Chang

Huang, Changchun

Huang, Changping

Huang, Ching-Jer

Huang, Ching-Yuang

Huang, Chiou-Jye

Huang, Chunbo

Huang, Conghong

Huang, Daji

Huang, Daquan 
Huang, Faming

Huang, Feixiong

Huang, Gordon

Huang, Guo-Hao

Huang, Guohe

Huang, Haiyan

Huang, Haocai

Huang, Hong

Huang, Hongwei

Huang, Huabing

Huang, Huaguo

Huang, Jianxi

Huang, Jor-Man

Huang, Kai

Huang, Ke

Huang, Lanqing

Huang, Lingcao

Huang, Qihuan

Huang, Qiongyu

Huang, Rong

Huang, Shaoguang

Huang, Shaoli

Huang, Shih-Ming

Huang, Ting-Hsuan

Huang, Weimin

Huang, Wenli

Huang, Xiao

Huang, Xiaodong

Huang, Yan

Huang, Yanbo

Huang, Yaohuan

Huang, Yishuo

Huang, Yiyi

Huang, Yuhan

Huber, Stefan Huber

Huesca, Margarita

Huffman, George

Hughes, Nick

Huh, Morang

Huh, Yong

Hui, Fengming

Hulsey, Leroy

Humphreys, John

Hung, Ming-Chih

Hunt, Eric

Hunt, Samuel

Hunter, David

Hunter, Molly E.

Huo, Chunlei

Huo, Lian-Zhi

Huo, Xingliang
Huq, Md. Enamul

Hur, Jina

Hurtík, Petr

Husak, Greg

Hussain, Ekbhal

Hussain, Muhammad

Hussain, Niamat

Hussein, Hussam

Hutchison, Keith

Hutley, Lindsay

Hutorowicz, Andrzej

Huynh, Thanh-Canh

Huynh-The, Thien

Huzui-Stoiculescu, Alina

Hvidegaard, Sine Munk

Hwang, Dong-Hwan

Hwang, Jisoo

Hwang, Paul

Hwang, Yoola

$\mathrm{Hwu}$, Jih Ru

Hyer, Edward

Hysa, Artan

Hyun, Chang-Uk

Iacobucci, Giulia

Iacovazzi, Robert

Ialongo, Iolanda

Iannella, Mattia

Ianos, Ioan

Ibrahim, Ahmed Mokhtar Nagy

Ibrahim, Sa' ad

Ichii, Kazuhito

Ichikawa, Kaoru

Iddianozie, Chidubem

Idzanovic, Martina

Ienco, Dino

Ientilucci, Emmett

Igne, Benoît

Iguchi, Masato

Ihamouten, Amine

Iio, Atsuhiro

Iizuka, Satoshi

Ikawa, Hiroki

Ilangakoon, Nayani

Iliev, Teodor B

Ilieva, Zhenya

Ilori, Christopher

Ilyin, Alexey

Im, Sergey

Imai, Nilton

Imam, Rayan

Imasu, Ryoichi 
Imbrenda, Vito

Imperatore, Pasquale

Inagaki, Atsushi

Inchingolo, Giuseppe

Indamar, Anand

Inggs, Michael

Inghilesi, Roberto

Inglot, Adam

Inia, Soto

Inkinen, Tommi

Innocenti, Carlo

Intzes, Ioannis

Inzerillo, Laura

Ioana, Cornel

Ioannidis, Konstantinos

Ioannou, Stelios

Iodice, Antonio

Ioki, Keiko

Ioras, Florin

Iordache, Marian-Daniel

Iorga, Gabriela

Iosub, Marina

Ippoliti, Carla

Iqbal, Irfan Akhtar

Iqbal, Umar

Iriarte, Juan

Irie, Mitsuteru

Irimescu, Anisoara

Irisarri, J. Gonzalo

Irisov, Vladimir

Irteza, Syed M.

Irwin, Louis

Iryna, Piestova

Isar, Alexandru

Ishida, Haruma

Ishidaira, Hiroshi

Ishikawa, Tadashi

Islam, Kamrul

Islam, Kazi Aminul

Islam, Mohammad Nurul

Islam, Muhammad Aminul

Ismail, Adiel

Ismail, Mohamed

Issa, Hamza

Issa, Salem

Ita, Eyo

Itahashi, Syuichi

Itakura, Kenta

Ito, Seiya

Iungo, Giacomo Valerio

Ivajnšič, Danijel
Ivan, Pires

Ivanov, Atanas

Ivanov, Danil

Ivanov, S. I.

Ivanova, Ekaterina

Ivanova, Ivana

Ivanović, Sanjin

Ivanovic, Stefan S.

Ivashov, Sergey I.

Ivetic, Vladan

Ivezic, Vedran

Ivits, Eva

Ivonin, Dmitry

Ivšinović, Josip

Iwana, Brian Kenji

Iwasaki, Kenta

Iwasaki, Suginori

Izaguirre, Alberto

Izhitskiy, Alexander

Izquierdo Verdiguier, Emma

Izquierdo, Estibaliz Martinez

Izydorczyk, Jacek

J. Daniels, David

J. H. Dodd, Thomas

J. Vega, Daniel

Jabari, Shabnam

Jacko, Stanislav

Jackson, Joshua J.

Jackson, Rhett

Jacob, Maria

Jacobsen, Karsten

Jacygrad, Ewelina

Jadidoleslam, Navid

Jaferzadeh, Keyvan

Jagat Dwipendra, Ray

Jagoda, Marcin

Jahnke, Mathias

Jain, Ankita Deepak

Jaiswal, Neeru

Jakob, Matthias

Jakovljevic, Gordana

Jaligot, Rémi

Jalil, Bushra

Jaliu, Codruta

Jamali, Sadegh

Jamei, Yashar

James, Todd

Jameson, Arthur

Jamet, Cédric

Jamshidi, Maziar

Jamshidi, Sajad 


Jan, Chyan-Long
Janák, Juraj
Janc, Dejan
Janet, Nichol
Jang, Gil-Jin
Janicka, Joanna
Janicki, Grzegorz
Jánosi, Imre M.
Janowski, Artur
Janowski, Lukasz
Jansen, Robert W.
Jansing, David
Janssen, Thomas A. J.
Jantz, Patrick A.
Janus, Jarosław
Jarchow, Christopher J.
Jarman, David
Jarocińska, Anna
Jasiewicz, JarosŁaw
Jasinski, Radoslaw
Jaskuła, Joanna
Jaskulski, Marcin
Javed, Zeeshan
Javeed, Nadeem
Javeed, Tehseen
Javier Cardenal, Francisco
Javornik, Tomaz
Jaworowski, Cheryl
Jaworska, Tatiana
Jayathunga, Sadeepa
Jebri, Fatma
Ji, Ledlicka, Karel
Ji, Zhengeng
Jeganathan, Chockalingam
Jelének, Jan
Jen, Chia-Hung
Jenerowicz, Agnieszka
Jeng, Yih
Jennifer A., Schulien
Jensen, Daniel
Jensen, Katherine
Jeong, Myeong-Hun
Jeong, Young-Seob
Jer Jaw, Jen

Jia, Hongguo

Jia, Kun

Jia, Shenyue

Jia, Yong

Jia, Yuanyuan

Jian, Muwei

Jiang, Bin

Jiang, Changhui

Jiang, Chongya

Jiang, Junjun

Jiang, Junle

Jiang, Lingmei

Jiang, Ruinian

Jiang, San

Jiang, Wei

Jiang, Weiguo

Jiang, Xiaojun

Jiang, Yan

Jiang, Zhongshan

Jianjiang, Wang

Jiao, Licheng

Jiao, Quanjun

Jiao, Ziti

Jiateng, Guo

Jie, Dou

Jiménez Ballesta, Raimundo

Jiménez López, Jesús

Jimenez, Cristofer

Jimenez, José

Jiménez, Marcos

Jiménez, Miguel Clavijo

Jimenez, Pedro A.

Jiménez-Muñoz, Juan Carlos

Jimeno-Saez, Patricia

Jin, Hongxiao

Jin, Jiaxin

Jin, Xiaobin

Jolivet, Laurence

Jolliff, Jason K.

Jonah, Olusegun

Jones, Owen

Jones, Penelope J.

Jones, W Linwood

Jonikavičius, Donatas

Jonsson, Bror

Joosse, Pamela J.

Jordan, Tom A.

Jorge, Daniele

José Raimundo, De Souza Passos

José Silva, Maria

Jose, Valdson 
Joshi, Ishan

Joshi, Manish

Joshi, Niraj Prakash

Joshi, Pratik

Jošić, Hrvoje

Jozdani, Shahab Eddin

József, Szatmári

Ju, Mingye

Juan, Carlos G

Juana Sirgado, Luis

Juarez, Eduardo

Jubery, Talukder Z.

Judek, Slawomir

Juez, Carmelo

Jug, Jasmin

Juhasz, Levente

Juhls, Bennet

Jukić, Damir

Julião, Rui Pedro

Julien, Le Kernec

Juliev, Mukhiddin

Jumadi, J.

Jun, Byong-Woon

Jung, András

Jung, Chang Hoon

Jung, Eunsil

Jung, Hahn Chul

Jung, James

Jung, Kichul

Jung, Wook Rak

Junttila, Virpi

Jurado Rodríguez, Juan Manuel

Jurišić, Mladen

Jurjević, Luka

Jurkat, Tina

Jury, Mark

Juszczak, Radoslaw

K. Jain, Sanjay

Kabala, Zbigniew

Kacem, Najib

Kačmařík, Michal

Kaczmarek, Adam L.

Kaczmarek, Halina

Kadela, Marta

Kadhim, Israa

Kadlec, Petr

Kadovic, Ratko

Kaetsch, Christoph

Kafarski, Marcin

Kafatos, Menas

Kafy, Abdulla-Al
Kagone, Stefanie

Kai, Qin

Kaimaris, Dimitris

Kajtoch, Lukasz

Kakareko, Grzegorz

Kakooei, Mohammad

Kaku, Kazuya

Kalaitzidis, Chariton

Kalantar, Bahareh

Kalantari, Mohsen

Kalb, Virginia

Kalbarczyk, Robert

Kalia, Andre

Kalinicheva, Ekaterina

Kalinicheva, Svetlana V.

Kalinnikov, Vladislav

Kalivas, Dionissios

Kall, Tarmo

Kalogeropoulos, Kleomenis

Kalogiros, John

Kaloop, Mosbeh

Kałuża, Tomasz

Kamal Sarker, Mostafa

Kamal, Mohsin

Kamangir, Hamid

Kamh, Samir

Kamini, Yadav

Kamińska, Agnieszka

Kaminska-Chuchmala, Anna

Kamiński, Mirosław

Kamoutsis, Athanasios

Kampel, Milton

Kamusoko, Courage

Kanagaraj, Rajapandian

Kanavos, Andreas

Kandasamy, Sivasathivel

Kandel, Hari

Kane, Van R.

Kanevsky, Mikhail

Kang, Chao

Kang, Emily Lei

Kang, Hyunwoo

Kang, Jeong Min

Kang, Jian

Kang, Jung-Yoon

Kang, Li-Wei

Kang, Mina

Kang, Min-Seok

Kang, Song-Lak

Kang, Xiaoyan

Kang, Yoonja 
Kangro, Kersti

Kanianska, Radoslava

Kantamaneni, Komali

Kantartzis, Nikolaos V.

Kapetanakis, Theodoros N.

Kaplan, Gordana

Kaplan, Gregoriy

Kaplun, Dmitrii

Kapsiotis, Argyrios

Kapuscinski, Tomasz

Karabegovic, Almir

Karagianni, Aikaterini

Karagiannidis, Athanasios

Karaim, Malek

Karakonstantis, Andreas

Karam, Samer

Karantanellis, Efstratios

Karasov, Oleksandr

Karaszewski, Maciek

Karatassiou, Maria

Karatekin, Ozgur

Karathanasi, Flora

Karbasi, Mohammad

Karbou, Fatima

Kargas, George

Kargoll, Boris

Kärhä, Kalle

Karimzadeh, Sadra

Karkani, Anna

Karki, Sita

Karlsson, Karl-Göran

Karna, Yogendra

Karnieli, Arnon

Karoui, Sofiane

Karpov, Ivan V.

Karshakov, Evgeny

Karsznia, Krzysztof R.

Karunaratne, Senani

Karymbalis, Efthimios

Kasampalis, Dimitris

Kašanin-Grubin, Milica

Kaskaoutis, Dimitris

Kasmaee, Sara

Kasperska-Wołowicz, Wiesława

Kasprzak, Włodzimierz

Kasser, Michel

Kästle, Emanuel D.

Kastridis, Aristeidis

Kasymov, Denis

Kasza, Damian

Kataev, Michael Yu
Katagis, Thomas

Katatani, Noritaka

Kateris, Dimitrios

Katila, Matti

Katkovsky, Leonid V.

Kato, Akira

Kato, Seiji

Katona, Krisztián

Katsamakas, Evangelos

Katsanos, Dimitris

Katsantonis, Dimitrios

Katsnelson, Boris

Katsura, Shota

Kaul, Norbert

Kauranne, Tuomo

Kaushik, Prashant

Kavats, Olena

Kaverin, Dmitry A.

Kavianpour, M. R.

Kawai, Yoshimi

Kawakubo, Fernando

Kawalec, Adam

Kawka, Marcin

Kawulok, Michal

Kay, Susan

Kayad, Ahmed Galal

Kayanne, Hajime

Kayano, Mary Toshie

Kayastha, Rijan

Kayetha, Vinay K.

Kayitakire, Francois

Kazak, Jan

Kazakeviciute-Januskeviciene, Giruta

Kazanskiy, Nikolay

Kazimierski, Witold

Kaźmierczak, Rafał

Ke, Xinli

Kechagias-Stamatis, Odysseas

Keckhut, Philippe

Kee, Changdon

Kehagia, Fotini

Keikhosravi Kiany, Mohammad Sadegh

Keim, Corneli

Keith, Darryl

Kelley, Jason

Kelly, Jacque

Kelly, Jeffrey

Kelly, Joshua T.

Kelly, Maggi

Kelly, Natalie

Kelpšaitè-Rimkienè, Loreta 
Kelsey, Eric

Kemp, Jaco

Kempeneers, Pieter

Kenea, Samuel Takele

Kennedy, Ryan

Keppens, Arno

Kereszturi, Akos

Kermarrec, Gaël

Kern, Stefan

Kersten, Tobias

Kertész, Gábor

Keshavarzy, Alireza

Kesik, Jacek

Keski-Saari, Sarita

Keys, Laura

Kganyago, Mahlatse L.

Khadim, Fahad Khan

Khaghani, Farnaz

Khaki, Saeed

Khalaf, Walaa

Khaldi, Rohaifa

Khalefa, Ehsan

Khaleghian, Salman

Khalifa M., Al-Kindi

Khalife, Joe

Khalifeh, Ala

Khamukhin, Alexander

Khan, Ahmad

Khan, Asim

Khan, Faheem

Khan, Ihsan Ullah

Khan, Rizwan Ahmed

Khan, Usman

Khan, Wasiq

Khanal, Uttam

Khand, Kul

Khaniania, Ali Sam

Khare, Siddhartha

Khare, Yogesh

Kharel, Tulsi

Kharismalatri, Hefryan Sukma

Khati, Unmesh

Khatib, Tamer

Khatri, Pradeep

Kheireddine, Malika

Khenchaf, Ali

Kholodkov, Kirill

Khomutov, Artem

Khoramshahi, Ehsan

Khosravi, Khabat

Kiam, Jane Jean
Kiang, Jean-Fu

Kidd, Chris

Kielstra, Brian

Kieu, Hieu Trung

Kikaki, Aikaterini

Kikuchi, Maki

Kikuta, Kazutaka

Kilar, Vojko

Kilic, Lise

Killinger, Dennis K.

Kim, Byung-Gyu

Kim, Chang Ki

Kim, Changwon

Kim, Do-Hyung

Kim, Donguk

Kim, Dukhyeon

Kim, Eonjoo

Kim, EunHee

Kim, Go-Un

Kim, Gunzung

Kim, Hae-Cheol

Kim, Ho Gul

Kim, Hyun Cheol

Kim, Hyunglok

Kim, Jeongeun

Kim, Jeonghwan

Kim, Jeongrae

Kim, Jinwoong

Kim, Jong Suk

Kim, Jonghoek

Kim, Jung-hee

Kim, Jung-Rack

Kim, Kwang Soo

Kim, KyoungSik

Kim, Kyung-Tae

Kim, Namhoon

Kim, Sangdan

Kim, Sangdon

Kim, Seokhyeon

Kim, Seon Jin

Kim, Seong-Cheol

Kim, Seung Hee

Kim, Soontae

Kim, Sungho

Kim, Sung-Min

Kim, Sunmin

Kim, Tae-Jeong

Kim, Wonjun

Kim, Wonkook

Kim, Won-Tae

Kim, Yeseul 
Kim, Yong-Joo

Kim, Yoo-Jun

Kinar, Nicholas J.

Kindu, Mengistie

King, Edward

King, Gregory

King, Owen

King, Stuart

Kingfield, Darrel

Kingon, Kelly

Kinne, Stefan

Kinoshita, Tsuguki

Kinoshita, Yohei

Kinsela, Michael

Kipfstuhl, Sepp

Kirby, Andrew

Kirchoff, Michelle Rosala

Kirichenko, Lyudmyla

Kirillin, Georgiy

Kirillova, Nataliya

Kirk, Randolph

Kishcha, Pavel

Kishi, Naoto

Kiss, Réka

Kiyoyasu, Tanaka

Kjaersgaard, Jeppe

Klämpfl, Florian

Klancar, Gregor

Klapa, Przemysław

Klein, Andrew

Kleindl, William

Klemmt, Hans-Joachim

Kleniewska, Małgorzata

Klimeš, Jan

Klimov, Pavel

Kljusurić, Jasenka Gajdoš

Klobučar, Damir

Klouček, Tomáš

Knaeps, Els

Knapp, Nikolai

Kneen, Melanie Anne

Knioła, Michał

Knoop, Steven

Knowles, James A. C.

Knudby, Anders Jensen

Knyaz, Vladimir

Knyazeva, Maria A.

Ko, Connie

Kobayashi, Hideki

Kobzev, Alexey A.

Kocer, Basaran Bahadir
Kocifaj, Miroslav

Kociu, Arben

Kociuba, Waldemar

Kocourek, Ales

Koc-San, Dilek

Kocur-Bera, Katarzyna

Koda, Kazuhisa

Kodors, Sergejs

Koehler, Philipp

Koelewijn, André Rene

Koerting, Friederike

Koganti, Triven

Kogut, Peter

Kogut, Tomasz

Köhler, Manfred

Kohnová, Silvia

Koike, Yoshikazu

Koirala, Bikram

Koivisto, Satu

Kok, Roeland De

Kokhanovsky, Alexander

Kokin, Osip

Kokinou, Eleni

Kokkalis, Panagiotis

Kokkoris, Ioannis

Kolakowski, Jerzy

Kolb, Andreas

Kolberg, Mariana

Kolerski, Tomasz

Kolios, Stavros

Kolláth, Zoltán

Kolli, Meena Kumari

Kolluru, Venkatesh

Kolokoussis, Pol

Koma, Zsófia

Kómar, Ladislav

Komarek, Jan

Komarkova, Jitka

Komatsu, Teruhisa

Kong, Beibei

Kong, Jian

Kong, Weiping

Kong, Xiangxiong

Kongoli, Cezar

Konik, Marta

Kono, Yasuyuki

Konstantin V., Muzalevskiy

Kontakiotis, George

Kontny, Bernard

Kontoni, Denise-Penelope

Kontopoulos, Ioannis 
Koo, Hongmi

Koo, Younghyun

Kopačková-Strnadová, Veronika

Kopec, Dominik

Kopecká, Monika

Koppa, Akash

Kordi Ghasrodashti, Elham

Koren, Ore

Korhonen, Lauri

Korotaev, G. K.

Korrapati, Hemanth

Korzun, Dmitry

Koschny, Detlef

Kostas, Katselidis

Kostelecký, Jakub

Kostelnick, John

Kostianoy, Andrey G.

Kotchi, Serge-olivier

Kothe, Steffen

Kotlov, Ivan

Kotroni, Vassiliki

Kotthaus, Simone

Kouadio, Louis

Kouba, Jan

Koucká Knížová, Petra

Kouketsu, Takeharu

Koukouli, Maria-Elissavet

Koukouvelas, Ioannis

Kousis, Ioannis

Koutalakis, Paschalis

Koutamanis, Alexander

Koutroumbas, Konstantinos

Koutsia, Nikos

Koutsias, Nikos

Koutsoudis, Anestis

Kouyama, Toru

Kouziokas, Georgios N.

Kováč, Daniel

Kovač-Andrić, Elvira

Kovács, Ferenc

Kovâcs, Péter

Kovács-Győri, Anna

Kovalskyy, Valeriy

Kovanič, Ludovít

Kováŕ, Pavel

Kovarik, Vladimir

Kowalczuk, Piotr

Kowalczyk, Kamil

Kowalewska-Kalkowska, Halina

Kowalewski, Marek

Kozioł, Krystian
Kozlov, Igor E.

Kozlovsky, Alexander E.

Kozubek, Michal

Kpienbaareh, Daniel

Kraft, Marek

Kramm, Tanja

Kranitis, Nektarios

Kranjčić, Nikola

Krasuski, Kamil

Krause, Melanie

Krause, Samantha

Kravtsov, Sergey

Krebes, Edward S.

Kregar, Klemen

Krenke, A. N.

Krenz, Juliane

Kresse, Wolfgang

Krieger, Lukas

Krigsholm, Pauliina

Krinitskiy, Mikhail

Krisanski, Sean

Krishna Moorthy, Sruthi M.

Krishnamurthy, Akshata

Kristian, Yosi

Kritskiy, Dmitriy

Krivá, Zuzana

Krivanek, Vaclav

Križan, Peter

Kroczak, Rafal

Krol, M. C.

Królewicz, Sławomir

Kroszczyński, Krzysztof

Krstic, Djordje

Krtalić, Andrija

Krůček, Martin

Kruczkiewicz, Andrew

Kruk, Edyta

Krynski, Jan

Krzyżaniak, Michał

Kshevetskii, Sergey

Książek, Kamil

Ku, Nian-Wei

Kuang, Wenhui

Kubiak-Wójcicka, Katarzyna

Kubryakov, Alexander

Kubryakov, Arseny

Kucera, Paul

Kucheryavskiy, Sergey

Kuciauskas, Arunas

Kudela, Raphael M.

Kudryavtsev, Vladimir 
Kudrys, Jacek

Kuffer, Monika

Kuhlmann, Gerrit

Kukal, Meetpal

Kul, Khand

Kulawiak, Marek

Kuleshov, Yuriy

Kulpa, Krzysztof

Kumar Batar, Amit

Kumar Singh, Arun

Kumar, Amit

Kumar, Ashutosh Sendil

Kumar, Dipankar

Kumar, K. Raghavendra

Kumar, Kanike Raghavendra

Kumar, Pankaj

Kumar, Rajesh

Kumar, Sanath Sathyachandran

Kumar, Santosh

Kumar, Vinay

Kumar, Vineet

Kumari, Nikul

Kumhála, František

Kumhálová, Jitka

Kuna, Václav

Kundracik, Frantisek

Kuntz, Steffen

Kuo, Chia-Pang

Kuo, Chih-Yu

Kuo, Hsing-Fu

Kuok, Sin-Chi

Kuper, Paul

Kupidura, Przemysław

Kuras, Przemysław

Kurbatova, Juliya

Kurdel, Pavol

Kurdi, Fayez Tarsha

Kurdi, Heba

Kurekin, Andriy

Kurihara, Junichi

Kurihara, Toru

Kuri-Morales, Ángel Fernando

Kuriqi, Alban

Kurkin, Vladimir

Kurkina, Oxana E.

Kuroda, Hiroshi

Kusari, Arpan

Kuschel-Otárola, Mathias

Kusk, Anders

Kussul, Nataliia

Kutnjak, Hrvoje
Kutuza, Boris

Kuze, Hiroaki

Kuželka, Karel

Kuzevicova, Zofia

Kuźma, Marta

Kuzmin, Alexey

Kuznetsov, Sergey

Kwak, Young-Joo

Kwan, Chiman

Kwan-Young, Oh

Kwiatkowski, Tomasz

Kwoczyńska, Bogusława

Kwon, Yonghwan

Kwon, Young-Joo

Kwon, Youngsang

Kwong, Kevin B.

Kycko, Marlena

Kyere, Isaac

Kyparissis, Aris

Kyriakou, Kalliopi

Kyriou, Aggeliki

Kyvelou, Stella Sofia

Kyveryga, Peter

L. Carr, James

L. Wood, Richard

Laamrani, Ahmed

Labant, Slavomir

Labun, Ján

Lacava, Teodosio

Lacaze, Bernard

Lacaze, Roselyne

Lacerenza, Michele

Lacharité, Myriam

Ladstädter, Florian

Laefer, Debra

Laffly, Dominique

Lagain, Anthony

Lagkas, Thomas

Lagostena, Lazaro

Lai, Jhe-Syuan

Lai, Sabrina

Lai, Ying-Chih

Laiolo, Marco

Lajevardipour, Alireza

Lalam, Nageswara

Lalgudi, Hariharan

Lama, Giuseppe Francesco Cesare

Lamb, Brian

Lamberti, Alberto

Lambertini, Alessandro

Lamchin, Munkhnasan 
Lamelas, María Teresa

Lameski, Petre

Lamichhane, Dirga Kumar

Lamichhane, Sushil

Lamine, Salim

Lamparelli, Rubens Augusto Camargo

Lampert, Cory K.

Land, Peter

Landmann, Jannis

Landulfo, Eduardo

Landuyt, Lisa

Lane-Serff, Gregory

Laneve, Giovanni

Lanfredi, Maria

Lang, Haitao

Lang, Mait

Lang, Timothy

Lange, Holger

Lange, Priscila K.

Langheinrich, Maximilian

Lanorte, Antonio

Lantini, Livia

Lanza, Stefania

Lapenna, Vincenzo

Lapucci, Chiara

Lapuerta, Magín

Lara, Carlos

Lara, Luis E.

Lara, Mark Jason

Larisa N., Sidorova

Larose, Eric

Laska, Kamil

Lassalle, Guillaume

Latrubesse, Edgardo

Latte, Nicolas

Latterini, Francesco

Lau, Yui-yip

Laugier, Elise Jakoby

Laukamp, Carsten

Launeau, Patrick

Laureano-Rosario, Abdiel

Laurent, Demagistri

Laurent-Varin, Julien

Laurini, Robert

Lautenbach, Jens

Lauterbach, Helge

Lavrenko, Sergiy

Lavrentiev, Ivan

Lavrova, Olga

Law, Hei

Lawes, Roger A.
Lawson, Adam

Lazar, Michael

Lazaridou, Maria A.

Lázaro, Antonio

Lázaro, Clara

Lazarov, Andon Dimitrov

Lazcano, Raquel

Lazecky, Milan

Lazzari, Maurizio

Le Bris, Arnaud

Le Caillec, Jean-Marc

Le, Anh Tuyen

Le, Manh Hung

Le, Nam Tuan

Leal Gomes, Carlos

Lebart, Ludovic

Lebedev, Sergey

Lebrini, Youssef

Lechner, Alex

Lecigne, Bastien

Lecours, Vincent

Leder, Nenad

Ledwoń, Mateusz

Lee, Bora

Lee, Calvin K.F.

Lee, Changwook

Lee, Chia-Yen

Lee, Chong Hyun

Lee, Chung-Cheng

Lee, Daeyoung

Lee, Danny C.

Lee, Dong-Hoon

Lee, Donghwan

Lee, Dong-Seok

Lee, Eui Chul

Lee, Harry F.

Lee, Il-Gu

Lee, Jae-Kyung

Lee, Jeonghoon

Lee, Jiwan

Lee, Jonghun

Lee, Joonho

Lee, Joonwhoan

Lee, Ju

Lee, Jun Hak

Lee, Keunhwa

Lee, Kiwon

Lee, Kwang-Jae

Lee, Meonghun

Lee, Min-sun

Lee, Moonjin 
Lee, Moon-Kyu

Lee, Moungjin

Lee, Sang Heon

Lee, Sang Jun

Lee, Sanggyun

Lee, Sang-Hyun

Lee, Sang-Moo

Lee, Sangwoo

Lee, Se-Jin

Lee, Seongwook

Lee, Seo-Young

Lee, Sukho

Lee, Sunmin

Lee, Taesam

Lee, Woo-Kyung

Lee, Yang-Won

Lee, Yo-Han

Lee, Yun Gon

Lees, Kirsten

Leger, Jean-Michel

Legleiter, Carl

Lehmann, Moritz

Lehner, Arthur

Lehnert, Michal

Lehr, Bill

Lehtola, Ville

Lei, Guangchun

Lei, Manchun

Lei, Ruibo

Lei, Tao

Lei, Wentai

Lei, Yanbin

Leibovici, Didier G.

Lein, James

Leitch, Kenneth

Leite, Helio Garcia

Lejba, Paweł

Lekic, Nikola

Lemmens, Mathias

Lemnaru, Camelia

Lemos Cid, Edgar

Lemus-Canovas, Marc

Leń, Przemysław

Leng, Pei

Lenz, Dominik

Leoidivna, Sergieieva Kateryna

Leon, Iñigo

Léon, Jean-Francois

Leon, Jose Raul Romo

Leonardi, Mauro

Leonardini, Gonzalo
Leone, Antonio Pasquale

Leone, Natalia

Leon-Tavares, Jonathan

Lepoglavec, Kruno

Lerones, Pedro Martín

Lerot, Christophe

Leśny, Jacek

Lessire, Françoise

Leucci, Giovanni

Levashova, Natalia

Levesque, Juan C.

Levin, Eugene

Levin, Noam

Levine, Judah

Levis, Aviad

Levitan, Nathaniel

Levy, Clara

Levy, Gad

Levy, Ilan

Levy, Robert

Lewandowicz, Elżbieta

Lewińska, Paulina

Lewiński, Stanisław

Lewis, Quinn

Lewis, Tom

Lewis, William E.

Leyser, Thomas B.

Lezzerini, Marco

Lhissou, Rachid

Lhotáková, Zuzana

Li, Bo

Li, Chunlin

Li, Congcong

Li, Dengqiu

Li, Fashuai

Li, Fei

Li, Feng

Li, Guiying

Li, Haifeng

Li, Haiyan

Li, Hanhui

Li, Hao

Li, Hongyi

Li, Huan

Li, Hui

Li, Jiada

Li, Jialin

Li, Jianping

Li, Jiayi

Li, Jin

Li, Jiwei 


\begin{tabular}{|c|c|}
\hline Li, Jiyu & Li, Yue \\
\hline Li, Jun & Li, Yuxing \\
\hline Li, Junli & Li, Zhan \\
\hline Li, Kaitao & Li, Zhaoguo \\
\hline Li, Lanhai & Li, Zhe \\
\hline Li, Leilei & Li, Zhenyu \\
\hline Li, LiHaojun & Li, Zhijia \\
\hline Li, Lin & Li, Zhilin \\
\hline Li, Linchao & Li, Zhiwei \\
\hline Li, Lingling & Li, Zhixiong \\
\hline Li, Lingqiao & Li, Zhouyuan \\
\hline Li, Liwei & Li, Zuchuan \\
\hline Li, Long & Lia, Marchi \\
\hline Li, Meifang & Liang, Binqing \\
\hline Li, Mengmeng & Liang, Boyi \\
\hline Li, Meng-Syue & Liang, Cunren \\
\hline Li, Min & Liang, Hongyu \\
\hline Li, Mingshi & Liang, Jian \\
\hline Li, Ning & Liang, Liang \\
\hline Li, Pan & Liang, Tiangang \\
\hline Li, Peng & Liang, Wanwan \\
\hline Li, Qingting & Liang, Zhongyao \\
\hline Li, Qingyu & Liangke, Huang \\
\hline Li, Rui & Liao, Chunhua \\
\hline Li, Shengyang & Liao, Enhui \\
\hline Li, Shuang & Liao, Jingjuan \\
\hline Li, Siwei & Liao, Mi \\
\hline Li, Tiancheng & Liao, Tien-Hao \\
\hline Li, Wang & Liao, Xiaohan \\
\hline Li, Wei & Liaw, Shyue-Cherng \\
\hline Li, Weiqiang & Libra, Martin \\
\hline Li, Wenjuan & Lidón, Antonio \\
\hline Li, Wenliang & Liesenberg, Veraldo \\
\hline $\mathrm{Li}, \mathrm{Xi}$ & Ligaray, Mayzonee \\
\hline Li, Xianqi & Ligi, Martin \\
\hline Li, Xiaobo & Lilin, Zhang \\
\hline Li, Xiaodong & Lillo-Saavedra, Mario \\
\hline Li, Xiaoming & Lim, Deok Won \\
\hline Li, Xiaopeng & Lim, Ducsun \\
\hline Li, Xiaorun & Lim, Gilbert \\
\hline Li, Xing & Lim, Gyu-Ho \\
\hline Li, Xingdong & Lim, Kyosun \\
\hline Li, Xinghua & Lim, Nancy Joy \\
\hline Li, Xiuhong & Lim, Sohee \\
\hline Li, Xuecao & Limongelli, Maria Giuseppina \\
\hline Li, Xungui & Limongiello, Marco \\
\hline Li, Yanan & Limp, W. Fred \\
\hline Li, Yansheng & Lin, Chen \\
\hline Li, Yao & Lin, Chien-Chou \\
\hline Li, Yuanhao & Lin, Chin-Feng \\
\hline Li, Yubin & Lin, Daw-Tung \\
\hline
\end{tabular}


Lin, Guoqing

Lin, Hanzhi

Lin, Huei-Yung

Lin, Huilong

Lin, Jenshan

Lin, Jianmin

Lin, Jianzhe

Lin, Jiayuan

Lin, $\mathrm{Li}$

Lin, Lili

Lin, Lin

Lin, Pay Liam

Lin, Shangrong

Lin, Tang-Huang

Lin, Teng-Chiu

Lin, Wen-Yinn

Lin, Ya-Ping

Lin, Ying-Tien

Lin, Yu-Ching

Lin, Yuewei

Lin, Zihan

Linck, Roland

Lind, Brianna M.

Lindberg, Eva

Ling, Feng

Ling, Pui-Yu

Linklater, Michelle

Linscott, Ivan R.

Lio, Giuseppe Emanuele

Liou, Yuei-An

Lipa, Belinda

Lippe, Melvin

Lipuš, Bogdan

Lira, Cristina

Liran, Oded

Liritzis, Ioannis

Lisetskii, Fedor

Lisowski, Józef

Lister, Andrew

Litkey, Paula

Lityński, Piotr

Liu, Bin

Liu, Binxin

Liu, Bo

Liu, Caixia

Liu, Changjun

Liu, Chao

Liu, Chunwei

Liu, Dong

Liu, Fei

Liu, Feifeng
Liu, Guoxiang

Liu, Hai

Liu, Hailong

Liu, Hao

Liu, Hongfu

Liu, Hua

Liu, Huizeng

Liu, Jiahong

Liu, Jialin

Liu, Jiangang

Liu, Jianhong

Liu, Jianhua

Liu, Junmin

Liu, Kai

Liu, Keng-Hao

Liu, Liangyun

Liu, Linshan

Liu, Lintao

Liu, Meiling

Liu, Minliang

Liu, Ning

Liu, Peng

Liu, Po

Liu, Qi

Liu, Qian

Liu, Qiang

Liu, Quanhua

Liu, Rong

Liu, Ronggao

Liu, Saiyan

Liu, Shanjun

Liu, Shaomin

Liu, Shaoqing

Liu, Shufan

Liu, Shu-Zhi

Liu, SiCong

Liu, Suxing

Liu, Tao

Liu, Wai Chung

Liu, Weibo

Liu, Wei-Min

Liu, Wen

Liu, Wen-Cheng

Liu, Xiang

Liu, Xianglin

Liu, Xiangzhuo

Liu, Xiaoming

Liu, Xing

Liu, Xingpeng

Liu, Yan

Liu, Yan-An 
Liu, Yang

Liu, Yaqun

Liu, Yi

Liu, Yonggang

Liu, Yuanyuan

Liu, Yubao

Liu, Zhang-Meng

Liu, Zhe

Liu, Zheng

Liu, Zhengjia

Liu, Zhenqing

Liu, Zhifeng

Liu, Zhifu

Liu, Zhiyu

Liu, Zhong

Liu, Zhuo

Liuzzi, Giuliano

Livens, Stefan

Livieris, Ioannis E.

Livio, Franz A.

Lizarralde, Iñigo

Lizurek, Grzegorz

Ljubenovic, Marina

Llaveria Godoy, David

Llovería, Raquel Montorio

Lo Brutto, Mauro

Lo Feudo, Teresa

Lo Giudice, Angelina

Lo, Kwong Fai Andrew

Lo, Shi-Wei

Lo, Yueh-Hsin

Loaiciga, Hugo

Loaiza Cerón, Wilmar

Lobell, David

Lobiuc, Andrei

Lobo, Agustin

Löcher, Anno

Loddo, Andrea

Logan, Timothy

Loghin, Ana Maria

Loh, Andrew

Loiko, Sergey

Lok Woo, Wai

Lolli, Simone

Lombardi, Federico

Lombardi, Jason

Long, Tengfei

Long, Xin

Longchamps, Louis

Longépé, Nicolas

Lopes Rosa, Renata
Lopes, Fernand

Lopes, Francis M.

Lopes, José Fortes

Lopes, Waslon

López González, Alejandro

Lopez, Jaime

López, Josué

Lopez-Baeza, Ernesto

Lopez-Cuervo Medina, Serafin

López-Granados, Francisca

Lopez-Molina, Carlos

López-Serrano, Pablito M.

Loprencipe, Giuseppe

Lord, Etienne

Lorente, Pablo

Łoś, Helena

Lottering, Romano

Lou, Hezhen

Lou, Yidong

Louis, Jérôme

Louka, Panagiota

Loupasakis, Constantinos

Loures, Luis

Lovas, Tamás

Lovergine, Francesco Paolo

Lovino, Miguel

Lovisolo, Lisandro

Lowe, Roger C.

Lowell, Kim

Löytönen, Markku

Lozano Baez, Sergio Esteban

Lozano Parra, Javier

$\mathrm{Lu}, \mathrm{Biao}$

Lu, Bing

$\mathrm{Lu}$, Chih-Heng

Lu, Ching-Ta

Lu, Gaopeng

$\mathrm{Lu}$, Heng

Lu, Lei

$\mathrm{Lu}$, Linlin

Lu, Wenfang

$\mathrm{Lu}, \mathrm{Xiao}$

Lu, Xiaoman

$\mathrm{Lu}$, Xiaomei

Lu, Yan

Lubac, Bertrand

Lubczonek, Jacek

Lucà, Federica

Lucas, Benjamin

Lucas, Christopher

Lucas, Steven 
Lucchesi, David

Lucchi, Elena

Lucille, Alonso

Lück-Vogel, Melanie

Lüdecke, Horst-Joachim

Ludwig, Valentin

Luhar, Ashok

Lui, Antonny

Lui, Hoi-Shun

Luiz, Alfredo José Barreto

Luján-Álvarez, Concepcion

Lukac, Martin

Lukač, Niko

Lukáš, Jan

Lukasová, Veronika

Lukin, Vladimir

Łukowski, Mateusz

Lumban-Gaol, Jonson

Lundgren, Paul R.

Luning, Sebastian

Lunkov, Andrey

Luo, Bin

Luo, Bingkun

Luo, Dongliang

Luo, Fa-Long

Luo, Fulin

Luo, Huiying

Luo, Jia

Luo, Jiali

Luo, Lei

Luo, Lihui

Luo, Pingping

Luo, Tao

Luo, Yi

Luo, Ying

Lupiano, Valeria

Lupidi, Alberto

Luppichini, Marco

Lupu, Lucian

Lupyan, Evgeny

Luque-Nieto, Miguel Angel

Lurz, Fabian

Lušić Vukić, Darija

Lutnesky, Marvin M. F.

Lv, Mingyang

Lyakhov, Andrey

Lykhovyd, Pavlo

Lynch, Mervyn

Lynn, Barry $\mathrm{H}$.

Lyons, Ashley

Lyu, Fanchao
Lyu, Hong-Kun

Lyubushin, Alexey

Lyulyukin, Vasily S.

Lyzenga, David

Ma, Andong

Ma, Chunfeng

Ma, Chunyong

Ma, Dan

Ma, Der-Ming

Ma, Ding

Ma, Dongdong

Ma, Fei

Ma, Guanyi

Ma, Hongchao

Ma, Hongyang

Ma, Hui

Ma, Jun

Ma, Lei

Ma, Lei-Ming

Ma, Lingling

$\mathrm{Ma}$, Mingguo

Ma, Qin

Ma, Weiqiang

$\mathrm{Ma}, \mathrm{Wu}$

$\mathrm{Ma}$, Xiaofei

Ma, Xiaoshuang

Ma, Yaoming

Ma, Yingying

Ma, Yingzhao

Ma, Yujun

Ma, Zhenling

Maalek, Reza

Maccari, Attilio

Macedonio, Giovanni

Mach, Douglas M.

Machado, Elia A.

Machado, Nadja

Machado, Renato

Machaj, Juraj

Machowski, Witold

Machwitz, Miriam

Mackovjak, Simon

Macků, Karel

Madero Ayora, María José

Madewell, Charles

Madhavan, Bomidi Lakshmi

Madhavan, Sriharsha

Madokoro, Hirokazu

Madonia, Alice

Madonsela, Sabelo

Madra, Anna 
Madroñal, Daniel

Madson, Austin

Madurapperuma, Buddhika

Maeda, Jun

Maesano, Mauro

Magalhães, Luís Gonzaga

Magalhães, Paulo Sérgio Graziano

Magdy, Hatem

Maggioni, Viviana

Maghsood, Fadia Fatemeh

Magidi, James

Magliulo, Paolo

Magno, Ramona

Magnuszewski, Artur

Mahajan, Gopal Ramdas

Mahajan, Sachit

Mahan, J. Robert

Mahdavipour, Omid

Mahdianpari, Masoud

Mahdy, Amr Mohamed Samy

Maheng, Dikman

Mahmoodi, Ali

Mahmoud Marghany, Maged

Mahmud, M. Rizaludin

Mahmud, Sultan

Mahoney, Craig

Mahoney, Robert

Maia, Dalmiro

Maia, Rodrigo Filev

Maidment, Ross

Maietti, Federica

Maimaitijiang, Maitiniyazi

Maimaitiyiming, Maimaiti

Maimaitiyiming, Matthew

Main, Russell

Maingi, John

Maiwald, Ferdinand

Majdański, Mariusz

Majeed, Abdul

Majeed, Yaqoob

Majidi Nezhad, Meysam

Majurec, Ninoslav

Mak, Hugo Wai Leung

Makantasis, Konstantinos

Makar, Artur

Maki, Masayasu

Makinde, Esther O.

Maksymilian, Solarski

Maktabi, Marianne

Mäkynen, Marko

Malambo, Lonesome
Maleska, Tomasz

Malihi, Shirin

Malik, Amir Haider

Malik, Ida Bagus Ilham

Malik, Mashkoor

Malings, Carl

Malinowska, Agnieszka

Malinowski, Radek

Małka, Anna

Mallast, Ulf

Mallick, Javed

Mallinis, Giorgos

Malone, Andrew

Malos, Cristian

Maltezos, Evangelos

Maltseva, O. A.

Maltseva, Olga

Malvić, Tomislav

Mancinelli, Enrico

Mancinelli, Paolo

Mancini, Adriano

Mandanici, Emanuele

Mandarino, Andrea

Manders, Astrid

Mandlburger, Gottfried

Manea, Marina

Manfreda, Salvatore

Manfredi, Giovanni

Mangina, Eleni

Mangold, Alexander

Mangold, Mikael

Manivasagam, V.S.

Manoharan, Prabukumar

Mansi, Ahmed Hamdi

Mansilha, Catarina

Mansour, Shawky

Mantas, Vasco

Manton, Michael

Mantovani, Matteo

Manucharyan, Georgy E.

Manzano-Sarabia, Marlenne

Manzu, Ciprian Claudiu

Mao, Chongyuan

Mao, Dehua

Mao, Jiafu

Maradin, Mladen

Marcato Júnior, José

Marceta, Dusan

Marche, Claudio

Marcheggiani, Ernesto

Marchel, Łukasz 
Marchelli, Maddalena

Marchesini, Luca Belelli

Marchetti, Dedalo

Marchetti, Francesca

Marchevský, Stanislav

Marchisio, Alberto

Marcolin, Enrico

Marconi, Sergio

Marengo, Jose A.

Mares, Jan

Mărgărint, Mihai Ciprian

Margvelashvili, Nugzar

Marian, Dacian-Paul

Marin, Carlo

Marin, Roberto J.

Marinangeli, Lucia

Marinelli, Daniele

Marinello, Francesco

Marin-Hernandez, Antonio

Marino, Eva

Marino, Pasquale

Marinos, Vassilis

Mario, Muštra

Mariotti, Mauro

Markos, Nikos

Markovic, Goran B

Marković, Milena

Markovinović, Danko

Marks, David A.

Markušić, Snježana

Markuszewski, Piotr

Marmol, Urszula

Marmorino, George O.

Marpu, Prashanth Reddy

Marquardt, Christian

Marques Ramos, Ana Paula

Marques, Gonçalo

Marques, Haroldo

Marquis, Jared

Marren, Philip M.

Mars, John C.

Marsh, Stuart

Marshall, Gareth

Marsico, Antonella

Martel, Ernestina

Martellucci, Riccardo

Martian, Alexandru

Martín Del Campo, Gustavo D.

Martin, Angel

Martin, Daniel F.

Martin, Iain
Martin, Julien

Martin, Laura Gomez

Martin, Maria

Martin, Maxence

Martin, Tara J.

Martinaitis, Steven

Martín-Crespo, Tomás

Martinelli, Giovanni

Martinelli, Massimo

Martin-Escalona, Israel

Martínez Vázquez, Maria Luisa

Martinez, Beatriz

Martínez-Carricondo, Patricio

Martinez-Izquierdo, Estibaliz

Martínez-Marín, Tomas

Martínez-Otzeta, José María

Martin-Herrero, Julio

Martin-Neira, Manuel

Martino, Gerardo Di

Martino, Luca

Martín-Ortega, Pablo

Martín-Porqueras, Fernando

Martins De Assis, Carlos André

Martins, Ana

Martins, Fernando Ramos

Martins, João P. A.

Martins, Marcos

Martins, Meike Sena

Martins, Rui Costa

Martone, Michele

Martucci, Matteo

Marullo, Salvatore

Maruyama, Yoshihisa

Mas Caussel, Jean-François

Mäs, Stephan

Masar, Matej

Mascarenhas, Nelson D. A.

Mascioli, Francesco

Mascitelli, Alessandra

Mascolo, Lucio

Mascort-Albea, Emilio J.

Mase, Atsushi

Maset, Eleonora

Mashimbye, Zama Eric

Masiero, Andrea

Maskell, Lindsay C.

Maslakov, Alexey

Masný, Matej

Mason, Robert

Masoumi, Salim

Masri, Bassil El 
Masrur, Arif

Massarelli, Carmine

Masse, Antoine

Massoud, Elias

Mastbergen, Dick R.

Mastelić Ivić, Siniša

Mastorakis, Nicos

Mastrogiuseppe, Marco

Masud, M Badrul

Mata-Lima, Herlander

Matamoros, David

Matano, Fabio

Matasaru, Petre Daniel

Matasović, Brunislav

Mataveli, Guilherme

Mataveli, Guilherme Augusto Verola

Maté González, Miguel Ángel

Matejka, Karel

Materazzi, Marco

Mateus, Pedro

Mather, Ben

Mathew, Joice

Matias, Márcia

Matic, Frano

Matiu, Michael

Matlovičová, Kvetoslava

Matricardi, Marco

Matricciani, Emilio

Matrone, Francesca

Matsubara, Edson Takashi

Matsuo, Hiroshi

Matsushima, Dai

Mattar, Mohamed

Matthews, Grant

Matthews, Jessica

Mattias, Gaglio

Mattingly, Kyle

Mattioli, Vinia

Maturi, Eileen

Matuz, Balazs

Matzler, Christian

Matzner, Shari

Maugis, Pascal

Mauriello, Paolo

Mauro, Giorgio Sebastiano

Mauro, Giovanni

Mauser, Wolfram

Mavraeidopoulos, Athanasios K.

Mavromatis, Theodoros

Mayer, Larry

Mayer, Winfried
Maynard, Jonathan J.

Mayoral Herrera, Victorino

Mayos, Lluís Pesquer

Mayra Alejandra, Roman-Rivera

Mazabraud, Yves

Mazurek, Przemysław

Mazza, Alessandro

Mazzei, Mauro

Mazzetti, Paolo

Mazzoleni, Maurizio

Mboga, Nicholus O.

McCallum, Adrian

McCallum, Lucia

McCarter, Michael K.

McClenachan, Giovanna

McClinton, J. Timothy

McCoy, Mark D.

McDonald, Adrian

McGaughey, Robert

Mcgill, Matthew J.

McGovern, Eugene

McGrath, Ciara N.

McGrath, Heather

McGuire, Michael

McGuire, Patrick

McKay, Patricia

McKenna, Phillip

McKerrow, Alexa

McNabb, Robert W

Mcneill, Stephen

McRoberts, Ronald E.

Medeiros, Stephen

Medic, Tomislav

Medić, Tomislav

Medina, Daniel

Medyńska-Gulij, Beata

Meggio, Franco

Mehan, Sushant

Mehmood, Rashid

Mei, Alessandro

Mei, Liang

Mei, Xiaoguang

Meier, Cecile

Meier, Watlter

Meijer, Yasjka

Meisen, Tobias

Meisina, Claudia

Meister, Gerhard

Mejia Aguilar, Abraham

Mejias, Luis

Mekhalfi, Mohamed Lamine 
Mel, Riccardo

Melani, Samantha

Melendez-Pastor, Ignacio

Melillos, George

Mello, Carlos Rogério

Mellouli, Nédra

Melnichenko, Oleg

Melo, Pedro

Meloni, Fernando

Melo-Pinto, Pedro

Mendes, Mateus

Mendes, Renato

Mendes, Virgilio Brito

Mendes, Wanderson De Sousa

Mendez-Barroso, Luis

Mendez-Vazquez, Andres

Mendoza-Fernández, Antonio

Menegatti, Carlos Renato

Meneghini, Robert

Menéndez, Héctor D.

Meng, Hongying

Meng, Qingkai

Meng, Qingmin

Meng, Qingyan

Meng, Ran

Meng, Xingmin

Meng, Yuan

Meng, Zhiguo

Menn, Marc Le

Mentasti, Simone

Menzel, Christopher M.

Menzies, Robert T.

Meo, Marianna

Meoni, Gabriele

Mercado, Jesús M.

Mercatoris, Benoit

Merchan, Carlos Iglesias

Merchán, Pilar

Merciol, François

Merlin, Olivier

Mermoz, Stéphane

Meron, Moshe

Meroño, Jose Emilio

Merrick, Trina

Mertikas, Stelios

Mertzimekis, Theo

Merwe, Deon Van Der

Mesa, Oscar

Mesa-Mingorance, José Luis

Mesas Carrascosa, Francisco Javier

Meschini, Alessandra
Meshesha, Tesfa Worku

Messina, Gaetano

Messina, Janey

Mestas-Nuñez, Alberto M.

Mezei, Pavel

Miah, Md Sipon

Miano, Andrea

Miao, Ruiqing

Miccadei, Enrico

Miccinesi, Lapo

Michaelides, Roger

Michaelsen, Eckart

Michalak, Anna

Michalak, Dariusz

Michalopoulou, Zoi-Heleni

Michalowska, Joanna

Michel, Aurélie

Michel, Julien

Micheli, Carla

Michelini, Alberto

Michetti, Alessandro

Michler, Oliver

Micijevic, Esad

Mickler, Robert A.

Mielewczik, Michael

Mielke, Christian

Mielonen, Tero

Miettinen, Jukka

Migała, Krzysztof

Migallón, Héctor

Migliaccio, Maurizio

Mihai, Bogdan Andrei

Mihai, Ilie Valentin

Mihai, Laura

Mihajlović, Dragan

Mijić, Zoran

Mikesell, Thomas Dylan

Mikheeva, Anna

Mikita, Tomáš

Milan, David

Milani, Lisa

Milelli, Massimo

Milenković, Milan

Milenov, Pavel

Miler, Mario

Milillo, Pietro

Milinevsky, Gennadi

Militino, Ana Fernández

Miljković, Predrag

Millan Almaraz, Jesus Roberto

Millar, Phillip 
Millefiori, Leonardo M.

Miller, Boris

Miller, Douglas

Miller, Gwen J.

Miller, Jarrod O.

Miller, John H.

Milosavljević, Aleksandar

Mimouna, Amira

Min, Xia

Minařík, Robert

Minářová, Jana

Mind'áš, Jozef

Minea, Ionut

Minelli, Annalisa

Minelli, Liliana

Minematsu, Tsubasa

Minetto, Alex

Ming, Dongping

Minjarez Sosa, Carlos Manuel

Minkyung, Son

Minotti, Priscilla

Minschwaner, Kenneth R.

Mirabella Roberti, Giulio

Miranda, Maria D.

Miranda, Pedro M. A.

Mirhashemi, Arash

Miřijovský, Jakub

Mirjalili, Seyedali

Mirmazloumi, Mohammad

Miron, Alina

Miroshnichenko, Leonty

Mishne, David

Mishra, Amit

Mishra, Amitabh

Mishra, Bhogendra

Mishra, Deependra

Mishra, Sachidananda

Misra, Gourav

Misra, Sidharth

Misra, Vasu

Mistretta, Fausto

Misumi, Ryohei

Mitasova, Helena

Mitchell, David L.

Mitchell, Scott

Mitić, Petar

Mitka, Bartosz

Mittempergher, Silvia

Miura, Hiroyuki

Miyama, Toru

Miyata, Takamichi
Miyazaki, Hiroyuki

Miyazaki, Tomo

Miyazawa, Yasumasa

Mizuochi, Hiroki

Mizutani, Norimi

Mleczko, Magdalena

Mlekuž, Dimitrij

Moafipoor, Shahram

Mobasheri, Amin

Mocnik, Franz-Benjamin

Modala, Naga Raghuveer

Modica, Giuseppe

Moeller, Chris

Moeslund, Jesper Erenskjold

Mohajane, Meriame

Mohajerani, Sorour

Mohamed, Bayoumy

Mohamed, Hassan

Mohammadi, Babak

Mohammadi, Masoud

Mohammadi, Mohammad Ebrahim

Mohammadloo, Tannaz $\mathrm{H}$.

Mohammadzadeh, Ali

Mohammed, Ahmed

Mohammed, Ali

Mohapatra, Sarat

Mok, Sung-Hoon

Molero, Francisco

Molina, Herminia

Molina, Inigo

Molina, Juan Ramón

Molina, Rafael

Molina-Martínez, José Miguel

Molinier, Matthieu

Molkov, Alexander

Mollalo, Abolfazl

Møller, Anders Bjørn

Möller, Gregor

Molowny-Horas, Roberto

Molto, Enrique

Momm, Henrique

Mon, Yi-Jen

Mona, Lucia

Monahan, Edward C.

Monchetti, Silvia

Mondal, Dhiman

Mondini, Alessandro C.

Mondino, Enrico Corrado Borgogno

Mondol, Anarul Haque

Mongus, Domen

Monico, Joao Francisco Galera 
Monsivais-Huertero, Alejandro

Montagnani, Leonardo

Montalvan-Burbano, Nestor

Monteiro Gonçalves, José Manuel

Monteiro Santos, Fernando

Monteiro-Henriques, Tiago

Monterroso-Checa, Antonio

Montes, Hector

Montes, Marcos

Montgomery, Flora

Montgomery, Jack

Monti Guarnieri, Andrea Virgilio

Montillet, Jean-Philippe

Montisci, Augusto

Montopoli, Mario

Montull, Jose M.

Moon, Il-Ju

Moon, Jihoon

Moon, Minkyu

Moon, Todd

Moore, Nathan

Moore, Philip

Mora Kuplich, Tatiana

Mora, Andre

Morabito, Anna

Morabito, Marco

Morales, Carlos

Morales, Luis

Morales-Caselles, Carmen

Morar, Cezar

Moraru, Paula Ioana

Moras, Julien

Morbidoni, Christian

Mordret, Aurélien

Moreira, Joao

Moreira, Lorena

Morel, Julien

Moreno, Adam

Moreno, Jose

Moreno, Sergio

Moreno-Armendáriz, Marco A.

Moreno-Madrinan, Max

Moretti, Sandro

Morgan, G. A.

Morgan, Grayson

Morgans, Courtney

Mori, Emiliano

Morimoto, Takeshi

Moroni, Davide

Morović, Mira

Morozov, Evgeny
Morozova, Anna

Morris, Daniel

Morris, Mary

Morsy, Salem

Morton, Daniel

Mosavi, Amir

Mosca, Nicola

Moshe, Boaz Ben

Moshou, Alexandra

Mösinger, Leander

Moskalik, Tadeusz

Mota, Guilherme Lucio Abelha

Motagh, Mahdi

Motahhir, Saad

Mott, Rowan

Motteler, Howard

Mou, Lichao

Moudry, Vítezslav

Mount, Andrew

Mouratidis, Antonios

Mousavi, Amirhosein

Moussa, Mohamed

Moussas, Vassilios C.

Moyano Campos, Juan José

Moyer, Jonathan D.

Mozgeris, Gintautas

Mozos, Daniel

Mozzi, Paolo

Mrak, Sebastijan

Mráz, Alexander

Mrocheń, Dawid

Mrokowska, Magdalena

Mrówczyńska, Maria

Mróz, Marek

$\mathrm{Mu}$, Mingquan

Mueller, Rick

Mughees, Atif

Mugiraneza, Theodomir

Muhuri, Arnab

Mukherjee, Anandarup

Mukherjee, Kriti

Mukherjee, Lipi

Mukherjee, Mithun

Mukherjee, Subhayan

Mulahusić, Admir

Mulas, Marco

Mulianga, Betty

Mullen, Linda

Muller, Andrew C.

Müller, Hartmut

Mulualem, Getachew Mehabie 
Mulverhill, Chris

Munasinghe, Dinuke

Munawar, Sana

Muncan, Jelena

Münch, Zahn

Mund, Jan-Peter

Munghemezulu, Cilence

Munini, Riccardo

Munir, Md. Shirajum

Munir, Tariq M.

Muñoz, Juan Jose

Muñoz-Marí, Jordi

Munoz-Martin, Joan Francesc

Munoz-Pacheco, Jesus M.

Muntoni, Giacomo

Murakami, Hiroshi

Muralikrishnan, Bala

Muramatsu, Kanako

Murárescu, Ovidiu-Marcel

Murashkin, Dmitrii

Murayama, Yuji

Murböck, Michael

Muresan, Mircea Paul

Murray, Jonathan

Murtha, Timothy M.

Murtiyoso, Arnadi

Murugan, Deepak

Murzin, Anton

Musacchio, Massimo

Musayev, Sardorbek

Muskett, Reginald R.

Mustafa, Ahmed

Mustapha, Meftah

Mustaqeem, Mustaqeem

Muszyński, Zbigniew

Mutowo, Godfrey

Muttin, Frédéric

Muzirafuti, Anselme

Muzylev, Eugene

Myakalwar, Ashwin Kumar

Myeong, Seunghwan

Myeong, Soojeong

Myers, Paul

Myoung, Boksoon

Myroniuk, Viktor

Myrovali, Glykeria

Myslenkov, Stanislav

Mzid, Nada

$\mathrm{Na}$, Jiaming

Naaji, Antoanela

Nabavi, Seyed Omid
Nabil, Bachagha

Nabil, Mohsen

Naccarato, Kleber Pinheiro

Nacheva, Radka

Nadal-Sala, Daniel

Nadir Arslan, Ali

Nadolny, Adam

Naga, Coulibaly

Nagai, Hiroto

Nagano, Hirohiko

Nagaoka, Hiroshi

Naghshvarianjahromi, Mahdi

Nagirner, Dmitrij I.

Nagisetty, Raja

Nagle-McNaughton, Timothy

Nagol, Jyoteshwar R.

Nagy, Balázs

Nagy, Gustavo

Nagy, Hazem

Naidoo, Laven

Naik, Sambit Prasanajit

Najibi, Nasser

Nakalembe, Catherine

Nakamichi, Haruhisa

Nakamura, Kenji

Nakamura, Ryota

Nakamura, Tomoaki

Nakata, Makiko

Nakazawa, Yoshinori

Nalej, Marta

Nalepa, Jakub

Nalewajko, Joanna Sekulska

Nam, Dinh Van

Nam, Kwang Woo

Nam, Soonkie

Nam, SungHyun

Nanding, Nergui

Nanni, Loris

Nanni, Marcos

Nannipieri, Pietro

Naokazu, Taniguchi

Napoli, Mariano Di

Nappi, Rosa

Naqvi, RizwanAli

Naraei, Parisa

Näränen, Jyri

Naranjo, José Manuel

Narapusetty, Balachandrudu

Narayanan, Ram M.

Narayanan, RM

Nardi, Fernando 
Nardini, Andrea

Nardino, Marianna

Nardino, Vanni

Nardone, Gabriele

Narine, Lana Landra

Narouei-Khandan, Hossein A.

Nasahara, Kenlo

Nasahara, Kenlo Nishida

Nascimento, Daniel Chagas Do

Naser, M. Z.

Nashwan, Mohamed Salem

Nasonova, Sasha

$\mathrm{Naß}$, Andrea

Nassar, Ayman

Nastase, Eduard Ilie

Nastula, Jolanta

Natalia, Kablak

Nate, Silviu

Natsuaki, Ryo

Naumov, Vitalii

Naus, Krzysztof

Nava-Baro, Enrique

Navarro, Andrés

Navarro, Omar Gutierrez

Navarro, Victor

Navarro-Pedreño, Jose

Navas-Carrillo, Daniel

Navrátil, Václav

Nayak, Sridhara

Nazarkevych, Mariia

Nazeer, Majid

Ndayisaba, Felix

Nduati, Eunice

Neagoe, Mircea

Nechaev, Dmitri

Nechita, Constantin

Nee, Jan B.

Negm, Abdelazim

Negru, Mihai

Negusini, Monia

Neish, Andrew

Nejedlik, Pavol

Nekaris, Anne

Nelson, Jake

Nemeth, Karoly

Nemuc, Anca

Nepal, Ramesh

Neri, Marco

Nertan, Argentina

Nesje, Atle

Netzel, Pawel
Neudegg, David

Neumann, Carsten

Neumann, Mathias

Neumann, Wiebke

Neuville, Romain

Nevalainen, Paavo

Neves, Alana Kasahara

Neves, José

Newlands, Nathaniel K.

Nezami, Somayeh

$\mathrm{Ng}$, Alfred

$\mathrm{Ng}$, Brian

$\mathrm{Ng}$, Ding-Quan

Ngo, Dat

Ngoy, Kikombo

Nguyen, Giao

Nguyen, Huong Thu

Nguyen, Khuong An

Nguyen, Kim Anh

Nguyen, Lan

Nguyen, Lan Hoang

Nguyen, Thanh Huy

$\mathrm{Ni}$, Chuen-Fa

$\mathrm{Ni}$, Huan

$\mathrm{Ni}$, Jun

Niacsu, Lilian

Nichols, C. Reid

Nickels, Kevin

Nicodemo, Gianfranco

Nicola, Masini

$\mathrm{Nicu}$, Ionut Cristi

Niculescu, Simona

Niculita, Mihai

Nie, Tiezheng

Niedbała, Gniewko

Niemann, Olaf

Niemeier, Wolfgang

Nieto Julián, Juan Enrique

Nieves, Jeremiah

Niki-Maleki, Khashayar

Nikitin, Nikolay O.

Nikolaevna Golubeva, Elena

Nikolaidou, Thalia

Nikolakopoulos, Konstantinos

Nikolic, Dejan

Nikolic, Jovanka

Nikolopoulos, Dimitrios

Nikolov, Ned

Nikonorov, Artem

Nilsson, Johan

Nilsson, Tobias 
Nina, Aleksandra

Ningthoujam, Ramesh

Nino, Pasquale

Ninyerola, Miquel

Nir, Sapir

Niraula, Rewati

Niro, Fabrizio

Niroumand-Jadidi, Milad

Nisa, Zaibun

Nishimura, Toshi

Nishitsuji, Yohei

Nistor, Sorin

Nita, Mihai

Niu, Haoyu

Niu, Yanbo

Niu, Zhenguo

Noack, Markus

Noack, Patrick Ole

Nobile, Adriano

Noce, Sergio

Nocelo Lopez, Ruben

Nocentini, Alessandro

Nocerino, Erica

Nodzu, Masato

Noe, Steffen M.

Noh, Seong Jin

Noh, Yoo-Jeong

Noh, Young-Chan

Noh, Young-Min

Nolet, Corjan

Nölke, Nils

Nones, Michael

Nooni, Isaac

Nordman, Maaria

Normandin, Cassandra

Noroozinejad Farsangi, Ehsan

Norton, Alexander J.

Norton, Michael

Nosenko, Gennady A.

Noszczyk, Tomasz

Notarpietro, Riccardo

Notti, Davide

Nouri, Amin

Novak, Pavel

Novelli, Antonio

Novellino, Alessandro

Novo, Ana

Novo, Evlyn

Novo, Evlyn Moraes

Nowak, Maciej

Nowak, Marcin
Nowakowski, Artur

Nowosielski, Leszek

Nozawa, Satonori

Nozzoli, Francesco

Ntouskos, Valsamis

Numbisi, Frederick N.

Nunes, Leonel Jorge Ribeiro

Nunes, Luis

Nuñez-Andrés, Amparo

Nunziata, Ferdinando

Nüsser, Marcus

Nuyts, Siegmund

Nyadjro, Ebenezer

Nyka, Krzysztof

Nykiel, Grzegorz

Nyland, Kelsey

O'Banion, Matt

O'Byrne, Michael

O'Connell, Jessica

Oats, Renee

Obanawa, Hiroyuki

Obata, Kenta

Obland, Michael

Obolewski, Krystian

Obradovic, Ratko

Obregón, Maria A.

Obringer, Renee

Ochoa, Andrés

Odetti, Angelo

Odijk, Dennis

Odindi, John

Odry, Ákos

Odziemczyk, Waldemar

Ogashawara, Igor

Ogawa, Daisuke

Ogorodov, Stanislav

Ogryzek, Marek

Oguchi, Takashi

Ogut, Mehmet

Ogutu, Booker

Oh, ByungTae

Oh, Seoung-Jun

Oh, Sungchan

Oh, Sungmin

Oh, Taekeun

Ohara, Noriaki

Ohigashi, Tadayasu

Ohtonen, Olli

Ohya, Hiroyo

Ohyama, Hirofumi

Oishi, Yu 
Ojaroudi Parchin, Naser

Ojha, Chandrakanta

Ojo, Adegbola

Okabe, Takumi

Okada, Kazuya

Okarma, Krzysztof

Okeke, Godwin Amechi

Okhrimenko, Maxim

Oksanen, Elina

Oktem, Rusen

Okuyama, Arata

Olah, Branislav

Olaizola, Igor García

Olander, Timothy L.

Olchev, Alexander

Oleynik, Philipp

Oliphant, Adam

Oliva, Diego

Olivares, Barlin Orlando

Oliveira Assis, Talita

Oliveira, Anabela

Oliveira, Dário Augusto Borges

Oliveira, João F.

Oliveira, Julianne

Oliveira, Rui Jorge

Oliveira-Júnior, José Francisco

Oliveras, Paz Fernández

Olli, Kalle

Ololade, Olusola

Olsson, Håkan

Olugbara, Oludayo

Olyazadeh, Roya

Omani, Nina

Omara, Ibrahim

Ombadi, Mohammed

Omidalizarandi, Mohammad

Omrani, Hichem

Onaca, Alexandru

Oniga, Florin

Oniga, Valeria-Ersilia

Ono, Masahiro

Ontel, Irina

Onyutha, Charles

Oppelt, Natascha

Opromolla, Roberto

Orchard, Shane

Ordoñez, Celestino

Orellana-Alvear, Johanna

Orhei, Ciprian

Orikasa, Narihiro

Orlando, Danilo
Ornella, Leonardo

Orosei, Roberto

Orović, Irena

Orozco, Ulises

Ortega-Fernández, Francisco

Ortega-Huerta, Miguel

Ortenzi, Luciano

Ortiz, Francisco

Ortiz, Luis

Ortiz-Coder, Pedro

Ortolani, Alberto

Ortolani, Michele

Ortolani, Sergio

Ortolano, Gaetano

Osadebey, Michael

Osco, Lucas Prado

Oshtorjani, Ehsan Kiani

Osińska-Skotak, Katarzyna

Ospina, Raydonal

Osródka, Leszek

Oštir, Krištof

Ostovari, Yaser

Ostrowski, Rafał

Oszako, Tomasz

Oszust, Mariusz

Otepka, Johannes

Othman, Arsalan

Otis, Daniel

Otremba, Zbigniew

Otsuka, Yuichi

Ott, Peter

Ottaviano, Marco

$\mathrm{Ou}, \mathrm{Chia}-\mathrm{Ho}$

Ouaadi, Nadia

Ouahabi, Abdeldjalil

Ouma, Yashon O.

Ou-Yang, Chang-Feng

Ouyang, Chaojun

Ouyang, Yen-Chieh

Ovando, Gustavo

Oveis, Amir Hosein

Oviedo De La Fuente, Manuel

Øvstedal, Ola

Owens, Phillip

Ozanich, Emma

Özdemir, Emre

Özendi, Mustafa

Ozgokman, Tamay

Paar, Gerhard

Paar, Rinaldo

Pace, Giandomenico 
Pachepsky, Yakov

Pachidis, Theodore

Paço, Teresa A.

Pacuta, Vladimiradilla, Oswaldo

Paczos-Grzeda, Edyta

Padilla-Vivanco, Alfonso

Padman, Laurence

Padmanaban, Rajchandar

Padokhin, Artem

Padovan, Sebastiano

Padró, Joan-Cristian

Padua, Luis

Paduan, Jeff

Paek, Sung Wook

Páez, Raúl

Pagán, Brianna R.

Paganelli, Filippo

Pagay, Vinay

Paget, Matt J.

Paggi, Horacio

Pagiatakis, Spiros

Pail, Roland

Painho, Marco

Pajak, Katarzyna

Pajdak-Stós, Agnieszka

Pajić, Vladimir

Pal, Subodh Chandra

Palacios, Guillermo

Palacios-Peña, Laura

Palacios-Rodriguez, Guillermo

Palaiologou, Palaiologos

Palamuleni, Lobina

Palaseanu-Lovejoy, Monica

Palerme, Cyril

Palermo, Michele

Palharini, Rayana

Paliaga, Guido

Paliwal, Ambica

Pallikarakis, Aggelos

Pallotta, Luca

Palme, Massimo

Palmer, Diane

Palmerini, Giovanni

Palombo, Angelo

Paloscia, Simonetta

Pamart, Anthony

Pamucar, Dragan

Pamuła, Teresa

Pan, Hong

Pan, Jeng-Shyang

Pan, Jiayi
Pan, Jinmei

Pan, Laura L.

Pan, Maohua

Pan, Tao

Pan, Xianzhang

Pan, Xiaoduo

Pan, Xiaohua

Pan, Yiwen

Pan, Zhigang

Panagiotakis, Costas

Panagiotidis, Dimitrios

Panagopoulos, Thomas

Panagos, Panos

Panahi, Mahdi

Panda, Sudhanshu Sekhar

Pandey, Manish

Pandey, Prem Chandra

Pandzic, Kreso

Paneque, Manuel

Pang-jo, Chun

Pani, Shantanu Kumar

Panić, Branislav

Panicker, Sabita

Panidi, E. A.

Panneton, Bernard

Pannone, Daniele

Pantaloni, Marco

Pantazi, Xanthoula Eirini

Pantazis, Dimos N.

Paola, Carrara

Paolini, Christopher

Paolo, Russo

Papacharalampous, Georgia

Papadakis, Vassilis

Papadopoulos, Gerassimos A.

Papadopoulos, Nikos

Papageorgiou, Dimitris

Papagiannopoulos, Nikolaos

Papakonstantinou, Apostolos

Papalexiou, Simon Michael

Papandrea, Enzo

Papanikolaou, Thomas

Papathanasiou, George

Papayannis, Alexandros

Papenmeier, Svenja

Papić, Vladan

Pappas, Odysseas A.

Pappu, Chandra

Parajka, Juraj

Paramonov, Alexander

Parcharidis, Issaak 
Pardini, Matteo

Pardo, Mario

Paredes-Trejo, Franklin

Parente, Leandro Leal

Parida, Bikash Rajan

Paris, Claudia

Parisi, Erica Isabella

Parizzi, Alessandro

Park, Chae Yeon

Park, Edward

Park, Haemi

Park, Hoonyoung

Park, Hyuk

Park, Jaehwa

Park, Jae-Hyoung

Park, Jeonghong

Park, Jeonghwan

Park, Jeong-Soo

Park, Ji-Eun

Park, Jihye

Park, Jinku

Park, Jisoo

Park, Jong-Hwa

Park, Jongmin

Park, Kyunghun

Park, Minsu

Park, Moojong

Park, Moon-Soo

Park, Seonho

Park, Sumin

Park, Suyoung

Park, Yongeun

Parker, Jackie

Parlagreco, Luca

Parlow, Eberhard

Parmehr, Ebadat Ghanbari

Parmentier, Alexandra

Parmes, Eija

Parra, Lorena

Parra, Rene

Parraga, Adriane

Parrens, Marie

Parrini, Francesca

Parrot, Michel

Paršeliünas, Eimuntas Kazimieras

Parshin, Alexander

Parsons, Russell

Parsova, Velta

Parvizi, Roohollah

Pârvulescu, Lucian

Pascual, Daniel
Pascual, Josep

Pascucci, Simone

Pashaei, Mohammad

Pashova, Lyubka

Pasken, Robert

Pasolini, Gianni

Pasolli, Edoardo

Pasquali, Davide

Pasquali, Paolo

Passerini, Giorgio

Pastena, Massimiliano

Pasternak, Galia

Pastor Guzman, Francisco

Pastor-Escuredo, David

Pastor-Guzman, Julio

Pastorino, Martina

Pastor-López, Iker

Paszkiewicz, Andrzej

Pasztaleniec, Agnieszka

Pásztor, László

Patel, Nirav Nikunj

Patella, Domenico

Pathak, Rohit

Patias, Petros

Patil, Ashok Kumar

Patil, Ujwalkumar

Patino, Jorge

Patočka, Zdeněk

Patrone, Fabio

Patrucco, Giacomo

Patruno, Jolanda

Pau, Giovanni

Paudel, Basanta

Paugam, Ronan

Paul, George

Paul, Seema

Paulette, Laura

Pauly, Klaas

Pauselli, Cristina

Pavan Kumar, B. N.

Pavasović, Marko

Pavel, Svec

Pavelka, Karel

Pavese, Franco

Pavlić, Krešimir

Pavlides, Andrew

Pavlidou, Efthymia

Pavlis, Nikolaos K.

Pavliuk, Roman

Pavloudakis, Francis

Pavlovčič-Prešeren, Polona 
Pawlik, Łukasz

Pawluszek, Kamila

Payá, Luis

Pay-Liam, Lin

Paynter, Ian

Payo, Andres

Paz Pellat, Fernando

Paz, Igor

Paziewski, Jacek

Peano, Daniele

Pearlman, Aaron

Pebesma, Edzer

Pechanec, Vilem

Peckhaus, Andreas

Pecoraro, Gaetano

Pedersen, Leif Toudal

Pedreño, Jose Navarro

Peduzzi, Alicia

Peet, Jacob C. A. Van

Pegau, Scott

Peik, Bijan

Peirano, Andrea

Peláez, José A.

Pelc-Mieczkowska, Renata

Pellegrinelli, Alberto

Pelletier, Charlotte

Pelletier, Mathew G.

Pellitero, Ramón

Pelta, Ran

Pelto, Mauri

Peltoniemi, Jouni

Peña, Carlos

Peña-Cruz, Manuel I.

Pendock, Neil

Peng, Chao-Chung

Peng, Chuang

Peng, Daifeng

Peng, Dailiang

Peng, Dingzhi

Peng, Jie

Peng, Jingjing

Peng, Pingan

Peng, Xi

Peng, YuXiang

Peng, Zhengyu

Peng, Zhenming

Penna, Nadia

Pennino, Silvia

Penttilä, Antti

Pepe, Antonio

Pepe, Massimiliano
Pepe, Monica

Peppas, Kostas

Pere, Jean-Christophe

Pereira, Luisa Gomes

Pereira-Sandoval, Marcela

Perera, Asanka G.

Perera, Kithsiri

Perevalova, Natalia

Perez Cruzado, Cesar

Pérez De Prado, Rocío Josefina

Pérez, Emiliano

Pérez, Isidro A.

Pérez-Brunius, Paula

Pérez-Bueno, Fernando

Pérez-Cabello, Fernando

Perez-Gracia, Vega

Pérez-Martín, Enrique

Pérez-Morales, Alfredo

Pérez-Peña, José Vicente

Perez-Perez, Yeritza

Pérez-Planells, Lluís

Pérez-Porras, Fernando

Pérez-Rodríguez, Fernando

Pérez-Sánchez, Julio

Perez-Verdin, Gustavo

Pergola, Nicola

Perilli, Angelo

Perko, Roland

Perković, Dario

Perkovic, Marko

Perlwitz, Jan P.

Perna, Massimo

Pernão, João

Pernek, Milan

Péron, Guillaume

Peron, Roberto

Perotto, Humberto L.

Perrone, Daniele

Perronne, Rémi

Perroy, Ryan L.

Perry, Stuart

Persad, Ravi Ancil

Persico, Raffaele

Persson, Henrik J.

Pertsev, Nikolay

Pescaru, Dan

Pesci, Arianna

Peshev, Zahary

Petcu, Dana

Peter, Brad G.

Peter, Heike 
Peters, Dennis K.

Petersen, Gudrún Nína

Peterson, Birgit

Peterson, Seth H.

Petit, Michel

Pętlicki, Michał

Petrariu, Adrian Ioan

Petras, Vaclav

Petrellis, Nikos

Petrila, Marius

Petrini, Paolo

Petris, Samuele De

Petrisor, Alexandru-Ionut

Petropoulos, George P.

Petropoulou, Christy Chryssanthi

Petrova, Irina Yu.

Petrovič, František

Petsa, Elli

Pettersen, Claire

Pettinato, Simone

Peyre, Gwendolyn

Peyret, M.

Pezzi, Luciano Ponzi

Pezzo, Giuseppe

Pezzopane, Michael

Pezzutto, Simon

Pfeil, Isabella

Pfitzner, Kirrilly

Pflug, Bringfried

Phalke, Aparna

Pham, Lien Thi Hong

Pham, Quoc Bao

Pham, Tien Dat

Phelan, Brian

Phyo, Cho Nilar

Piao, Daquing

Picchi, Gianni

Picchio, Francesca

Picchio, Rodolfo

Piccini, Chiara

Picco, Lorenzo

Pichon, Lionel

Pickering, Kenneth E.

Picone, Marco

Picos, Juan

Picot, Nicolas

Picotte, Josh

Picotte, Joshua J.

Piechota, Thomas C.

Piekutowska, Magdalena

Pielke, Roger, Sr.
Piemonte, Andrea

Pierdicca, Roberto

Piermattei, Livia

Piersanti, Mirko

Pieruccioni, Diego

Pietrella, M.

Pietron, Marcin

Pietropaolo, Ermanno

Pietrosemoli, Ermanno

Pignalberi, Alessio

Pignatti, Stefano

Pikuła, Dorota

Pilant, Andrew

Pilarska, Magdalena

Pilaš, Ivan

Pilato, Antonio Di

Pilecka, Elżbieta

Pimentel, Rafael

Pimm, Stuart L.

Pina, Pedro

Pinat, Elisa

Pinchas, Monika

Pineda, Mónica

Pinedo-Alvarez, Alfredo

Pinhasi, Yosef

Pinho, Marcelo

Pinkovykiy, Maxim

Pinnel, Nicole

Pintilii, Radu-Daniel

Pinto, Joseph

Pinto, Milena Faria

Pinto, Victor A.

Pintore, Giovanni

Pinto-Roa, Diego P.

Pio, Gianvito

Piotr, Lichota

Pirazzini, Roberta

Pirchio, Michel

Pires De Lima, Rafael

Pires, Ivan Miguel

Piroddi, Luca

Pirotti, Francesco

Pirrone, Davide

Pisante, Michele

Pishchalnikov, Roman Y.

Piskozub, Jacek

Pisoni, Enrico

Pisoni, Juan Pablo

Pitalúa-Díaz, Nun

Pitarch, Jaime

Pitonak, Martin 
Pla, Magda

Płaczkowska, Eliza

Plado, Jüri

Plant, Genevieve

Plaščak, Ivan

Platov, Gennady

Plavšić, Jasna

Pleniou, Magdalini

Plescan, Costel

Plescia, Jeffrey

Pleskachevsky, Andrey

Pluta, Kacper

Plutino, Manuela

Pnevmatikos, Nikos

Poblete-Echeverria, Carlos

Podd, Frank J. W.

Poděbradská, Markéta

Podstrigaev, Alexey

Poenaru, Violeta

Poggi, Giovanni

Poggiali, Valerio

Pohl, Walter Leopold

Pokorný, Jiří

Pokswinski, Scott

Połap, Dawid

Polcari, Marco

Polehampton, Edward

Poley, Lucy G.

Polidori, Laurent

Polishchuk, Yury

Polkowska, Zaneta

Polla, Silvia

Pollino, Maurizio

Połom, Marcin

Pölönen, Ilkka

Polverari, Federica

Polyakov, Alexander

Polykretis, Christos

Pompa-García, Marín

Ponce De León, Sonia

Ponce, Octavio

Ponce-Hernandez, Raul

Poncet, Aurelie

Ponchak, George

Ponciano, Jean-Jacques

Poniszewska-Maranda, Aneta

Ponomarev, Eugene

Ponomaryov, Volodymyr

Ponte, Salvatore

Pontes, Montcharles Da Silva

Ponti, Cristina
Poovvancheri, Jiju

Popescu, Dan

Popielarczyk, Dariusz

Popov, Sergey V.

Popović, Luka Č.

Poppiel, Raúl Roberto

Popstefanija, Ivan

Porcelli, Luca

Porcù, Federico

Poreh, Davod

Portapila, Margarita

Portero, Jaime Pitarch

Pospichal, Bernhard

Postacchini, Matteo

Potortì, Francesco

Pottie, Paul-Eric

Potůčková, Markéta

Poulose, Alwin

Poupardin, Adrien

Pour, Amin Beiranvand

Pourghasemia, Hamid Reza

Pourreza, Alireza

Poursanidis, Dimitris

Povey, Adam

Povilanskas, Ramūnas

Powell, Rebecca

Powis, Terry

Pradhan, Biswajeet

Pradhan, Nawa Raj

Pradhan, Yaswant

Pradhanang, Soni

Prakash, Nikhil

Prakash, Satya

Pranzini, Enzo

Prasad, Abhnil

Prasad, Mukesh

Prates, Gonçalo

Prati, Ronaldo

Praticò, Salvatore

Prazuch, Janusz

Pregowska, Agnieszka

Premachandra, Chinthaka

Prestileo, Fernanda

Prestininzi, Pietro

Preval, Simon P.

Previtali, Mattia

Price, Owen

Prieto, Andrés J.

Prieto, Juan F.

Prieto-Amparán, Jesús Alejandro

Pringle, James M. 
Prinz, Rainer

Putman, Philip

Putzu, Lorenzo

Puziene, Ruta

Pyka, Krystian

Pyo, Jongcheol

Pyrchla, Jerzy

Qadri, Syed Muhammad Talha

Qadri, Talha

Qamar, Shamimul

Qayyum, Abdul

Qi, Guanqiu

Qi, Hongshuai

Qi, Jianbo

Qi, Wenlu

Qi, Youcun

Qi, Zhixin

Qian, Guoqi

Qian, Yulei

Qiao, Jing

Qin, Anzhen

Qin, Hui

Qin, Kai

Qin, Wenmin

Qin, Yi

Qin, Yuchu

Qiu, Guoyu

Qiu, Sen

Qiu, Tong

Qiu, Xiaolan

Qiu, Yuanying

Qiu, Yubao

Qu, Feifei

Qu, Zhipeng

Quan, Xingwen

Quartulli, Marco

Quast, Raphael

Queißer, Manuel

Quej, Victor H.

Quesada-Barriuso, Pablo

Quesada-Román, Adolfo

Quevedo-Gutiérrez, Eduardo

Quinn, Nigel William Trevelyan

Quinn, Rory

Quintanar, Arturo

Quinta-Nova, Luís

R. Buchholz, Rebecca

Rabah, Hassan

Rabbel, Wolfgang

Rabbi, Jakaria

Rabby, Yasin Wahid
Raber, George

Rabiee, Ramtin

Rabin, Bob

Racetin, Ivana

Racoviteanu, Adina

Rączkowski, Włodzimierz

Radhakrishnan, Chandrasekar

Radiarta, I Nyoman

Radicioni, Fabio

Radkevich, Alexander V.

Radkowski, Adam

Radočaj, Dorijan

Radoglou-Grammatikis, Panagiotis

Radoi, Anamaria

Radosavljevic, Zvonko

Radoux, Julien

Radovanovic, Milan

Radulescu, Virgil Mihai

Radulović, Aleksandra

Radwan, Taher M.

Rafa, Mickey

Raffa, Francesco

Rafieeinasab, Arezoo

Ragettli, Silvan

Rahaghi, Abolfazl Irani

Rahaman, S Abdul

Rahman, Atiqur

Rahman, Habib

Rahman, Mizanur

Rahman, Moshiur

Rahman, Muhammad

Rahman, Muhib

Rahman, Munshi Khaledur

Rahmati, Mehdi

Raida, Zbynek

Raimondi, Valentina

Räisänen, Petri

Raissouni, Naoufal

Raiyani, Kashyap

Raj Bista, Suman

Raj Meena, Sansar

Raj, Nawin

Raj, Rahul

Rajapakshe, Chamara

Rajasekaran, Eswar

Rakić, Dragan

Rakitin, Vadim

Rakoczy, Anna M.

Rallo, Giovanni

Ramadan, Rabie A.

Ramadhani, Fadhlullah 
Ramage, Joan

Raman, Aishwarya

Ramanauskaite, Simona

Ramapriyan, Hampapuram

Ramella, Giuliana

Ramezan, Christopher

Ramillien, Guillaume

Ramirez, Juan Marcos

Ramirez, Ryan

Ramírez-Cuesta, Juan Miguel

Ramírez-Rojas, Alejandro

Ramoelo, Abel

Ramos, Maria Isabel

Ramos, Victor

Rampa, Vittorio

Ran, Youhua

Rana, Fabio Michele

Rana, Gianfranco

Rana, Parvez

Ranagalage, Manjula

Randazzo, Giovanni

Ranieri, Gaetano

Ranndal, Heidi

Rao, Ajith

Rao, Douglas

Raouf, Abdul

Rapant, Petr

Rapinchuk, Ekaterina

Rapiński, Jacek

Rapoport, Yuriy G.

Raposo, Mauro

Raptis, Dimitrios

Raptis, Ioannis-Panagiotis

Rasch, Joel

Rasche, Christoph

Rascon, Caleb

Rashidi, Maria

Raši, Rastislav

Rasol, Mezgeen

Rašovský, Marek

Raspollini, Piera

Rasquinha, Dina

Rasti, Behnood

Raszka, Beata

Rato, Luis

Ratsimandresy, Andry

Raudonis, Vidas

Raudsepp, Urmas

Rauf, Hafiz Tayyab

Ravankar, Ankit

Ray, Jagat Dwipendra
Raybaut, Myriam

Raykova, Reneta

Raynolds, Martha

Raynor, Edward

Razevig, Vladimir

Reagan, James

Realmuto, Vincent

Realpe, Miguel

Reboul, Serge

Reddy, Venugopal Reddy Thandlam

Redweik, Paula

Reed, Denise

Rees, Alan F.

Rees, Gareth

Refaat, Tamer F.

Refice, Alberto

Regnard, Jean-Luc

Reiche, Johannes

Reineix, Alain

Reinhardt, Jason

Reinisch, Elena C.

Reinoso-Rondinel, Ricardo

Reis, Manuel J. C. S.

Reis, Mariane S.

Reiterer, Alexander

Rejfek, Lubos

Remy, Dominique

Rémy, Samuel

Ren, Christopher Xiang

Ren, Chunying

Ren, Hai Peng

Ren, Lei

Ren, Peng

Ren, Shisong

Ren, Zhengyong

Renard, Jean-Baptiste

Rende, Sante Francesco

Rendenieks, Zigmārs

Renné, David S.

Rennie, Susan

Renza, Diego

Resano, Javier

Reshitnyk, Luba

Resmini, Ronald

Resop, Jonathan

Restaino, Rocco

Restas, Agoston

Retalis, Adrianos

Retterer, John Michael

Reul, Nicolas

Reuma, Arav 
Reusch, Stefan

Reuter, Hannes

Revill, Andrew

Rex, Franciel Eduardo

Reyes-Aldasoro, Constantino Carlos

Reyes-Carmona, Cristina

Reyes-González, Arturo

Reynier, Philippe

Rezaei, Ehsan Eyshi

Rezende, Luiz Felipe C.

Řzník, Tomáš

Rhea, Lee K.

Rhinane, Hicham

Rhodes, Edward

Rial, Fernando

Riaza, Asuncion

Riba, Jordi-Roger

Ribal, Agustinus

Ribas, Salvador

Ribeiro Neto, Alfredo

Ribeiro Pinto, João

Ribeiro, Eduardo Augusto Werneck

Ribeiro, Matheus Henrique Dal Molin

Ribeiro, Sergio Silva

Ribeiro, Tiago

Ribichini, Remo

Ribodetti, Alessandra

Ribolini, Adriano

Ricchi, Antonio

Ricciardelli, Elisabetta

Richards-Rissetto, Heather

Richetti, Jonathan

Richter, Andreas

Richter, Rudolf

Rida, Imad

Riddick, Caitlin

Rieg, Lorenzo

Riel, Bryan

Rifat, Shaikh Abdullah Al

Rigge, Matthew

Riggs, George

Righi, Marco

Righi, Rodrigo

Rigo, Tomeu

Riihelä, Aku

Rijal, Santosh

Riksen, Michel

Rimal, Bhagawat

Rimando, Jeremy

Ringerud, Sarah

Rios, Carlos
Risbøl, Ole

Risch, Eric

Risojevic, Vladimir

Risser, Mark

Rissolo, Dominique

Ristic-Durrant, Danijela

Ritter, Christoph

Ritter, Tim

Rivalland, Vincent

Rivas, Marga L

Rivas, Raúl

Rivas-Tabares, David

Rivera, Francisco Fernández

Rizaldy, Aldino

Rizza, Umberto

Rizzo, Rodnei

Robert, Tenzer

Roberti, Debora Regina

Roberts, Allen D.

Roberts, Dar

Roberts, Roger

Robertson, Kevin M.

Robinson, Nathan

Robles, Ramiro

Rocca, Fabio

Roccati, Anna

Rocha, Alby Duarte

Rocha, Alfredo

Rocha, Cesar

Rocha, João

Rocha, Jorge

Roche, Jamie

Rocher, Javier

Rochon, Yves J.

Roda, Jean-Marc

Rodella, Ilaria

Rodning, Chris

Rodrigo Comino, Jesús

Rodrigo, Juan Fernando

Rodrigues, Armanda

Rodrigues, Carla Pimental

Rodrigues, Clara F.

Rodrigues, Thanan

Rodríguez Sousa, Antonio Alberto

Rodríguez Temiño, Ignacio

Rodriguez, Andrea

Rodríguez, Demóstenes Zegarra

Rodriguez, Dionisio

Rodríguez, José-Víctor

Rodríguez, Orlando Camargo

Rodriguez, Roberto 
Rodriguez-alvarez, Nereida

Rodríguez-Avi, José

Rodríguez-Baena, Domingo Savio

Rodriguez-Fernandez, Nemesio

Rodríguez-Gonzálvez, Pablo

Rodríguez-Lizana, Antonio

Rodriguez-Martin, Manuel

Rodriguez-Moreno, Victor M.

Rodriguez-Perez, Daniel

Rodriguez-Rastrero, Manuel

Rodríguez-Velasco, Gema

Roeder, William P.

Roelfsema, Chris

Roessler, Sebastial

Rogeau, Marie-Pierre

Roggero, Marco

Rogowski, Peter

Roh, Kyoung-Min

Roić, Miodrag

Roig, Ignacio Bosch

Roisenberg, Mauro

Rojas, Octavio

Rojo Baio, Fábio Henrique

Rokicki, Tomasz

Rolim, João

Rollenbeck, Rütger

Roma-Dollase, David

Romain, Olivier

Roman, Kamil

Roman-Gonzalez, Avid

Romano, Filomena

Romano, Giovanni

Romano, Salvatore

Romanov, Andrey

Romanov, Peter

Romanovskii, Oleg A.

Roman-Stork, Heather

Romero, Julio

Romero, Silvia

Romero-Sanchez, Martin

Romero-Sanchez, Martin Enrique

Romić, Marija

Roncella, Riccardo

Ronchetti, Giulia

Ronga, Domenico

Rönnholm, Petri

Ronsivalle, Daniele

Root, Bart

Ropelewska, Ewa

Roque, Dora

Rosa, Katia
Rosário, Denis

Rosca, Sanda

Rose, Paul

Roselli, Ivan

Rosenfeld, Daniel

Rosi, Ascanio

Rosiński, Adam

Ross, Jeremy

Rossato, Luciana

Rossetti, Silvia

Rossi, Lorenzo

Rossini, Luca

Rößler, Sebastian

Rostami, Mohammad

Roth, Guy

Rotman, Stanley

Rotta, Luiz

Rotunno Filho, Otto Corrêa

Roueff, Antoine

Roujean, Jean-Louis

Roumelioti, Zafeiria

Roundy, Paul

Roupioz, Laure

Rousseau, David

Roussos, Evangelos

Rousta, Iman

Rowe, Elizabeth

Rowley, Nathan

Roy, Parth Sarathi

Rozario, Papia

Rozos, Evangelos

Rozsa, Zoltan

Różycki, Sebastian

Ruban, Dmitry

Rubanenko, Lior

Ruberti, Daniela

Rubio, Cristina

Rubio, Jose De Jesus

Rucci, Alessio

Ruddick, Kevin

Rudenko, Sergei

Rudinskas, Darius

Rueda-Roa, Digna T.

Ruefenacht, Bonnie

Ruescas, Ana Belen

Ruffell, Alastair

Rufin, Philippe

Rufino, Giancarlo

Ruhtz, Thomas

Ruiz Armenteros, Antonio Miguel

Ruiz, Oscar 
Ruiz-De-Azúa, Joan Adrià

Ruiz-Etcheverry, Laura A.

Ruiz-Munoz, Jose Francisco

Ruiz-Sinoga, José Damián

Rumora, Luka

Runyon, Kirby

Ruokolainen, Kalle

Rusca, Florin Valentin

Rusnák, Miloš

Russell, Brandon

Sabbagh, Dario

Saber, Mohamed

Saberioon, Mohammadmehdi

Sabharwal, Chaman

Sabol, Donald

Sachs, Jürgen

Sadeghi-Niaraki, Abolghasem

Sadjadi, Firooz

Sadowski, Maciej

Saeed, Khalid

Safanelli, José Lucas

Safonov, Ilia

Safronov, Alexander N.

Sagade, Atul

Sagan, Vasit

Saha, Korak

Saha, Provat

Saha, Sangeet

Saha, Sudipan

Sahajpal, Ritvik

Saher, Rubab

Sahin, Caner

Saho, Kenshi

Sahoo, Alok

Sahoo, Soumya R.

Sahour, Hossein

Sahu, Netrananda

Sahwan, Wahib

Saifuzzaman, Md

Saintenoy, Albane

Saito, Masanori

Sajjadian, Masoud

Sakai, Takeyasu

Sakai, Toru

Sakamoto, Maiko

Sakatani, Naoya

Sakib, Mohiuddin

Sakib, Salman

Sakic, Pierre

Sakpere, Wilson

Sakuno, Yuji
Sala, Florin

Salamanis, Athanasios

Salamí, Esther

Salas, Eric

Salata, Stefano

Salata, Tomasz

Salati, Sanaz

Salauddin, Md

Salazar, Addisson

Salazar, Pablo

Saleem, Kashif

Saleem, Nayyer

Salekin, Serajis

Salem, Ali

Salem, Milad

Salem, Salem Ibrahim

Saleous, Nazmi

Salerno, Emanuele

Salgueiro Romero, Luis

Salio, Paola

Salvati, Luca

Salvatori, Rosamaria

Salvini, Riccardo

Salzano, Roberto

Samaniego, Franklin

Samanta, Dhrubajyoti

Samanta, Sailesh

Samardžić-Petrović, Mileva

Samat, Alim

Sambito, Mariacrocetta

Samczynski, Piotr

Samiappan, Sathishkumar

Samiei, Salma

Samimi-Namin, Kaveh

Sampath, Dissanayake

Sampietro, Daniele

Sams, Brent

Samsonov, Timofey E.

Samuel, John

San Bautista, Alberto

San Jose, Roberto

San Martin, Narkis Morales

San, Bekir Taner

Sánchez Bellón, Ángel

Sanchez De La Flor, Francisco José

Sánchez De Miguel, Alejandro

Sanchez Galan, Javier

Sanchez, Alber

Sanchez, Alejandro

Sanchez, Juanma Lopez

Sanchez, Nilda 
Sanchez, Noelia

Sanchez, Thomas

Sanchez, Victor

Sánchez, Yolanda

Sanchez-Aguero, Victor

Sánchez-Alzola, Alberto

Sánchez-Aparicio, Luis Javier

Sánchez-Carnero, Noela

Sanchez-Cuevas, Pedro J.

Sánchez-Fernández, Manuel

Sánchez-Gómez, Mario

Sánchez-Rodríguez, Ana

Sánchez-Ruiz, Sergio

Sánchez-Sastre, Luis Fernando

Sandberg, Marcus

Sander De Carvalho, Lino Augusto

Sandhu, Karansher

Sandino, Juan

Sandlersky, Robert

Sandoval-Herazo, Luis Carlos

Şandric, Ionuț

Sangirardi, Marialuigia

Sanjiwana, Arjasakusuma

Sanjou, M.

Sanli, Fusun

Sanna, Francesca

Sanquetta, Carlos

San-Segundo, Rubén

Santamaría, Luis

Santamaría-Peña, Jacinto

Santana Maia, Deise

Santana, Oliverio J.

Santangelo, Nicoletta

Santarsiero, Valentina

Santi, Emanuele

Santi, Fabrizio

Santini, Federico

Santoro, Mattia

Santoro, Simone

Santos Assunção, Sónia Alexandra

Santos, Angela

Santos, Daniel Rodrigues Dos

Santos, Florentino

Santos, José Gomes Dos

Santos, Marcelo C.

Santos, Max Mauro Dias

Santos, Rafael

Santos, Rodrigo M.

Santra, Avik

Säntti, Tero

Sañudo, Roberto
Saotome, Osamu

Sapana, Lohani

Sapena, Marta

Sapkota, Bishwa

Saponaro, Mirko

Sapountzis, Marios

Sapucci, Luiz Fernando

Sara, Pensieri

Saragadam, Vishwanath

Saraswati, Anita Thea

Saravanan, T. Jothi

Sarigiannidis, Panagiotis

Sarker, Chandrama

Šarlah, Nikolaj

Sarlis, Nicholas

Saroli, Michele

Sarría, Francisco Alonso

Sarris, Apostolos

Sarvia, Filippo

Sasa, Kenji

Sasaki, Nophea

Sasano, Masahiko

Sasi, Sruthy

Sathyachandran, Sanath Kumar

Sato, Kazutoshi

Sato, Ryoichi

Satriano, Valeria

Sattar, Farook

Saudreau, Marc

Saunders, Roger

Sautot, Lucile

Savard, Genevieve

Savchuk, Stepan

Saveliev, Anatoly

Savenets, Mykhailo

Savi, Patrizia

Savin, Igor

Savin, Igor $\mathrm{Yu}$.

Savtchenko, Andrey K.

Sawada, Yohei

Sawyer, Virginia Ruth

Saxe, Samuel

Sayab, Mohammad

Saylam, Kutalmis

Sayyouri, Mhamed

Sazib, Nazmus

Scafetta, Nicola

Scala, Barbara

Scalenghe, Riccardo

Scalici, Massimiliano

Scaradozzi, David 
Scarascia-Mugnozza, Giuseppe

Scardino, Giovanni

Scaringi, Gianvito

Scarnati, Theresa

Scarpa, Gian Marco

Scarpa, Giuseppe

Scarpa, Roberto

Scavuzzo, Carlos M.

Schäfer, Klaus

Schaff, Florian

Scharff, Lea

Scharffenberg, Martin

Scharringhausen, Marco

Schedl, David C.

Schepaschenko, Dmitry

Schiappacasse, Paulina

Schiefler, Nivaldo T.

Schillaci, Calogero

Schillak, Stanisław

Schimalski, Marcos

Schimalski, Marcos Benedito

Schindler, Mirjam

Schirrmann, Michael

Schirru, Luca

Schlaepfer, Daniel

Schlicht, Anja

Schlögl, Matthias

Schlund, Michael

Schlüter, Stephan

Schmalzel, John

Schmid, Moritz S.

Schmidt-Thome, Philip

Schmit, Timothy J.

Schmitt, Thierry

Schmitz, Berit

Schneider, Mathias

Schofield, Gail

Scholten, Olaf

Schramm, Michael

Schreiber, K. Ulrich

Schreier, Mathias

Schreiner-McGraw, Adam

Schröder, Dietrich

Schröder, Susanne

Schroder, Whittaker

Schroeder, Dustin

Schubert, Matthias

Schuddeboom, Alex

Schuh, Harald

Schulthess, Urs

Schultz, Johannes
Schumacher, Johannes

Schumann, Guy J.-P.

Schurwanz, Max

Schvartzman, David

Schwaab, Jonas

Schwarz, Gottfried

Schwarz, Jill

Schwarz, Roland

Sciarra, Nicola

Scifoni, Silvia

Scott, Andrea

Scott, Deron

Scotto, Carlo

Scuderi, Louis

Scully, Brandan

Seal, Ayan

Sears, Michael

Sebari, Imane

Sebastian, Jucu Ioan

Šebeň, Vladimír

Sebestyen, Gheorghe

See, Linda

Seela, Balaji

Segarra, Joel

Segers, Laurent

Segoni, Samuele

Seguini, Lorenzo

Seidel, Dominik

Seidel, Felix

Seidel, Michael

Seingier, Georges

Seip, Knut L.

Seitsonen, Oula

Sekertekin, Aliihsan

Sekrecka, Aleksandra

Seleiman, Mahmoud F.

Sellami, Akrem

Selvaraj, Michael Gomez

Semenishchev, E. A.

Semeniuta, Oleksandr

Semeraro, Teodoro

Semiromi, Majid Taie

Semmens, Caitlin

Semmens, Kathryn

Senalik, Christopher Adam

Senanayake, I. P.

Senanayake, Indishe

Senatore, Alfonso

Senay, Gebriel

Sengupta, Agniv

Senhadji-Navarro, Raouf 
Senyurek, Volkan Yusuf

Seo, Eunkyoung

Seo, Hwajeong

Seo, Ki-Weon

Seoane, Lucia

Sepasgozar, Samad

Seppänen, Jaakko

Sepúlveda Allende, Andrés

Sequeira, Ana

Sequeira, Jean

Serafino, Francesco

Şerban, Gheorghe

Serebryany, Andrey

Sergeev, Michael

Sergeeva, Maria

Sergieieva, Kateryna

Sergievskaya, I.

Sergio, Molina-Palacios

Šerić, Ljiljana

Serio, Carmine

Serkan Artagan, Salih

Serlenga, Vincenzo

Serra, Pere

Serra, Yolande

Serrano-Pérez, Antonio

Sertel, Elif

Servadio, Pieranna

Šestak, Ivana

Sestras,, Paul

Setiawati, Martiwi Diah

Severino Da Silva, Liliane

Sevil, J.

Séze, Geneviève M.

Sfetsos, Athanasios

Sfica, Lucian

Sguerso, Domenico

Sha, Zongyao

Shadaydeh, Maha

Shafiei Shiva, Javad

Shafique, Muhammad

Shah, Munam Ali

Shahab, Sina

Shahabfar, Alireza

Shah-Hosseini, Majid

Shahi, Kasra Rafiezadeh

Shahian Jahromi, Babak

Shahid, Imran

Shahidi, Reza

Shahri, Abbas

Shahtahmassebi, Amirreza

Shahzad, Imran
Shaik, Riyaaz Uddien

Shajahan, Sunoj

Shaker, George

Shamsafar, Faranak

Shamseldin, Tamer

Shamshiri, Redmond R.

Shang, Fang

Shang, Jiali

Shang, Rong

Shang, Shanshan

Shankar, Mohan

Shao, Guofan

Shao, Quanqin

Shao, Xi

Shao, Yang

Shaoxing, Mo

Sharifan, Hamidreza

Sharifi, Ayyoob

Sharifzadeh, Sara

Sharma, Abhishek

Sharma, Priyank J.

Sharma, Puneet

Sharma, Ram Krishan

Sharma, Ram P.

Sharma, Sonisa

Sharma, Suvash

Sharp, Ian

Sharpe, Shaun M.

Sharples, Ray

Shastry, Apoorva

Shaw, Harry

Shaw, Rajib

Shaw, Thomas E.

She, Bing

She, Jiangfeng

Sheekela, Baker-Yeboah

Shekaramiz, Mohammad

Shekhar, Ankit

Shelekhov, Alexander

Shelestov, Andrii

Shemer, Amir

Shen, Huanfeng

Shen, Hui

Shen, Hung Tao

Shen, Jianxiu

Shen, Jingwei

Shen, Kai

Shen, Ming

Shen, Qian

Shen, Wenjuan

Shen, Xiangjin 
Shen, Yonglin

Shen, Zhengkang

Shenghua, $\mathrm{Xu}$

Shestakov, Nikolay. V.

Shi, Changxing

Shi, Cheng

Shi, Chuang

Shi, Guangyao

Shi, Guoqiang

Shi, Hongling

Shi, Hongyin

Shi, Hoyeon

Shi, Hua

Shi, Kaifang

Shi, Kilin

Shi, Lei

Shi, Shuo

Shi, Wei

Shi, Yijun

Shi, Yun

Shi, Zhenwei

Shi, Zhongchao

Shi, Zhou

Shibata, Yuichiro

Shibusawa, Sakae

Shih, Peter

Shih, Tianyuan

Shihavuddin, A. S. M.

Shikhovtsev, Artem

Shikwambana, Lerato

Shimabukuro, Milton Hirokazu

Shimabukuro, Yosio

Shimada, Udai

Shimizu, Atsushi

Shimizu, Katsuto

Shin, Ju-Young

Shipman, Todd

Shiratsuchi, Luciano

Shirokov, Evgenii A.

Shirtliffe, Steve

Shirvani, Zeinab

Shiryaev, Anton G.

Shirzadi, Ataollah

Shiu, Yi-Shiang

Shoko, Cletah

Shokrolah Shirazi, Mohammad

Shrestha, Dhruba Pikha

Shrestha, Him L.

Shrestha, Maheswor

Shrestha, Narayan

Shrestha, Raju
Shrivastava, Sourabh

Shriver, Jay

Shuangguan, Donghui

Shugan, Igor

Shults, Roman

Shvetsov, Evgeny

Sibanda, Mbulisi

Sica, Francescopaolo

Sicard, Pierre

Siddiqui, Rehan

Sidorchuk, Aleksey

Sidorov, Denis

Siejka, Monika

Siems, Steven

Sieradzki, Rafał

Sifakis, Nicolas I.

Sileo, Maria

Silibello, Camillo

Sillero, Neftalí

Silow, Eugene

Silva Junior, Carlos Antonio Da

Silva Junior, Celso Henrique Leite

Silva, Adão

Silva, Bernardo

Silva, Carlos Alberto

Silva, Francisco Rodríguez Y.

Silva, Gabriela Ventura

Silva, João M. N.

Silva, Marx Leandro Naves

Silva, Valdson J.

Silva-Fuzzo, Daniela Fernanda

Silver, Micha

Silvestri, Sonia

Silwal, Abhisesh

Sim, Sunhui

Simioni, Emanuele

Simon, Edina

Simon, Francois-Xavier

Simone, Alessio

Simoneau, Alexandre

Simoni, Alessandro

Simoniello, Tiziana

Simonot, Lionel

Simonov, Nikolai

Simova, Petra

Simpson, Greg D.

Sinde-González, Izar

Singh, Aditya

Singh, Alka

Singh, Daljit

Singh, Deepak 
Singh, Jasreman

Singh, Minerva

Singh, Praveer

Singh, Rakesh Kumar

Singh, Ramesh P.

Singh, Sanjay

Singh, Saurabh

Singh, Shaktiman

Singh, Sushant

Sinica-Sinavskis, Juris

Siniscalchi, Sabato Marco

Sinkevich, Andrey A.

Sinnhuber, Björn-Martin

Sioris, Christopher E.

Sipelgas, Liis

Šipoš, Martin

Sirin, Andrey

Sirisena, T. A. Jeewanthi G.

Sirmaçek, Beril

Sirodoev, Igor

Sitokonstantinou, Vasileios

Sivanpillai, Ramesh

Sivarajan, Saravanan

Skakala, Jozef

Skarlatos, Dimitrios

Skilodimou, Hariklia D.

Sklute, Elizabeth

Skre, Oddvar

Škrinár, Andrej

Skripal, Anatoly

Skruch, Paweł

Skrzypkowski, Krzysztof

Skvareninova, Jana

Sladić, Dubravka

Slámová, Martina

Slavik, Martin

Sleewaegen, Jean Marie

Slezák, Michal

Sliwczynski, Lukasz

Slonecker, Terry

Smaczyński, Maciej

Small, Christopher

Smalley, Mark

Smart, Lindsey

Smedt, Isabelle De

Smekens, Jean-François

Śmiglak-Krajewska, Magdalena

Smirnova, Maria

Smith, Alex

Smith, Benjamin

Smith, Dave L.
Smith, Geoff

Smith, Kathryn

Smith, Nadia

Smith, Sharon

Smith, Stefan L.

Smith, Thomas M.

Smith-Flueck, Jo Anne

Smyshliev, Sergey

Smyth, Tim J.

Snellen, Mirjam

Snyder, Cory

Sobiech-Wolf, Jennifer

Sobieraj-Żłobińska, Anna

Sobkowiak, Leszek

Šobr, Miroslav

Sobue, Shinichi

Socas, Rafael

Socha, Jarosław

Sohel, Ferdous

Sohn, Chae-Bong

Sohn, Hong-Gyoo

Soilán, Mario

Søiland, Henrik

Soisuvarn, Seubson

Soja, Benedikt

Sojka, Mariusz

Sokol, Nicholas J.

Sokol, Zbyněk

Sola, Yolanda

Solano, Francesco

Solano-Correa, Yady Tatiana

Solaro, Giuseppe

Soldatenko, Sergei

Soldovieri, Francesco A.

Solla, Mercedes

Solomos, Stavros

Soltani, Fouzi

Soltani, Mohsen

Somkuti, Peter

Son, Pyo-Woong

Son, Seunghyun

Son, Youngsun

Sona, Giovanna

Song, Bonggeun

Song, Chang-Keun

Song, Cholho

Song, Gwo-Shyh

Song, Huihui

Song, Jinling

Song, Jiyun

Song, Joon 
Song, Meiping

Song, Qingjun

Song, Seungwon

Song, Shalei

Song, Wanjuan

Song, Wei

Song, Wenlong

Song, Xiaoqing

Song, Xiaoyu

Song, Zhen

Sonnemann, Till F.

Sonnessa, Alberico

Sonobe, Rei

Sood, Vishakha

Sorenson, Preston T.

Soria Garcia, Juan Miguel

Soria, Xavier

Soria-Ruiz, Jesus

Sośnica, Krzysztof

Sothe, Camile

Sotiriadis, Paul

Soukhovolsky, V. G.

Soulis, Konstantinos

Sousa, Ana Margarida Luís De

Sousa, António

Sousa, Daniel

Sousa, Joao Miguel

Sousa, Joaquim

Sousa, Maria João

Souza, Carlos

Souza, Eduardo G.

Souza, Richard

Souza, Rodolfo

Souza, Ronald B.

Sowden, Miles

Sowinska, Barbara

Sox, Leda

Sozzi, Marco

Spacher, Peter

Spaete, Lucas

Spandonidis, Christos

Spano, Antonia

Sparks, Aaron

Specht, Mariusz

Speight, Linda J.

Speranza, Eduardo Antonio

Sperdouli, Ilektra

Spiller, Dario

Spinetti, Claudia

Spiralski, Marcin

Spizzichino, Valeria
Splinter, Robert

Sportelli, Mino

Spracklen, Benedict

Sprintsin, Michael

Šprlák, Michal

Spyridon, Mavroulis

Sreckovic, Vladimir A.

Srinath, Indumathi

Srinivasan, Shruthi

Srivastava, Ankur

Srivastava, Prashant

Srivastava, Sanjeev

Sroka, Wojciech

Srokosz, Meric

Stabile, T. A.

Stach, Alfred

Stachlewska, Iwona

Stamatopoulos, Constantine A.

Stancalie, Gheorghe

Stancanelli, Laura

Stančerić, Ivica

Stancic, Ivo

Stanciu, Cristian

Stanič, Samo

Stanichny, Sergey

Staniek, Marcin

Stankevich, Sergey

Stanko, Davor

Stankovic, Zoran

Staples, Gordon

Starková, Lenka

Starosolski, Roman

Stateczny, Andrzej

Stateczny, Andrzej Eugeniusz

Stathopoulos, Nikolaos

Stathopoulou, Elisavet K.

Staver, Lorie W.

Stavrakoudis, Dimitris G.

Stavroula, Dimitriadou

Stavroulakis, Georgios G.

Stawowy, Marek

Stebel, Kerstin

Stefan, Markiewicz Jakub

Ştefan, Ovidiu

Stefańczyk, Maciej

Stefanescu, Razvan

Stefanidis, Stefanos

Steffen, Rebekka

Steffensen Schmidt, Louise

Stegmann, Patrick

Stegner, Alexandre 
Stehman, Stephen

Stein, Alfred

Steinberg, Andreas

Steinberg, Steven J.

Steinle, Peter

Stejskal, Jan

Stepanov, Oleg A.

Stepanova, Inna E.

Stepanyan, Karen

Stéphane, Kemkemian

Stępień, Michał

Stepinski, Tomasz F.

Sterckx, Sindy

Sternberg, Harald

Stewart, Christopher C.

Stewart, Robert R.

Sthel, Marcelo Silva

Stieglitz, Thomas C.

Stilianakis, Nikolaos I.

Stillinger, Timbo

Stiros, Stathis C.

Stjern, Camilla

Stoch, Tomasz

Stoffelen, Ad

Stoian, Andrei

Stojakovic, Vesna

Stojić, Andreja

Stonevicîus, Edvinas

Stopar, Bojan

Stopforth, Riaan

Storie, Joni

Strange, Andrew

Strasser, Sebastian

Stratoulias, Dimitris

Strelnikova, Dariia

Strelnikova, Irina

Stremme, Wolfgang

Strimbu, Bogdan M.

Strimbu, Vlad C.

Strobl, Peter

Strode, Sarah

Strohmeier, Michael

Strom, Alexander L.

Štroner, Martin

Stroppiana, Daniela

Strow, Larrabee

Strozzi, Tazio

Štular, Benjamin

Stupar, Aleksandra

Sturova, Izolda V.

Stütz, Peter
Štych, Přemek

Styers, Diane M.

$\mathrm{Su}$, Hongbo

$\mathrm{Su}, \mathrm{Hua}$

Su, Lihong

$\mathrm{Su}$, Nan-Jay

$\mathrm{Su}$, Tianning

$\mathrm{Su}$, Tung-Ching

$\mathrm{Su}$, Wei

$\mathrm{Su}$, Wen-Hao

$\mathrm{Su}$, Wenying

$\mathrm{Su}$, Xukun

$\mathrm{Su}$, Yanjun

$\mathrm{Su}, \mathrm{Yi}$

Su, Yuan-Fong

$\mathrm{Su}$, Zhong

Suarez Cadavid, Luz Angelica

Suarez, Alvaro

Suarez-Garcia, Andres

Subasi, Abdulhamit

Sublime, Jérémie

Suchocki, Czeslaw

Suciu, George

Sudmanns, Martin

Sui, Haigang

Suir, Glenn M.

Sujit, Sheeba Jenifer

Suk, Tomáš

Sukhov, Vladimir

Sukhova, Ekaterina

Suliman, Alaeldin

Sultana, Rebeka

Sumarsono, Alex

Sumbwanyambe, Mbuyu

Sümegi, Pál

Sun, Alexander

Sun, Baoqi

Sun, Bing

Sun, Chung-Hsun

Sun, Genyun

Sun, Guangcai

Sun, Haili

Sun, $\mathrm{Hu}$

Sun, Hua

Sun, Jinping

Sun, Junqiang

Sun, Kerry

Sun, Le

Sun, Lei

Sun, Leqiang

Sun, Liang 
Sun, Ling

Sun, Rui

Sun, Shangpeng

Sun, Shanlei

Sun, Shaobo

Sun, Shaohui

Sun, Weiwei

Sun, Wenbo

Sun, Yi

Sun, Yushan

Sun, Zhibin

Sundararaj, Vijayan

Sundberg, Robert

Sung, Andrew

Sung, Sangwoo

Sunny, Ali Imam

Suomalainen, Juha

Surovy, Peter

Susanto, R. Dwi

Susi, Melania

Suszanowicz, Dariusz

Suszyński, Robert

Sutherland, Ian

Suto, Hiroshi

Sužiedelytè-Visockienè, Jūratė

Suzuki, Kazuyoshi

Suzuki, Naoya

Suzuki, Taro

Svigkas, Nikos

Swaef, Tom De

Syed, Abbas Shah

Symeonaki, Eleni G.

Syrris, Vasileios

Szabo, Alexandre

Szabó, Szilárd

Szabolcsi, Róbert

Szabó-Takács, Beáta

Szatten, Dawid

Szatyłowicz, Jan

Száz, Dénes

Szczepanek, Robert

Szczepańska, Agnieszka

Szczepański, Emilian

Szczodrak, Malgorzata

Székely, Balázs

Szelag, Bartosz

Széles, Borbála

Szewczak, Kamil

Szewczyk, Roman

Sziebig, Gabor

Szilassi, Peter
Szkop, Artur

Szombara, Stanisław

Szopińska, Kinga

Szostak, Marta

Szporak-Wasilewska, Sylwia

Szturc, Jan

Szucs, Veronika

Szurgacz, Dawid

Szwedziak, Katarzyna

Szydłowski, Michał

Szymanowski, Mariusz

Szymczyk, Magdalena

Szypuła, Bartłomiej

Tabacu, Stefan

Tabaka, Przemyslaw

Tabatabaeenejad, Alireza

Taboada, Fernando González

Tack, Frederik

Tackett, Jason

Taddeo, Sophie

Tadesse, Haile K.

Tadić, Lidija

Tadini, Alessandro

Taghizadeh-Mehrjardi, R.

Taghizadeh-Mehrjardi, Ruhollah

Tagliaferro, Giulio

Taguchi, Kazunori

Tahami, Hoda

Tahjib-Ul-Arif, Md

Takala, Matias

Takashi, Machimura

Takebayashi, Hideki

Takewaka, Satoshi

Takoudjou Momo, Stéphane

Talchabhadel, Rocky

Talei, Amin

Talianu, Camelia

Talich, Milan

Talone, Marco

Talucci, Anna

Tamaddun, Kazi

Tamagnone, Mario

Tamari, Serge

Tamas, Eniko Anna

Tambouratzis, Tatiana

Tamisier, Thomas

Tamouridou, Afroditi A.

Tampucci, Marco

Tamulevičius, Gintautas

Tan, Jing

Tan, Junxiang 
Tan, Kun

Tan, Mou Leong

Tan, Xicheng

Tan, Yi

Tan, Yumin

Tan, Yuye

Tan, Zhenyu

Tan, Zhiqiang

Tanaka, Marcel Okamoto

Tanaka, Takashi

Tanaka, Yoshiyuki

Tananaev, Nikita

Tang, Guanglin

Tang, Guoqiang

Tang, Hong

Tang, Lin

Tang, Shiyang

Tang, Xiaojing

Tang, $\mathrm{Xu}$

Tang, $\mathrm{Yu}$

Tang, Yunchao

Tang, Zhiguang

Tangborn, Andrew

Tangdamrongsub, Natthachet

Tanguy, Maliko

Tanikawa, Tomonori

Tannant, Dwayne

Tao, Bo

Tao, Mingliang

Tao, Ran

Tao, Shengli

Tao, Xin

Tao, Xuanwen

Tao, Yu

Tapete, Deodato

Tapiador, Francisco J.

Tarabukina, L. D.

Taramasso, Angela Celeste

Taramelli, Andrea

Tarantino, Eufemia

Tarasenkov, M. V.

Taravat, Alireza

Tarolli, Paolo

Tarver, Hannah

Tashima, Tomoko

Taszakowski, Jarosław

Tateishi, Ryutaro

Tatsis, Konstantinos

Taubenböck, Hannes

Tavakkoli, Alireza

Tavani, Stefano
Tavares, Matheus Henrique

Tavares, Paulo Amador

Tavasci, Luca

Tavoularis, Nikolaos

Tayebi, Mahboobe

Taylor, Thomas E.

Tazioli, Alberto

Teatini, Pietro

Tebakari, Taichi

Tebaldini, Stefano

Ted, Gragson

Tefera, Gebrekidan Worku

Tegegne, Getachew

Teggi, Sergio

Tegos, Aristoteles

Tęgowski, Jaroslaw

Tehrani, Mahsa Jahandideh

Teixeira, Adunias Dos Santos

Teixeira, Danielle Cardozo Frasca

Teixeira, Marco Antonio

Teixeira, Marcos F. S.

Teixidó Ullod, M. Teresa

Tekavec, Jernej

Telfer, Matthew

Teli, Mohammad Nayeem

Tello, Marivi

Telnova, Natalia

Temnikov, Alexander

Tenace, Valerio

Tenedório, José António

Tenenbaum, David Elliot

Tenjo, Carolina

Tennina, Stefano

Teodoro, Ana Cláudia

Teodoro, Paulo Eduardo

Teppati Losè, Lorenzo

Terauchi, Genki

Terebes, Romulus

Terefenko, Paweł

Terenzio Gizzi, Fabrizio

Terra, Fabricio Da Silva

Terziev, Momchil

Tesche, Matthias

Teschl, Franz

Tesei, Giulio

Testoni, Nicola

Teunissen, Peter

Teza, Giordano

Tfwala, Samkele

Thakur, Balbhadra

Thakuri, Sudeep 
Theagarajan, Rajkumar

Theodoropoulos, Christos

Theodoulidis, Nikos

Thereon, Laetitia

Theuerkauf, Ethan J.

Thill, Jean-Claude

Thom, Christian

Thomas Ambadan, Jaison

Thomas, Brian F.

Thomas, Hugh

Thomas, Jeremy N.

Thomas, Pany

Thomas, Stefan

Thome, Kurt

Thompson, Amy

Thompson, Dan

Thompson, LuAnne

Thompson, Robert

Thomson, Bradley

Thomson, Jim

Thornton, Jeffrey A.

Thorwart, Martin

Thron, Christopher

Thurai, Merhala

Tian, Bo

Tian, Feng

Tian, Fuyou

Tian, Gui Yun

Tian, Haifeng

Tian, Jia

Tian, Jing

Tian, Tian

Tian, Wenxin

Tian, Ye

Ticehurst, Catherine

Tiebe, Carlo

Tien, Chung-Hao

Tiexi, Chen

Tijm-Reijmer, Carleen

Tijus, Charles

Tilley, Roger

Timár, Gábor

Timmermans, Joris

Timo, Balz

Timofeev, Vladimir

Tin, Pyke

Tiscornia, Guadalupe

Tisseyre, Bruno

Tiwari, Anuj

Tiwari, Surya Prakash

Tobak, Zalán
Tobie, Gabriel

Tobin, Kenneth J.

Toker, Onur

Tokola, Timo

Toledo, Daniel

Tölle, Merja Helena

Tolleson, Doug

Tolomei, Cristiano

Tomaselli, Valeria

Tomaszewska, Justyna

Tomaszewska, Monika Anna

Tomczak, Arkadiusz

Tomé, Ana

Tomei, Sonia

Tomelleri, Enrico

Tomljenovic, Ivan

Tommaselli, Antonio M. G.

Tommaso, Diomede

Tomppo, Erkki

Tonboe, Rasmus

Tonello, Kelly Cristina

Tong, Craig

Toniazzo, Alexander

Tonolo, Fabio Giulio

Topouzelis, Konstantinos

Topp, Simon

Torcasio, Rosa Claudia

Tordoni, Enrico

Toribio, Alan Miguel Velasquez

Tornow, Florian

Török, Ibolya

Torre, Carmelo Maria

Torregrosa, Alicia

Torres Sánchez, Jorge

Torres, Javier Martínez

Torres, Ricardo Da Silva

Torresan, Chiara

Torresani, Michele

Torres-Freyermuth, Alec

Torres-Perez, Juan

Torres-Sospedra, Joaquín

Torri, Marco

Tortini, Riccardo

Toscano, George

Toscano, Piero

Tosi, Daniele

Tosi, Luigi

Toth, Charles

Toth, Travis D.

Tóth, Zsolt

Tottrup, Christian 
Touhafi, Abdellah

Toure, Sory

Touzi, Ridha

Trabelsi, Rim

Trainor-Guitton, Whitney J.

Trakadas, Athena

Trammell, E. Jamie

Tran, Gia Khanh

Tran, $\mathrm{Ha}$

Tran, Hoang

Tran, Hoang Viet

Tran, Ngoc Nguyen

Trautz, Dieter

Tremblay, Denis

Trepal, Daniel

Trevisan, Rodrigo

Triantakonstantis, Dimitrios

Tribbia, Joseph

Trimble, Sarah

Tripcevich, Nicholas

Triplett, Colin

Tripolitsiotis, Achilleas

Triviño-Tarradas, Paula

Trizio, Ilaria

Trockel, Dale

Trockel, Josh

Trogu, Antonio

Trojanowska, Monika

Troncone, Antonello

Trout, Thomas

Trovato, Maria Rosa

Troyo-Diéguez, Enrique

Trpovski, Željen

Trung Nguyen, Huy

Truong Hoang, Vinh

Truong-Hong, Linh

Trzcinska, Karolina

Tsai, Chia-Lun

Tsai, Chi-Yi

Tsai, Fujung

Tsai, Hsiao-Ping

Tsai, HuiPing

Tsakanikas, Panagiotis

Tsakiridis, Nikolaos

Tsakirpaloglou, Nikolaos

Tsaramirsis, Georgios

Tsatsaris, Andreas

Tsekouras, George E.

Tsenova, Boryana

Tsiaras, Stefanos

Tsinos, Christos G.
Tsouros, Dimosthenis C.

Tsui, Brian Y.

Tsujino, Satoki

Tsuruta, Aki

Tsutsumida, Narumasa

Tsuyuki, Satoshi

Tsyrulnikov, Michael

$\mathrm{Tu}$, Chuan-Chi

$\mathrm{Tu}$, Kevin

$\mathrm{Tu}$, Rui

$\mathrm{Tu}$, Wei

$\mathrm{Tu}$, Yuhsuan

$\mathrm{Tu}$, Zhigang

Tucci, Biagio

Tucek, Jan

Tucu, Dumitru

Tulaikova, Tamara V.

Tuominen, Sakari

Turk, Goran

Tusset, Angelo Marcelo

Tutubalina, Olga

Tuxpan Vargas, Jose

Tuyishimire, Emmanuel

Tverberg, Vigdis

Tych, Wlodzimierz

Tychkov, Ivan I.

Typiak, Andrzej

Tyralis, Hristos

Tysiąc, Paweł

Tziachris, Panagiotis

Tziolas, Nikolaos

Tzouvaras, Marios

Uccheddu, Francesca

Uchiyama, Shoichiro

Udali, Alberto

Udina, Mireia

Uhl, Johannes $\mathrm{H}$.

Ujang, Uznir

Ukrainskiy, Pavel A.

Ulacha, Grzegorz

Ullah, Sami

Ullah, Waheed

Ullmann, Tobias

Ullo, Silvia

Ullo, Silvia Liberata

Ulpiani, Giulia

Um, Myoung-Jin

Umberto, Riccardi

Ungermann, Jorn

Ungureanu, Nicoleta

Uniyal, Bhumika 
Unnithan, Vikram

Uno, Itsushi

Upadhyaya, Shruti Ashok

Ur Rahman, Khalil

Ur Rehman, Muhammad Habib

Urban, Otmar

Urban, Rudolf

Urban, Thomas

Urbanski, Shawn

Urdea, Petru

Urquiza-Aguiar, Luis F.

Urquizo, Javier

Ursu, Adrian

Ury, Emily

Usery, E.

Uslaender, Thomas

Usowicz, Boguslaw

Uss, Mykhail

Ustalli, Nertjana

Ustinova, Evgeniya

Usui, Norihisa

Utsumi, Nobuyuki

Uudeberg, Kristi

Uuemaa, Evelyn

Uyeda, Kellie A.

Uzhinskiy, Alexander

Vabson, Viktor

Vacca, Giuseppina

Václavovic, Pavel

Vågen, Tor-Gunnar

Vahtmäe, Ele

Vaida, Ioana

Vain, Ants

Vaks, Vladimir

Valagussa, Andrea

Valcarce-Diñeiro, Rubén

Valcke, Roland

Valencia-Palomo, Guillermo

Valente, Antonio

Valente, Donatella

Valentin, Herbei Mihai

Valentin, Melissa M.

Valentini, Nico

Valeo, Caterina

Valerio, Emanuela

Väli, Germo

Valiante, Mario

Valiente, David

Valiente, Jose Antonio

Valipour, Mohammad

Valkaniotis, Sotirios
Vallebona, Chiara

Vallejo Perez, Moises Roberto

Vallet, Bruno

Vallianatos, Filippos

Valor, Enric

Valova, Iren

Valsesia, Diego

Van Arsdale, Roy

Van Baelen, Joel

Van De Giesen, Nick

Van De Wiel, Marco

Van Den Hoek, Jamon

Van Der Kwast, Hans

Van Der Kwast, Johannes

Van Der Merwe, Johannes Rossouw

Van Der Werff, Harald

Van Diedenhoven, Bastiaan

Van Doninck, Jasper

Van Gasselt, Stephan

Van Gent, Jeroen

Van Hamme, David

Van Leeuwen, Boudewijn

Van Malderen, Roeland

Van Noordwijk, Meine

Van Tricht, Kristof

Van Verre, Wouter

Van Wersch, Line

Van Wesemael, Bas

Van, Nguyen Thi Phuoc

Vandaele, Remy

Vandenberghe, Francois

Vanderhoof, Melanie

Vandermeulen, Ryan A.

Vanino, Silvia

Vanneschi, Claudio

Vannoppen, Astrid

Vardiambasis, Ioannis

Varela, Sebastian

Varenik, Alla

Varga, Domonkos

Varga, Orsolya

Vargas, Fabio

Vargas-Larreta, Benedicto

Vargin, Pavel Nikolaevich

Varlamov, Sergey M.

Varley, Nick

Varna, Inese

Varnagiryte-Kabasinskiene, Iveta

Várnai, Tamas

Varouchakis, Emmanouil A.

Varquez, Alvin Christopher Galang 
Vaščák, Ján

Vasconez, Juan. P

Vaseashta, Ashok

Vasil'ev, Denis

Vasilakos, Christos

Vasilyev, Roman

Vasques, Gustavo

Vassilios, Karakostas

Vatsanidou, Anna

Vaudour, Emmanuelle

Vavulin, Mikhail V.

Vaz, David

Vázquez, Francisco Gallego

Vázquez, Juan-Tomás

Vazquez, Patricia

Vazquez-Becerra, Guadalupe Esteban

Vázquez-Cuervo, Jorge

Vázquez-Jiménez, René

Vedel, Henrik

Vega, Miguel

Vega-Jorquera, Pedro

Veganzones, Miguel A.

Végvári, Zsolt S.

Velas, Martin

Velasco-Forero, Carlos

Velasquez, Nicolas

Velástegui-Montoya, Andrés

Velázquez, Ramiro

Vélez, Sergio

Veljanovski, Tatjana

Venafra, Sara

Venanzi, Rachele

Venegas, Pablo

Venetsanou, Panagiota

Venglár, Michal

Venter, Zander

Ventura, Daniele

Venturini, Virginia

Verbree, Edward

Verde, Natalia

Verde, Simona

Verdonck, Lieven

Verdun, Jérome

Veremeeva, Alexandra A.

Vergiev, Stoyan

Vergopolan, Noemi

Vergos, George

Verhagen, Henk Jan

Verhagen, Sandra

Verhegghen, Astrid

Verhoef, Anton
Verhoelst, Tijl

Verhoeven, Geert

Verhulst, Tobias

Verhulst, Tobias G. W.

Verma, Chaman

Vernier, Flavien

Verrelst, Jochem

Versaci, Mario

Verstraeten, Willem W

Vesely, Fosco M.

Vesnaver, Aldo

Vesnin, Artem M.

Vespremeanu-Stroe, Alfred

Vessio, Gennaro

Via, Stephen

Vian, André Luis

Viana, Helder

Vicario, Saverio

Vicente, Luiz Eduardo

Vicente-Guijalba, Fernando

Vichiensan, Varameth

Vickers, Hannah

Víctor, Molina García

Vida, György

Vieira Dutra, Luciano

Vieira, António

Vieira, Douglas Alexandre Gomes

Vieira, Vasco M. N. C. S.

Viennois, Gaëlle

Vignoli, Giulio

Vignudelli, Stefano

Vila, Daniel

Villadangos, Jesus

Villalón Turrubiates, Iván Esteban

Villalta, Ismael Vallejo

Villard, Ludovic

Villarreal, Miguel

Villarreal-Guerrero, Federico

Villarroel, Cristian

Villas-Boas, Paulino R.

Villegas, Dolors

Vinayak, Bhanage

Vincent, Charles Linwood

Vincent, Nicole

Vinci, Giacomo

Vindel, José María

Vinhas, Lubia

Vintila, Ruxandra

Vinukollu, Raghuveer K.

Virnodkar, Shyamal

Virolainen, Yana 
Virtanen, Juho-pekka

Virtanen, Timo H.

Visalli, Roberto

Vishwakarma, Shelly

Visockiene, Jurate

Vitagliano, Eleonora

Vitale, Marcello

Vitale, Sergio

Vitek, Stanislav

Vítková, Eva

Vitousek, Sean

Vitti, Alfonso

Vittuari, Luca

Vittucci, Cristina

Vizzari, Marco

Vlachogiannis, Diamando

Vladimir, Badenko

Voda, Mihai

Vodopivec, Martin

Vogel, Sören

Vogeler, Jody C.

Voiculescu, Mirela

Voinov, Sergey

Vojtek, Matej

Vojteková, Jana

Völksen, Christof

Von Appen, Wilken

Voogt, James

Voss, Kenneth

Votsi, Nefta-Eleftheria P.

Vozár, Luboš

Vozel, Benoit

Vretos, Nicholas

Vrieling, Anton

Vrochidis, Stefanos

$\mathrm{Vu}$, Tuong-Thuy

Vučić, Nikola

Vuelta, Arturo Colina

Vuillaume, Jean-Francois

Vujović, Igor

Vuletic, Dijana

Vysloužilová, Lenka

Vyzikas, Thomas

Wabiński, Jakub

Wada, Akiyoshi

Wadsworth, Richard A.

Waga, Hisatomo

Wagner, Frank

Wagner, Matthias P.

Wagner, Melissa

Wagner, Norman
Wahab, Ibrahim

Waheeb, Waddah

Waheed, Saddam Q.

Wahiduzzaman, Md

Waigl, Christine

Wakabayashi, Nobukazu

Walczykowski, Piotr

Walda, Jan

Waldner, Francois

Waldteufel, Philippe

Wałęga, Andrzej

Walker, John Withers

Walker, Nan

Walker, Victoria A.

Wallace, Cynthia

Wallner, Mario

Wallner, Stefan

Walo, Janusz

Walsh, Kerry

Walters, Linda

Walton, Gabriel

Wang, Andong

Wang, Caixia

Wang, Caixin

Wang, Changcheng

Wang, Cheng

Wang, Chenghao

Wang, Chi-Kuei

Wang, Chuyuan Carter

Wang, Cuizhen

Wang, Dagang

Wang, Dalei

Wang, Dezhi

Wang, Difeng

Wang, Dingkang

Wang, Dingyi

Wang, Dongdong

Wang, Dongxiang

Wang, Dongyi

Wang, Fu-Kang

Wang, Fuyao

Wang, Guangxing

Wang, Guifen

Wang, Guoping

Wang, Guoquan

Wang, Haibo

Wang, Haihong

Wang, Hao

Wang, He

Wang, He-sheg

Wang, Hongquan 
Wang, Hsueh-Ching

Wang, $\mathrm{Hu}$

Wang, Hui

Wang, Huimin

Wang, Jia

Wang, Jianmin

Wang, Jianxin

Wang, Jie

Wang, Jingxia

Wang, Jingyu

Wang, Jingzhe

Wang, Juanle

Wang, Jun

Wang, Junbang

Wang, Jungang

Wang, Kai

Wang, Kuo-Lung

Wang, Lanhui

Wang, Lei

Wang, Long

Wang, Longlong

Wang, Lunche

Wang, Menghua

Wang, Muyang

Wang, Nan

Wang, Ningbo

Wang, Pei

Wang, Peng

Wang, Ping

Wang, Ran

Wang, Ruci

Wang, Shaohua

Wang, Sheng

Wang, Shengqiang

Wang, Shugong

Wang, Szu-Hua

Wang, Tao

Wang, Taoyang

Wang, Teng

Wang, Tianlin

Wang, Tiejun

Wang, Wei

Wang, Wenhui

Wang, Wenjuan

Wang, Wenqing

Wang, Wenyuan

Wang, Xian

Wang, Xianpeng

Wang, Xianwei

Wang, Xiaoming

Wang, Xuantong
Wang, Xuejia

Wang, Xuezhi

Wang, Xufeng

Wang, Yafei

Wang, Yanping

Wang, Yawen

Wang, Yetang

Wang, Yexin

Wang, Yi

Wang, Yongbo

Wang, Yu

Wang, Yuanbing

Wang, Yuebin

Wang, Yuhao

Wang, Yun-Tao

Wang, Zengmao

Wang, Zhe

Wang, Zhen

Wang, Zhengtao

Wang, Zhenzhan

Wang, Zhixiong

Wang, Zhongjing

Wang, Zhongliang

Wang, Zhuosen

Wang, Zongming

Wapenaar, Cornelis P. A.

Waqas, Prince

Warland, Jon S.

Warnock, April

Warren, Mark

Warren, Stacy

Wasserzier, Christoph

Watanabe, Fernanda

Watanabe, Manabu

Watanabe, Shuntaro

Watanabe, Takuma

Watkins, James

Watson, Des

Watson, Simon

Watts, Simon

Wawruch, Ryszard

Wąż, Mariusz

Webb, Geoffrey I.

Webb, Ryan

Weber, Christiane

Weber, Helga

Weber, Iris

Weber, Keith

Weber, Konradin

Weber, Maridee

Weber, Markus 
Weber, Robert

Wehbe, Youssef

Wei, Guohua

Wei, Haoran

Wei, Jing

Wei, Shanshan

Wei, Shunjun

Wei, Xinyuan

Wei, Zhangping

Weichbroth, Paweł

Weigel, Robert

Weissgerber, Flora

Wells, Kelley

Wempen, Jessica M.

Wen, Haijia

Wen, Jianguang

Wen, Li

Wendt, Anja

Weng, Duojie

Weng, Fuzhong

Wenzel, Helmut

Werhahn, Olav

Werner, Małgorzata

Wessel, Birgit

Wester, David B.

Westerhoff, Rogier

Westerholt, René

Westley, Kieran

Wetterlind, Johanna

Wezka, Kinga

Wheate, Roger

Wheater, Howard

Whelley, Patrick

White, H. Peter

White, Raechel

White, Scott

Wick, Gary A.

Widjaja Putra, Bayu Taruna

Widodo, Joko

Widyaningrum, Elyta

Wiederhold, Helga

Wiehle, Stefan

Wiekenkamp, Inge

Wielgosz, Pawel

Wierzbicki, Damian

Wiesner, Susanne

Wijayarathne, Dayal

Wijesingha, Jayan

Wijewardane, Nuwan

Wijnhoven, Rob

Wiktor, Tracz
Wiley, Robert

Wilhelm, Thorsten

Wilkes, Phil

Wilkes, Thomas C.

Wilkinson, Ben

Wilkowski, Artur

Will, Kochtitzky

Willemsen, Thomas

Willén, Erik

Williams, David P.

Williams, Gustavious Paul

Williams, Roger A.

Williams, Simon

Willmes, Sacha

Wilsey, Brian

Wilson, Barry T.

Wilson, Kyle

Wilson, Truman

Wimberly, Mike

Winfrey, Brandon K.

Wing, Robin

Winkler, Hartmut

Wińska, Małgorzata

Wirion, Charlotte

Wise, Nicholas

Wiśniewski, Łukasz

Wiśniewski, Szymon

Witkowski, Wojciech

Witthuhn, Jonas

Woche, Susanne K.

Wohler, Christian

Wohlfarth, Kay

Wojciechowski, Tomasz

Wójcik-Leń, Justyna

Wojnowski, Wojciech

Wojtczak, Erwin

Wolters, Erwin

Won, Jong-Hoon

Wong, YingQi

Wood, David

Wood, Melissa

Woolliams, Emma

Wrenger, Burkhard

Wrona, Paweł

Wrzesiński, Dariusz

$\mathrm{Wu}$, Aisheng

$\mathrm{Wu}$, Bangyu

Wu, Baosheng

$\mathrm{Wu}, \mathrm{Bin}$

Wu, Chaofan

Wu, Chih-Da 
Wu, Dien

$\mathrm{Wu}$, Donghai

$\mathrm{Wu}$, Dong-Hong

$\mathrm{Wu}$, Falin

$\mathrm{Wu}$, Fan

Wu, Hangbin

$\mathrm{Wu}$, Hua

$\mathrm{Wu}$, Jian-Hong

$\mathrm{Wu}$, Jianwei

Wu, Ke

Wu, Lianhui

$\mathrm{Wu}$, Lin

$\mathrm{Wu}$, Minquan

Wu, Mousong

$\mathrm{Wu}$, Penghai

Wu, Qinggang

Wu, Qisong

$\mathrm{Wu}$, Shangrong

$\mathrm{Wu}$, Shengbiao

Wu, Shuang-Ye

$\mathrm{Wu}$, Taixia

$\mathrm{Wu}$, Ting

$\mathrm{Wu}$, Tonghua

$\mathrm{Wu}$, Wan

$\mathrm{Wu}$, Wenjin

Wu, Xiaocui

Wu, Xiaoliang

Wu, Xuerui

Wu, Yiping

Wu, Zebin

$\mathrm{Wu}, \mathrm{Zhu}$

Wyatt, Briana

Wysocki, Douglas

Wysocki, Marian

Wyszkowska, Patrycja

Xanthopoulos, Georgios

Xavier, António M. S.

Xavier, Dérobert

Xavier-de-Souza, Samuel

Xi, Baike

Xi, Hongyan

Xi, Xiaohuan

Xia, Haoming

Xia, Linyuan

Xia, Min

Xia, Renbo

Xia, Shaobo

Xia, Xiang'ao

Xiang, Deliang

Xiao, Chiwei

Xiao, Feng
Xiao, Guorui

Xiao, Qiyang

Xiao, Ruya

Xiao, Xiongwu

Xiao, Xiongxin

Xiao, Yang

Xiao, Zhifeng

Xiaomeng, Geng

Xiaoying, Ouyang

Xie, Donghui

Xie, Hong

Xie, Hongjie

Xie, Huan

Xie, Jing

Xie, Lingling

Xie, Qiaoyun

Xie, Qinghua

Xie, Tao

Xie, Xuefeng

Xie, Yichun

Ximenes, Arimatéa

Xin, $\mathrm{Wu}$

Xin, Xiaozhou

Xing, Hanfa

Xing, Jin

Xing, Minfeng

Xing, Xuemin

Xing, Yanqiu

Xiong, Chuan

Xiong, Fengchao

Xiong, Jiping

Xiong, Kai

Xiong, Lin

Xiong, Liyang

Xiong, Pan

Xiong, Siting

Xiong, Wen

Xiong, Yongzhu

Xiucheng, Yang

Xofis, Panteleimon

$\mathrm{Xu}$, Baodong

$\mathrm{Xu}$, Bing

$\mathrm{Xu}$, Caijun

$\mathrm{Xu}$, Chong

$\mathrm{Xu}$, Congan

Xu, Erqi

$\mathrm{Xu}$, Guanjun

$\mathrm{Xu}$, Guirong

$\mathrm{Xu}$, Huaping

Xu, Hui

Xu, Jia 


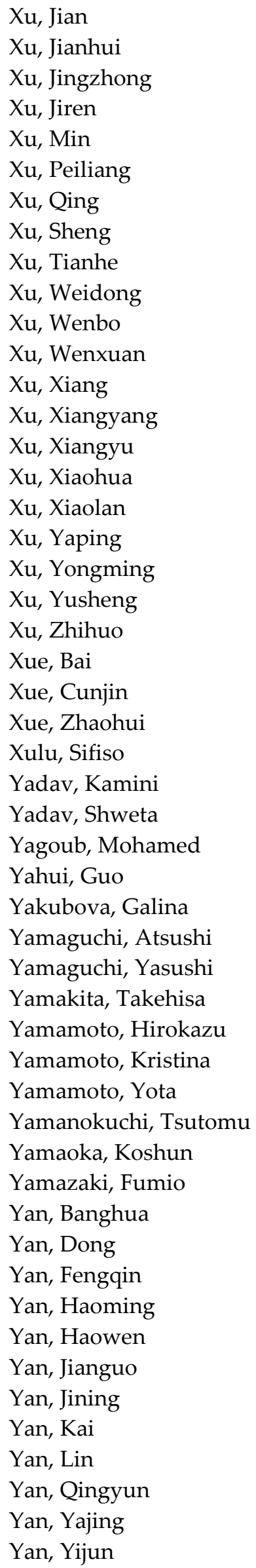

Yang, Bo

Yang, Ce

Yang, Chunxue

Yang, Ci Jian

Yang, Di

Yang, Dingtian

Yang, Dongkai

Yang, Fanlin

Yang, Fei

Yang, Feifei

Yang, Haiping

Yang, Hanbo

Yang, $\mathrm{Hu}$

Yang, Jason

Yang, Jian

Yang, Jianwei

Yang, Jingxiang

Yang, Jinn-Min

Yang, Jinxiang

Yang, Jiuchun

Yang, Jong-Ryul

Yang, Jun

Yang, Jungang

Yang, Kailun

Yang, Kang

Yang, Liping

Yang, Meijian

Yang, Meng

Yang, Ming-Der

Yang, Peiqi

Yang, Qi

Yang, Shih-Sian

Yang, Wanneng

Yang, Wei

Yang, Wenze

Yang, Xiaolei

Yang, Xiaoying

Yang, Xihua

Yang, Xiucheng

Yang, Xue

Yang, Yang

Yang, Yanjun

Yang, Yongzeng

Yang, Yuanjian

Yang, Yuekui

Yang, Yunping

Yang, Zefa

Yang, Zhiqiang

Yannopoulos, Stavros

Yanxiong, Liu

Yao, Dongdong 


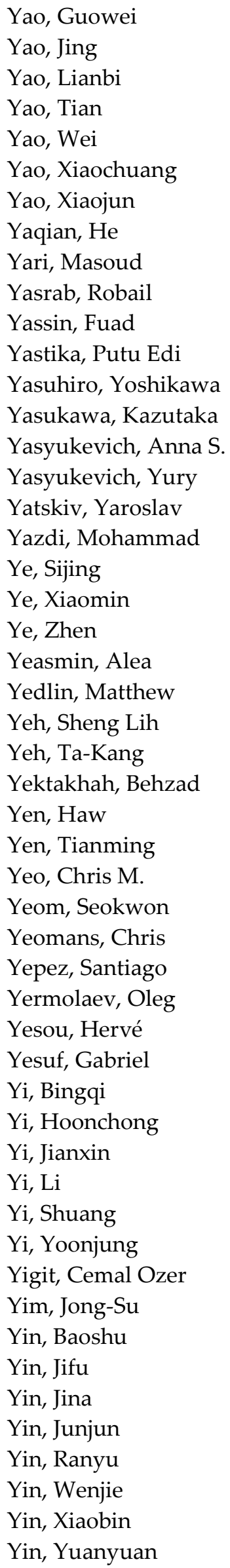

Yin, Zhenliang

Ying, Qing

Ying, Shen

Yitayew, Temesgen Gebrie

Ylänne, Henni

Yokota, Sho

Yonel, Bariscan

Yong, Wang

Yoo, Cheolhee

Yoo, Jaeseon

Yoo, Jung-Moon

Yoon, Hyosang

Yoon, Hyungchul

Yoon, Joo-eun

Yordanov, Vasil

Yoshida, Takahiro

Yoshikawa, Takashi

Yoshioka, Akira

You, Cheol-Hwan

You, Lingyun

You, Yanan

Young, Derek

Young, Ian

Young, Mary

Young, Ruth

Young, Tun Jan

Yousefi, Bardia

Yousefi, Mahdi

Yousefzadeh, Mohsen

Youssef, Ahmed M.

Yrttimaa, Tuomas

$\mathrm{Yu}$, Anxi

$\mathrm{Yu}$, Chen

$\mathrm{Yu}$, Chih-Chang

Yu, Fangjie

$\mathrm{Yu}$, Gongliang

Yu, Hanwen

Yu, Haoyang

Yu, Huapeng

Yu, Jiayong

Yu, Kefu

Yu, Tianqi

Yu, Ting-To

Yu, Xiaolei

Yu, Xuelian

Yu, Zhaowu

Yu, Zhiyuan

Yuan, Cui

Yuan, Fenghui

Yuan, Peng

Yuan, Qiangqiang 
Yuan, Titus

Yuan, Wei

Yuan, Wenan

Yuan, Xinzhe

Yuan, Xiuliang

Yuan, Yunbin

Yuchechen, Adrián

Yudin, Dmitry

Yue, Jibo

Yue, Linwei

Yue, Lishengsa

Yue, Shichao

Yun, Ting

Yun, Youngsun

Yurganov, Leonid

Yurovsky, Yury

Yuste Delgado, Antonio Jesús

Yuzugullu, Onur

Yüzügüllü, Onur

Yvinec, Yann

Zabalza, Jaime

Zabret, Katarina

Zabulis, Xenophon

Zaccheo, T. Scott

Zaccone, Raphael

Zadorozhny, Alexander M.

Zaeen, Ahmed

Zafra, Carlos

Zagajewski, Bogdan

Zagar, Bernhard

Zagar, Dusan

Zagar, Mark

Zahiri, Zohreh

Zaimes, George

Zaina, Federico

Zaitouny, Ayham

Zakharov, Alexander F.

Zakharov, Alexander I.

Zakharov, Igor

Zakharova, Natalia I.

Zakinyan, Robert

Zakšek, Klemen

Zald, Harold

Zalewski, Paweł

Zalidis, George

Zambrano Bigiarini, Francisco

Zamfir, Daniela

Zamfir, Rares Halbac-Cotoara

Zampa, Luigi

Zamparelli, Virginia

Zan, Xuli
Zanetti, Massimo

Zang, Hengchang

Zang, Xiaoqin

Zangeneh, Pouya

Zanimonskiy, Yevgen M.

Zanutta, Antonio

Zaplana, Isiah

Zappa, Luca

Zaramella, Mattia

Zarayskaya, Yulia

Zare, Azimeh

Zareapoor, Masoumeh

Zaron, Ed

Żarski, Jacek

Zarzalejo, Luis Fernando

Zarzar, Christopher

Zarzo-Arias, Alejandra

Zasadil, Petr

Zatelli, Paolo

Zaugg, Evan

Zavagli, Massimo

Zavorotny, Valéry

Zawadzka, Joanna E.

Zawadzki, Jarosław

Zawadzki, Lionel

Zazo, Santiago

Zdorovennova, Galina

Zdravevski, Eftim

Zdunek, Rafal

Zec, Josko

Zeeshan Ali, Muhammad

Zeferino, Cesar Albenes

Zehtabian, Amin

Zeid, Nasser Abu

Zelazowski, Przemyslaw

Zelensky, Nikita

Zemtsov, Valeriy

Zen, Simone

Zeng, Jiangyuan

Zeng, Qiang

Zeng, Runqiang

Zeng, Tieyong

Zeng, Wei Sheng

Zeng, Yelu

Zeng, Zhaoliang

Zeng, Zhe

Ženka, Jan

Zeraatpisheh, Mojtaba

Zeri, Marcelo

Zeroual, Ayoub

Zewdie, Worku 
Zha, Yuanyuan

Zhai, Deli

Zhai, Wei

Zhamsueva, Galina S.

Zhan, Yizhe

Zhang, Anlu

Zhang, Banglin

Zhang, Baocheng

Zhang, Bin

Zhang, Bohai

Zhang, Chen

Zhang, Chengye

Zhang, Chu

Zhang, Chuanrong

Zhang, Chunhua

Zhang, Dayi

Zhang, Enze

Zhang, Eric J.

Zhang, Fan

Zhang, Fangzhao

Zhang, Frank

Zhang, Guoqing

Zhang, Haifeng

Zhang, Hankui

Zhang, Hao

Zhang, Haopeng

Zhang, Hong

Zhang, Hongsheng

Zhang, Huanxin

Zhang, Jiahua

Zhang, Jian

Zhang, Jiashu

Zhang, Jiaxing

Zhang, Jiaying

Zhang, Junzhe

Zhang, Ke

Zhang, Lamei

Zhang, Lefei

Zhang, Lei

Zhang, Leo

Zhang, Liangpei

Zhang, Lifu

Zhang, Lin

Zhang, Ling

Zhang, Lu

Zhang, Miao

Zhang, Min

Zhang, Minwei

Zhang, Pengfei

Zhang, Qingjun

Zhang, Qingling
Zhang, Qingyuan

Zhang, Qiuzhao

Zhang, Qun

Zhang, Rui

Zhang, Ruiqian

Zhang, Runsen

Zhang, Shaojie

Zhang, Shengkai

Zhang, Shuai

Zhang, Shuangcheng

Zhang, Shuimei

Zhang, Su

Zhang, Tianwen

Zhang, Tianxian

Zhang, Tingbin

Zhang, Tong

Zhang, Wanchang

Zhang, Weixing

Zhang, Wen

Zhang, Wenjuan

Zhang, Wu

Zhang, Xi

Zhang, Xianfeng

Zhang, Xiang

Zhang, Xiaohu

Zhang, Xiaoli

Zhang, Xiaoling

Zhang, Xingyi

Zhang, Xiongqing

Zhang, Xuebo

Zhang, Xuedong

Zhang, Xun

Zhang, Yakun

Zhang, Yali

Zhang, Yanchao

Zhang, Yi

Zhang, Yihang

Zhang, Yin

Zhang, Ying

Zhang, Yize

Zhang, Yizhuo

Zhang, Yong

Zhang, Yonghong

Zhang, Yuanchong

Zhang, Yuanzhi

Zhang, Yueting

Zhang, Yun

Zhang, Yunsheng

Zhang, Yuxiang

Zhang, Yuzheng

Zhang, Zenghui 
Zhang, Zeyu

Zhang, ZhaoMing

Zhang, Zhenchao

Zhang, Zhengguang

Zhang, Zhenxin

Zhao, Chaofang

Zhao, Chaoying

Zhao, Chuanfeng

Zhao, Chuihui

Zhao, Chunhong

Zhao, Enyu

Zhao, Fang

Zhao, Feng

Zhao, Hengqian

Zhao, Jiangsan

Zhao, Jiannan

Zhao, Jinling

Zhao, Meng

Zhao, $\mathrm{Na}$

Zhao, Qian

Zhao, Qinzhi

Zhao, Sihao

Zhao, Tianjie

Zhao, Tianliang

Zhao, Tiebiao

Zhao, Wei

Zhao, Wenzhi

Zhao, Xi

Zhao, Xiang

Zhao, Xiaoyi

Zhao, Xinyue

Zhao, Yan

Zhao, Yifan

Zhao, Yixin

Zhao, Yuefeng

Zhao, Zhongxiang

Zharkov, Maksim

Zhdanov, Michael S.

Zhen, Zhen

Zheng, Gang

Zheng, Hongxing

Zheng, Jiafeng

Zheng, Jibin

Zheng, Juepeng

Zheng, Minghua

Zheng, Qiming

Zheng, Qinghe

Zheng, Quanan

Zheng, Senlin

Zheng, Shuanning

Zheng, Ting
Zheng, Xingming

Zheng, Xinqi

Zheng, Yuhui

Zheng, Zhoutao

Zheng, Zhubin

Zheng, Zihao

Zhenyu, Fang

Zhi, Huang

Zhiyun, Jiang

Zhogolev, Arseniy

Zhong, Bo

Zhong, Detang

Zhong, Jiahao

Zhong, Xing

Zhong, Yulong

Zhou, Chen

Zhou, Chengwei

Zhou, Decheng

Zhou, Feng

Zhou, Guangsheng

Zhou, Guiyun

Zhou, Hao

Zhou, Hongmin

Zhou, Ji

Zhou, Jie

Zhou, Jing

Zhou, Lianjie

Zhou, Lifan

Zhou, Meng

Zhou, Minqiang

Zhou, Peiyuan

Zhou, Qiang

Zhou, Shanshi

Zhou, Song

Zhou, Tian

Zhou, Wen

Zhou, Xiong

Zhou, Xiran

Zhou, Yonghong

Zhou, Yuke

Zhou, Zheng-Shu

Zhou, Zhiqiang

Zhu, Dan

Zhu, Gaohua

Zhu, Jianjun

Zhu, Jinshan

Zhu, Liping

Zhu, Liujun

Zhu, Qing

Zhu, Rui

Zhu, Shuang 
Zhu, Weining

Zhu, Wenbin

Zhu, Wenquan

Zhu, Xiaolin

Zhu, Xiufang

Zhu, Xuan

Zhu, Xu-Dong

Zhu, Zaichun

Zhu, Zhengliang

Zhu, Zhongben

Zhuang, Wei

Zhuang, Yizhou

Zhuge, Xiaoyong

Zhuravlev, Andrey

Zhuravleva, T. B.

Zhurbas, Victor M.

Zia, Muhammad Yousuf Irfan

Ziaja, Wieslaw

Zibordi, Giuseppe

Žibrat, Uroš

Zieger, Silvia E.

Zielewska-Büttner, Katarzyna

Zielhofer, Christoph

Zikidis, Konstantinos C.

Zima, Krzysztof

Zinn, Yuri L.

Ziolkowski, Patryk

Ziskin, Daniel

Zivcak, Marek

Žížala, Daniel

Zlatev, Zlatin

Zlotnicki, Victor

Zmarz, Anna

Zoccatelli, Davide
Zodiatis, George

Zoeke, Dominik

Zoffoli, Laura

Żokowski, Mariusz

Zollini, Sara

Zomeni, Zomenia

Zoppi, Corrado

Zorzi, Mattia

Zotta, Ruxandra-Maria

Zotti, Georg

Zou, Bin

Zou, Defu

Zou, Lilong

Zou, Yanhong

Zou, Zheguang

Zou, Zhenhua

Zovko, Monika

Zribi, Mehrez

Zrinjski, Mladen

Źróbek, Ryszard

Zubelzu Minguez, Sergio

Zubov, Vladimir

Zuleta, Gustavo

Zullo, Francesco

Zúñiga, Emmanuel

Župan, Robert

Zupanski, Dǔsanka

Zurqani, Hamdi

Zus, Florian

Zweig, Christa L.

Zwoliński, Zbigniew

Zygouri, V.

Zyśk, Janusz 